Aus der Klinik für Neurochirurgie

(Prof. Dr. med. V. Rohde)

der Medizinischen Fakultät der Universität Göttingen

\title{
Etablierung eines Grading-Systems zur Beurteilung des Schweregrades experimenteller
}

\section{Subarachnoidalblutungen im Rattenmodell}

\author{
INAUGURAL-DISSERTATION \\ zur Erlangung des Doktorgrades \\ der Medizinischen Fakultät der \\ Georg-August-Universität zu Göttingen
}

vorgelegt von

Vesna Malinova

aus

Stip, Mazedonien

Göttingen 2018 
Dekan:

\section{Betreuungsausschuss}

Betreuerin:

Ko-Betreuer:

\section{Prüfungskommission}

Referent/in

Ko-Referent/in:

Drittreferent/in:

Datum der mündlichen Prüfung:
Prof. Dr. rer. nat. H.K. Kroemer

Prof. Dr. med. D. Mielke

PD Dr. med. M. N. Psychogios

Prof. Dr. med. Dorothee Mielke

PD Dr. med. Marios-Nikos Psychogios

Prof. Dr. Thomas Meyer 
Hiermit erkläre ich, die Dissertation mit dem Titel "Etablierung eines Grading-Systems zur Beurteilung des Schweregrades experimenteller Subarachnoidalblutungen im Rattenmodell" eigenständig angefertigt und keine anderen als die von mir angegebenen Quellen und Hilfsmittel verwendet zu haben.

Göttingen, den

(Unterschrift) 


\section{Inhaltsverzeichnis}

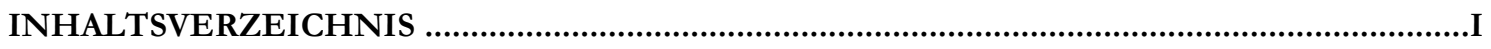

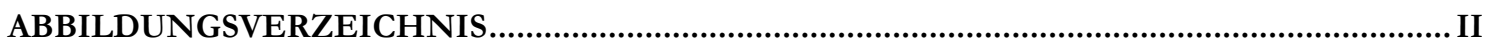

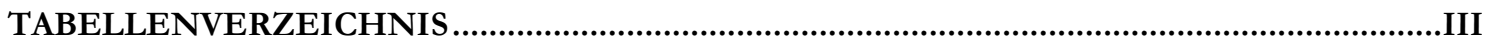

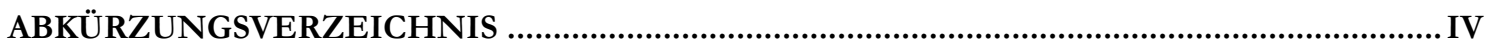

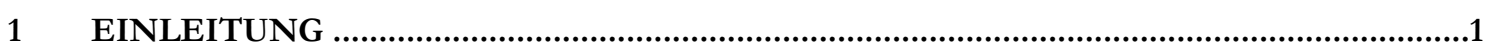

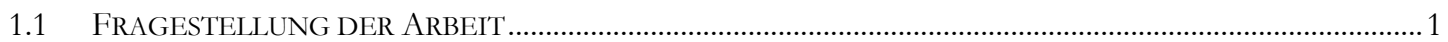

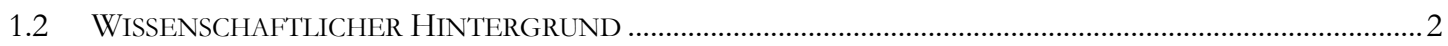

1.2.1 Subarachnoidalblutung (Definition, Epidemiologie und Ätiologie) ..............................................2

1.2.2 Klinik, Diagnostik und Klassifikation der SAB ................................................................................ 8

1.2.3 Frühe Hirnschädigung (early brain injury) nach einer SAB …........................................................12

1.2.4 Verzögerte zerebrale Ischämie (delayed cerebral ischemia) nach einer SAB ....................................12

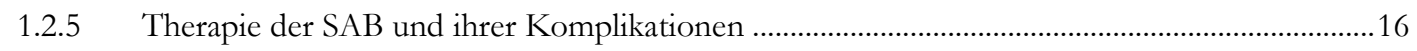

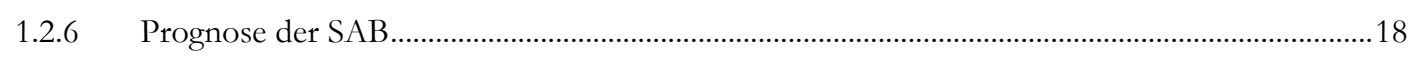

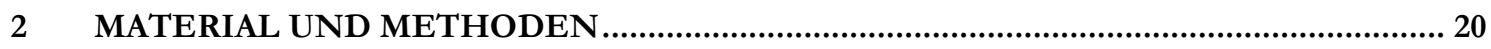

2.1 TIERMODELL FÜR DIE EXPERIMENTELLE SUBARACHNOIDALBLUTUNG................................................20

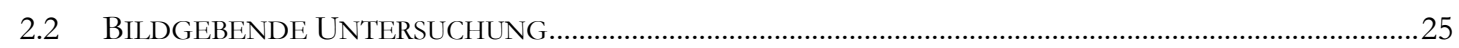

2.2.1 MRT-Untersuchungen ...........................................................................................................25

2.3 HistologisCHE UNTERSUCHUNGEN ……......................................................................................25

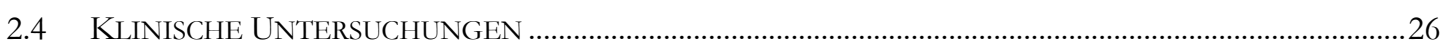

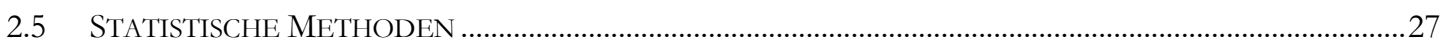

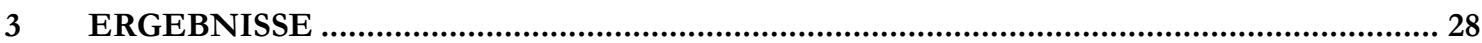

3.1 INDUKTION DER SAB MIT DEM DOUBLE HEMORRHAGE MODEL …....................................................28

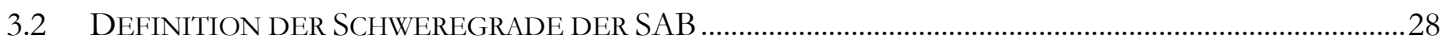

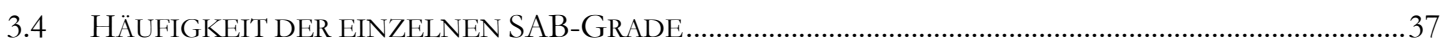

3.5 KLINISCHER UND NEUROLOGISCHER STATUS DER RATTEN ……...........................................................3

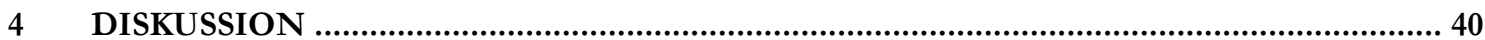

4.1 DISKUSSION DER KLASSIFIKATIONSSYSTEME DER BLUTUNG …….....................................................42

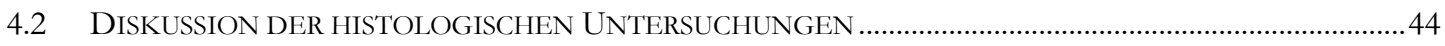

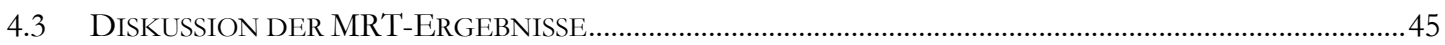

4.4 LIMITATION UND STÄRKE DER ARBEIT....................................................................................................46

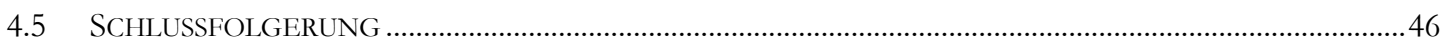

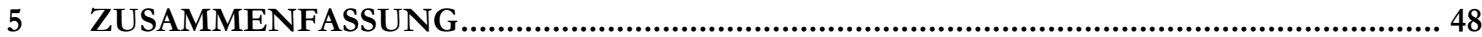

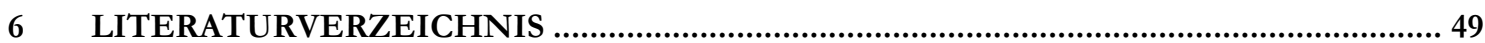




\section{Abbildungsverzeichnis}

Abbildung 1: Basale Cisternen und Hirnventrikel ..............................................................................................2

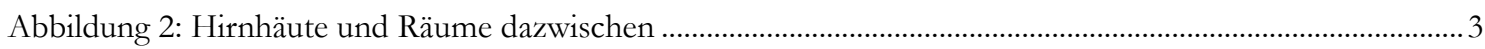

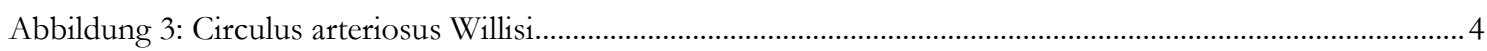

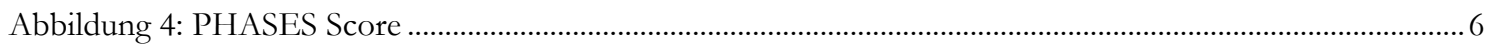

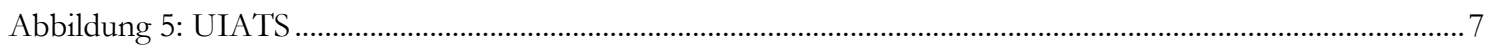

Abbildung 6: Aneurysmatische Subarachnoidalblutung (CCT und CTA) ………............................................... 9

Abbildung 7: DSA mit einem Aneurysma der A. cerebri media rechts .................................................................

Abbildung 8: Operationssitus unter Mikroskop vor und nach Clipping eines Aneurysma .................................17

Abbildung 9: Darstellung eines Basilariskopfaneurysma vor und nach Coiling...................................................18

Abbildung 10: Einspannen des Kopfes im stereotaktischen Rahmen ...................................................................22

Abbildung 11: Darstellung der Membrana atlantooccipitalis ............................................................................23

Abbildung 12: Portable Animal Intensive Care Unit .............................................................................................23

Abbildung 13: Experimentelle SAB nach dem doppelten Injektionsmodell bei der Ratte ...................................24

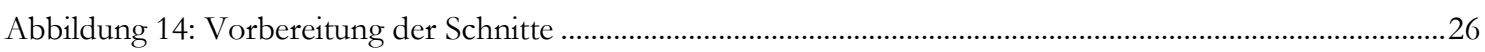

Abbildung 15: Grad-1-Blutung (Darstellung des Ventrikels mit Plexus choroideus) .........................................22

Abbildung 16: Grad-1-Blutung (Darstellung eines Anteils der linken Hemisphäre) ..........................................229

Abbildung 17: Grad-1-Blutung (Darstellung eines Anteils der Hirnoberfläche) .................................................29

Abbildung 18: Grad-2-Blutung (Darstellung der Hirnoberfläche mit sichtbarer Blutung) ....................................30

Abbildung 19: Grad-2-Blutung (Stärkere Vergrößerung mit Darstellung des Blutes) ...........................................3 30

Abbildung 20: Grad-2-Blutung (Darstellung des Ventikels ohne sichtbare Blutung) ...........................................31

Abbildung 21: Grad-2-Blutung (Stärkere Vergrößerung mit Darstellung des Plexus choroideus).......................31

Abbildung 22: Grad-2-Blutung (Ausgeprägte diffus verteilte Blutung an der Hirnoberfläche) ...........................32

Abbildung 23: Grad-2-Blutung (Stärkere Vergrößerung mit Darstellung der Blutverteilung) .............................32

Abbildung 24: Grad-2-Blutung (Stärkere Vergrößerung mit Darstellung der Blutmenge) ...................................33

Abbildung 25: Grad-3-Blutung (Darstellung eines lokalen Blutclots im Subarachnoidalraum) ..........................33

Abbildung 26: Grad-3-Blutung (Stärkere Vergrößerung mit Darstellung der lokalen Blutung) ...........................33

Abbildung 27: Grad-3-Blutung (Ausgeprägte lokale Blutung im Subarachnoidalraum) ........................................34

Abbildung 28: Grad-3-Blutung (Stärkere Vergrößerung mit Fokus auf die Blutung)...........................................34

Abbildung 29: Grad-4-Blutung (Ventrikel mit ausgeprägter intraventrikulärer Blutung)......................................35

Abbildung 30: Grad-4-Blutung (Stärkere Vergrößerung mit Fokus auf die Ventrikelblutung).............................35

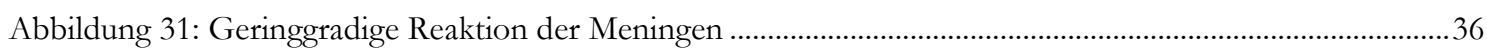

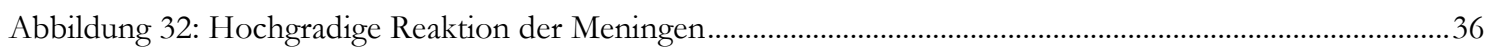

Abbildung 33: Verteilung der einzelnen SAB-Grade in der SAB-Gruppe ...............................................................37

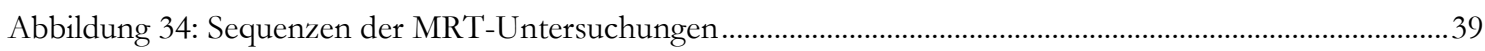




\section{Tabellenverzeichnis}

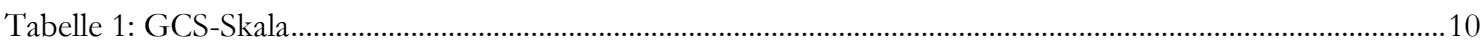

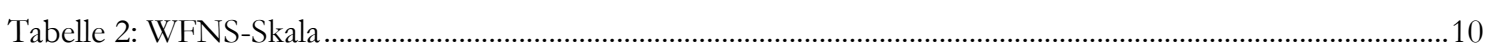

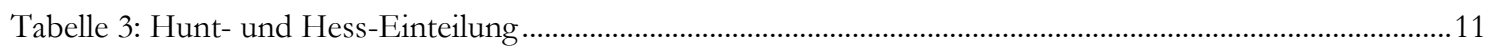

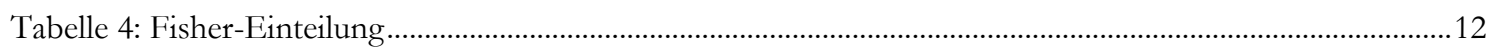

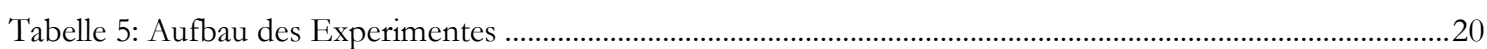




\begin{tabular}{ll}
\hline Abkürzungsverzeichnis \\
ACA & Arteria cerebri anterior \\
aSAB & Aneurysmatische Subarachnoidalblutung \\
CCT & Zerebrale Computertomographie \\
CTA & Computertomographische Angiographie \\
CTP & Computertomographische Perfusion \\
DINDs & Delayed ischemic neurological deficits \\
DSA & Digitale Subtraktionsangiographie \\
GCS & Glasgow Coma Scale \\
MCA & Arteria cerebri media \\
PCA & Arteria cerebri posterior \\
PET & Positronen-Emissions-Tomographie \\
SPECT & Single-Photon-Emissions-Computertomographie \\
TCD & Transkranielle Doppler-Sonographie \\
WFNS & World Federation of Neurosurgical Societies
\end{tabular}




\section{$1 \quad$ Einleitung}

Die Subarachnoidalblutung (SAB) ist eine schwerwiegende Form des hämorrhagischen Schlaganfalls, die im Vergleich zu den anderen Schlaganfallformen ein jüngeres Patientenkollektiv betrifft und oft mit einer schlechten Prognose einhergeht. Seit vielen Jahren ist die SAB Gegenstand der Forschung, wobei ein besonderer Schwerpunkt immer noch im Bereich der sogenannten verzögerten zerebralen Ischämie liegt. Die Etablierung verschiedener tierexperimenteller SAB-Modelle hat in den letzten Jahren zu einem erheblichen Zugewinn an Erkenntnissen im Bereich der Pathophysiologie der verzögerten zerebralen Ischämie beigetragen.

Mehrere klinische Klassifikationen/Graduierungen/Einteilungen der SAB wurden bisher definiert. Die Klassifikationen hängen mit prognostischen Faktoren zusammen und erlauben dadurch eine Abschätzung des Risikos für die Entwicklung von Komplikationen im Verlauf nach der Blutung und erleichtern die Therapieplanung. Die Etablierung solcher GradingSysteme für den Schweregrad der SAB ist ebenfalls im tierexperimentellen Bereich von großer Bedeutung, damit experimentelle Ergebnisse besser in den klinischen Bereich transferiert werden können.

\subsection{Fragestellung der Arbeit}

Während es verschiedene klinische Klassifikationen und Graduierungen für den Schweregrad der SAB gibt, wurden bisher keine Grading-Systeme zur Einteilung des Schweregrades der experimentellen SAB entwickelt. In der klinischen Praxis wird der FisherScore am häufigsten eingesetzt, um eine Einteilung der Schweregrade einer SAB vornehmen zu können.

Ziel dieser Arbeit ist die Etablierung eines Grading-Systems, welches analog zum FisherScore in der Humanmedizin die Einteilung der experimentellen SAB nach dem Schweregrad der Blutung im Rattenmodell ermöglicht.

Aus diesem Grund haben wir sowohl histologisch als auch bildgebend die Blutverteilung und die Blutmenge im SAB-Rattenmodell analysiert und anhand dessen ein Grading-System entwickelt. 


\subsection{Wissenschaftlicher Hintergrund}

\subsubsection{Subarachnoidalblutung (Definition, Epidemiologie und Ätiologie)}

Die SAB ist eine akute intrakranielle Blutung in den sogenannten Subarachnoidalraum (Abbildung 1 und 2), zwischen Arachnoidea mater und Pia mater, der mit Liquor cerebrospinalis (Hirnwasser) gefüllt ist. Die Blutung kann sich ebenfalls in die Hirnventrikel (Hirnwasserkammer), in den Subduralraum (Raum zwischen der Dura und der Arachnoidea mater) und in das Hirnparenchym (Hirngewebe) ausdehnen. Der Subarachnoidalraum beinhaltet sowohl die basalen Zisternen als auch die Sulci cerebri (Hirnfurchen) zwischen den Gyri cerebri (Hirnwindungen).

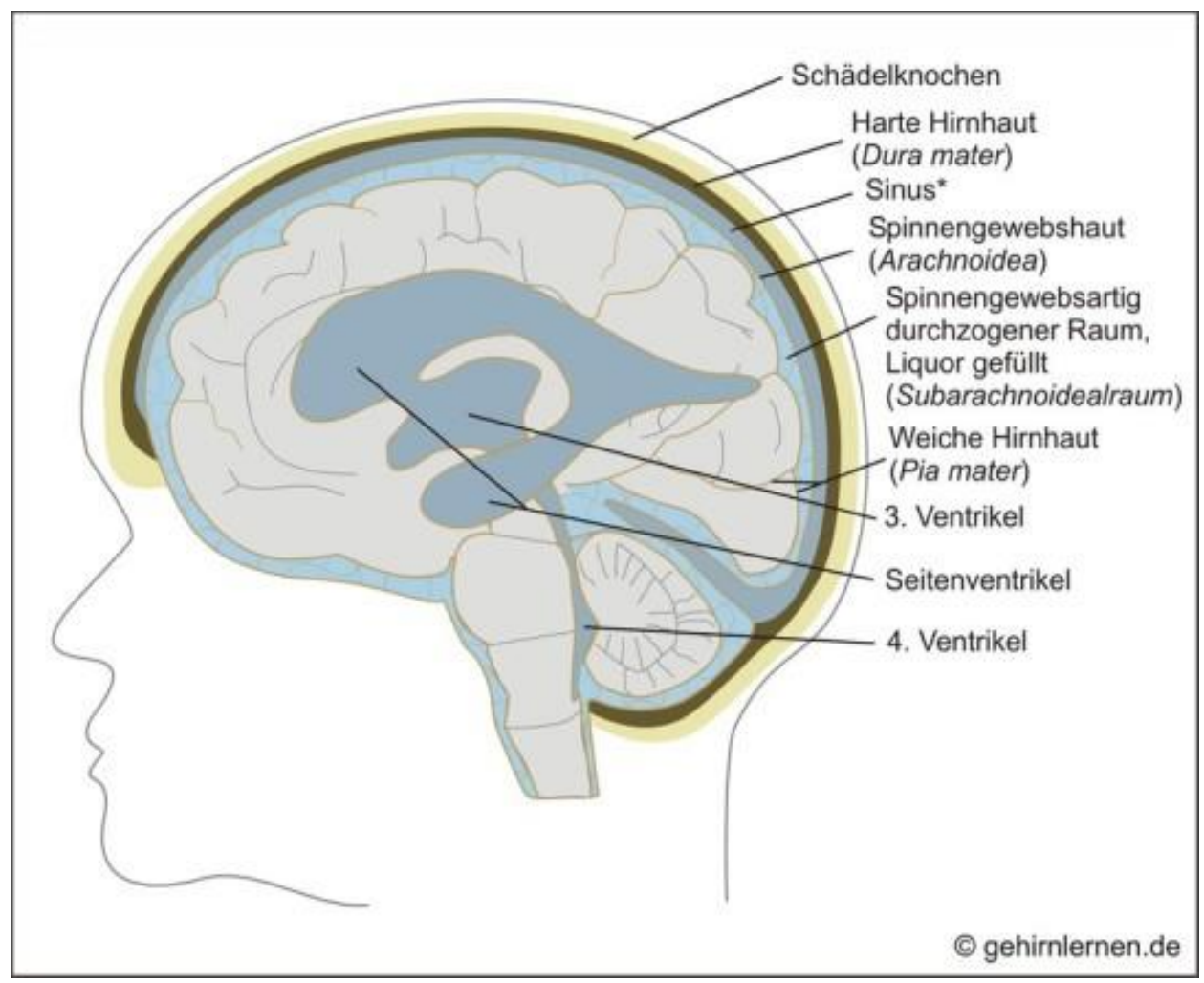

Abbildung 1. Basale Cisternen und Hirnventrikel (mit freundlicher Genehmigung von Dr. rer. nat. Andrea T. U. Schäfers, www.gehirnlernen.de) 
Die SAB macht ca. $5 \%$ aller Schlaganfälle aus. In Europa beträgt die Inzidenz der SAB 69/100000 Einwohner pro Jahr (DGN-Leitlinie SAB 2012). Frauen sind 1,6-mal häufiger betroffen als Männer. Das mittlere Lebensalter der Patienten zum Zeitpunkt der Erstmanifestation einer SAB liegt bei ca. 50 Jahren. Die SAB kann spontan auftreten oder traumatisch bedingt sein. In 85 \% der Fälle wird die spontane SAB durch die Ruptur eines Aneurysmas der zerebralen Arterien verursacht (aneurysmatische Subarachnoidalblutung = aSAB) (Van Gijn und Rinkel 2001, Leber at al. 2008, Schmieder et al. 2007, DGN-Leitlinie $\underline{\text { SAB 2012, D'Souza 2015). }}$.

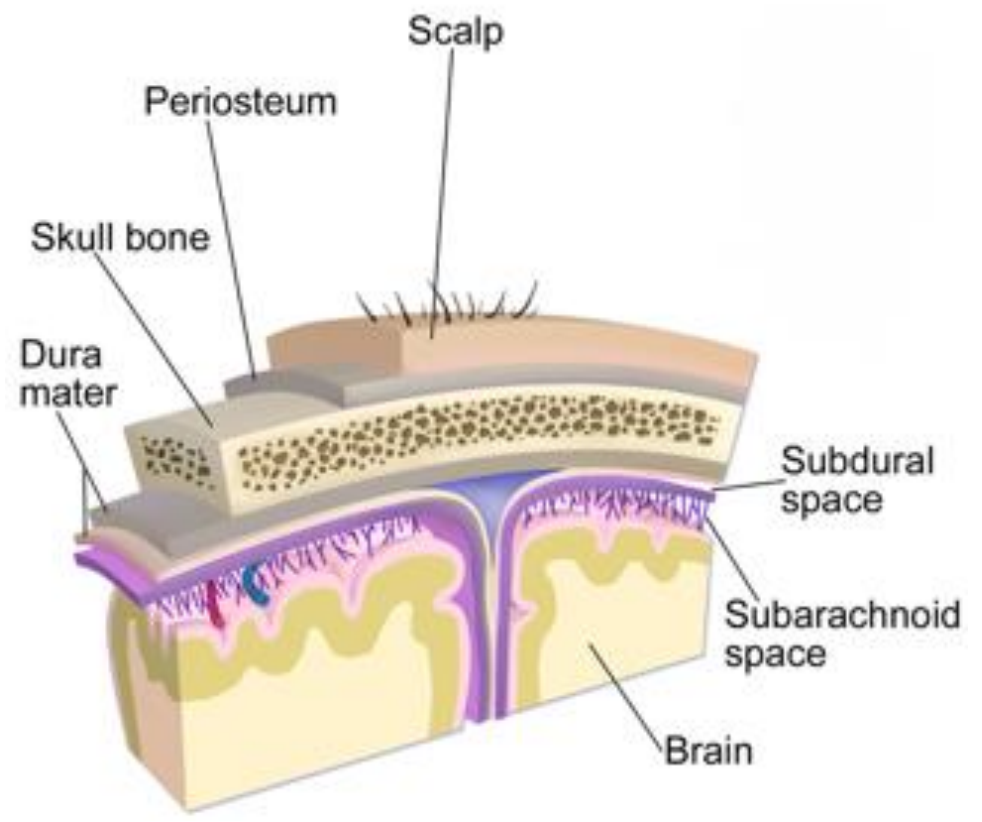

\section{Abbildung 2. Hirnhäute und Räume dazwischen (mit freundlicher Genehmigung des Redaktionsteams von www.lecturio.de)}

Aneurysmen sind Gefäßaussackungen an den Gefäßwänden, meistens an den Teilungsstellen der Arterien des Circulus arteriosus Willisi (Abb. 3). Der Circulus arteriosus Willisi liegt im Subarachnoidalraum innerhalb der basalen Zisternen. Wenn es zu einer Ruptur eines Aneurysmas kommt, verteilt sich das Blut um die basalen Arterien des Circulus arteriosus Willisi. Die Prävalenz von nicht-rupturierten (inzidentellen) Aneurysmen wird mit 1-9 \% angegeben (Etminan et al. 2015). In 15-20\% aller Patienten mit einer SAB sind mehrere Aneurysmen vorhanden. 


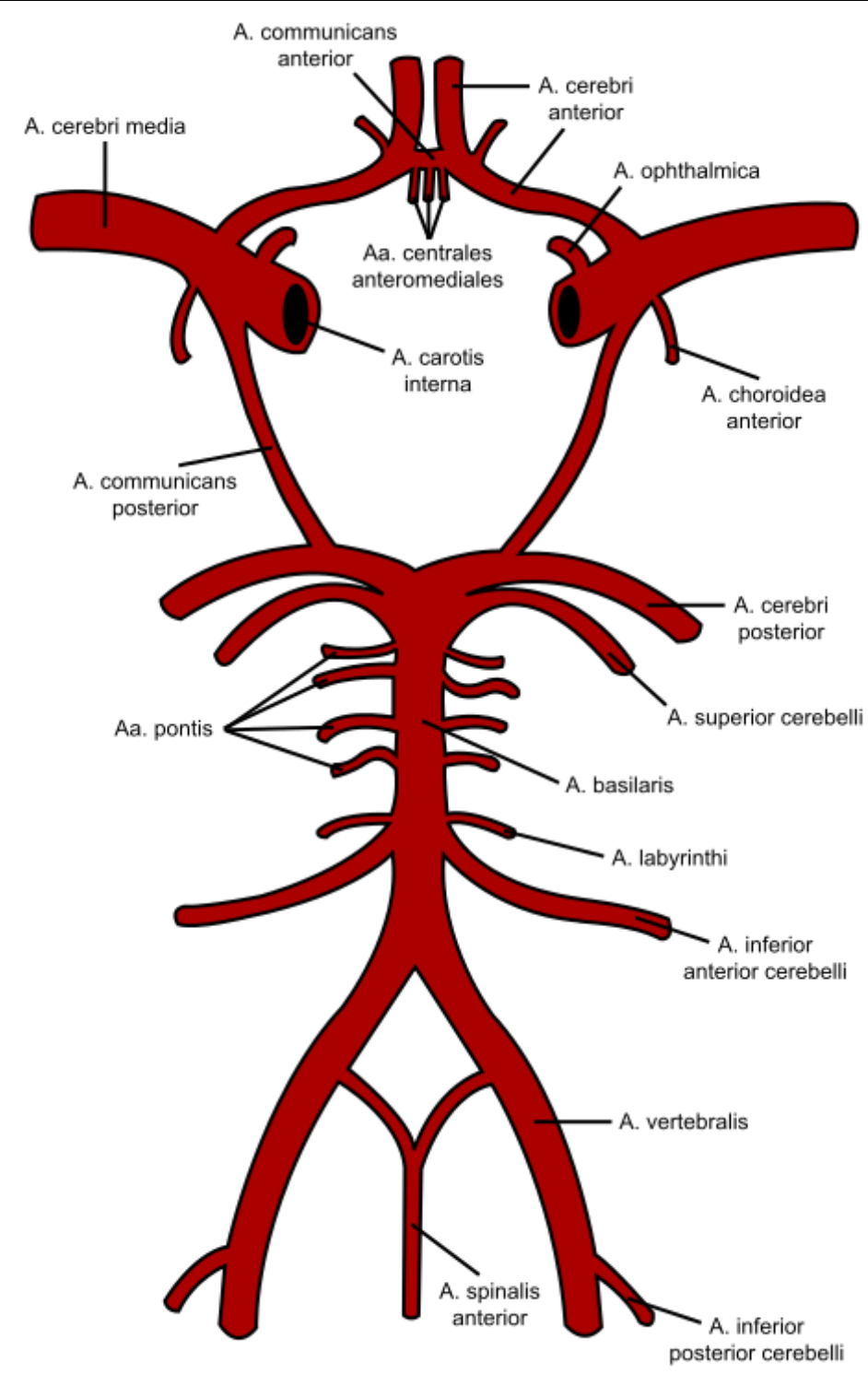

Abbildung 3. Circulus arteriosus Willisi (mit freundlicher Genehmigung von www.wikimedia.org)

Die Ruptur von Aneurysmen wird durch verschiedene Risikofaktoren begünstigt: Aneurysmagröße, Aneurysmalokalisation, Aneurysmawachstum, Anzahl der Aneurysmen, Patientenalter, Geschlecht der Patienten, arterielle Hypertonie (2,5-fach erhöhtes Risiko), Rauchen (2,3-fach erhöhtes Risiko), Alkoholkonsum (2-fach erhöhtes Risiko) sowie genetische Faktoren (Lindbohm et al. 2016, Feigin et al. 2005). Das Rupturrisiko inzidenteller Aneurysmen mit einer Größe von $>10 \mathrm{~mm}$ wird mit 1 \% pro Jahr und bei einer Größe von

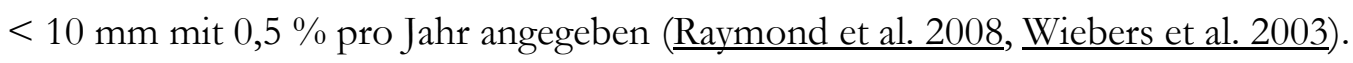


Zu den genetischen Syndromen mit erhöhtem Risiko für zerebrale Aneurysmen gehören: polyzystische Nieren, Ehlers-Danlos-Syndrom Typ 4, Marfan-Syndrom. Das Risiko für eine de-novo-Aneurysma-Entstehung liegt bei 1-2 \% pro Jahr. Nicht-rupturierte Aneurysmen, insbesondere wenn diese symptomatisch oder größenprogredient sind und im Bereich der A. communicans posterior oder im hinteren Stromkreislauf lokalisiert sind, bergen ein erhöhtes Risiko für eine $\mathrm{SAB}$, vor allem wenn eine $\mathrm{SAB}$ in der Vorgeschichte bekannt ist oder wenn es sich um Aneurysmen von Patienten mit familiärem Risiko handelt (mindestens 2 Familienangehörige ersten Grades, 20-fach erhöhtes Risiko) (Teasdale et al. 2005). Angehörige ersten Grades von SAB-Patienten haben ein geschätztes 10-Jahres-Risiko für das Auftreten einer SAB von 1,2 \% und Angehörige zweiten Grades von 0,5\%. Das lebenslange (bis zum 70ten Lebensjahr) Risiko von Angehörigen ersten Grades eine SAB zu bekommen wird auf 4,2 \% und von Angehörigen zweiten Grades auf 2,3\% geschätzt (Teasdale et al. 2005). Basierend auf diesen Daten wird ein Familienscreening nur empfohlen, wenn mindestens zwei Angehörige ersten Grades betroffen sind.

Zur Einschätzung des kumulativen 5-Jahres-Rupturrisikos eines inzidentellen Aneurysmas wurde der sogenannte PHASES Score entwickelt. Dieser Score berücksichtigt folgende Risikofaktoren: die ethnische Zugehörigkeit, das Vorhandensein eines arteriellen Hypertonus, das Alter des/der Patienten/in, die Größe und Lokalisation des Aneurysmas sowie eine aSAB in der Vorgeschichte (Greving et al. 2014). Die Berechnung des PHASES Score ist in Abbildung 4 dargestellt. Bei der Beratung von Patienten mit einem inzidentellen intrakraniellen Aneurysma müsste allerdings das Rupturrisiko gegen das Behandlungsrisiko des Aneurysmas abgewogen werden. Das Behandlungsrisiko wurde beim PHASES Score nicht berücksichtigt. Zu diesem Zweck wurde ein weiterer Score (UIATS = Unruptured intracranial aneurysm treatment score) erarbeitet, der patientenspezifische, aneurysmaspezifische sowie behandlungsspezifische Risikofaktoren vereint und einen multidisziplinären Konsens zur Behandlung von nicht-rupturierten Aneurysmen darstellt (Etminan et al. 2015). Der Erhebungsbogen des UIATS ist in Abbildung 5 dargestellt.

Zerebrale Aneurysmen entstehen typischerweise an den Aufzweigungsstellen (Bifurkationen) der zerebralen Arterien. An Gefäßbifurkationen kommt es häufig zu hämodynamischen Schwankungen. Es wird vermutet, dass dies bei Vorhandensein eines arteriellen Hypertonus zu einer Größenzunahme von Aneurysmen führt. Des Weiteren wird angenommen, dass Aneurysmen häufiger in der Phase der Größenzunahme aufgrund der dünner werdenden Aneurysmawand rupturieren (Juvela et al. 2001). 


\begin{tabular}{|c|c|}
\hline PHASES aneurysm risk score & Points \\
\hline \multicolumn{2}{|l|}{ (P) Population } \\
\hline North American, European (other than Finnish) & 0 \\
\hline Japanese & 3 \\
\hline Finnish & 5 \\
\hline \multicolumn{2}{|l|}{ (H) Hypertension } \\
\hline No & 0 \\
\hline Yes & 1 \\
\hline \multicolumn{2}{|l|}{ (A) Age } \\
\hline$<70$ years & 0 \\
\hline$\geq 70$ years & 1 \\
\hline \multicolumn{2}{|l|}{ (S) Size of aneurysm } \\
\hline$<7.0 \mathrm{~mm}$ & 0 \\
\hline $7 \cdot 0-9.9 \mathrm{~mm}$ & 3 \\
\hline $10 \cdot 0-19.9 \mathrm{~mm}$ & 6 \\
\hline$\geq 20 \mathrm{~mm}$ & 10 \\
\hline \multicolumn{2}{|l|}{ (E) Earlier SAH from another aneurysm } \\
\hline No & 0 \\
\hline Yes & 1 \\
\hline \multicolumn{2}{|l|}{ (S) Site of aneurysm } \\
\hline ICA & 0 \\
\hline MCA & 2 \\
\hline ACA/Pcom/posterior & 4 \\
\hline \multicolumn{2}{|c|}{$\begin{array}{l}\text { To calculate the PHASES risk score for an individual, the number of points } \\
\text { associated with each indicator can be added up to obtain the total risk score. For } \\
\text { example, a } 55 \text {-year-old North American man with no hypertension, no previous } \\
\text { SAH, and a medium-sized ( } 8 \mathrm{~mm} \text { ) posterior circulation aneurysm will have a risk } \\
\text { score of } 0+0+0+3+0+4=7 \text { points. According to figure } 3 \text {, this score corresponds to } \\
\text { a 5-year risk of rupture of } 2 \cdot 4 \% \text {. SAH=subarachnoid haemorrhage. ICA=internal } \\
\text { carotid artery. MCA=middle cerebral artery. ACA=anterior cerebral arteries } \\
\text { (including the anterior cerebral artery, anterior communicating artery, and } \\
\text { pericallosal artery). Pcom=posterior communicating artery. posterior=posterior } \\
\text { circulation (including the vertebral artery, basilar artery, cerebellar arteries, and } \\
\text { posterior cerebral artery). }\end{array}$} \\
\hline
\end{tabular}

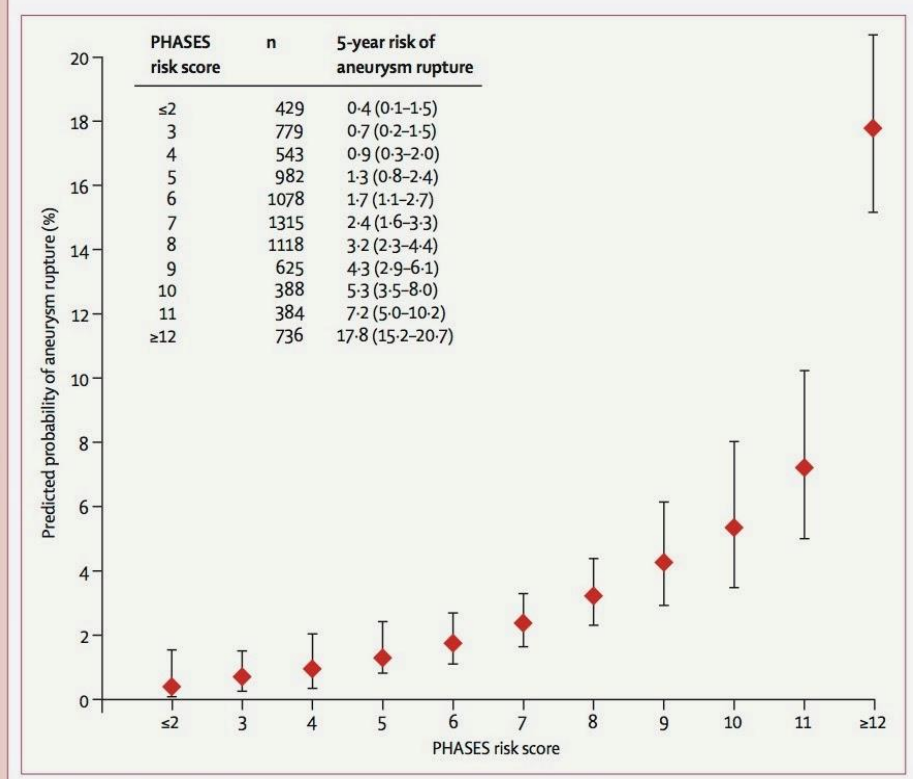

Figure 3: Predicted 5-year risk of aneurysm rupture according to PHASES score

Abbildung 4. PHASES Score (Greving et al. 2014) 


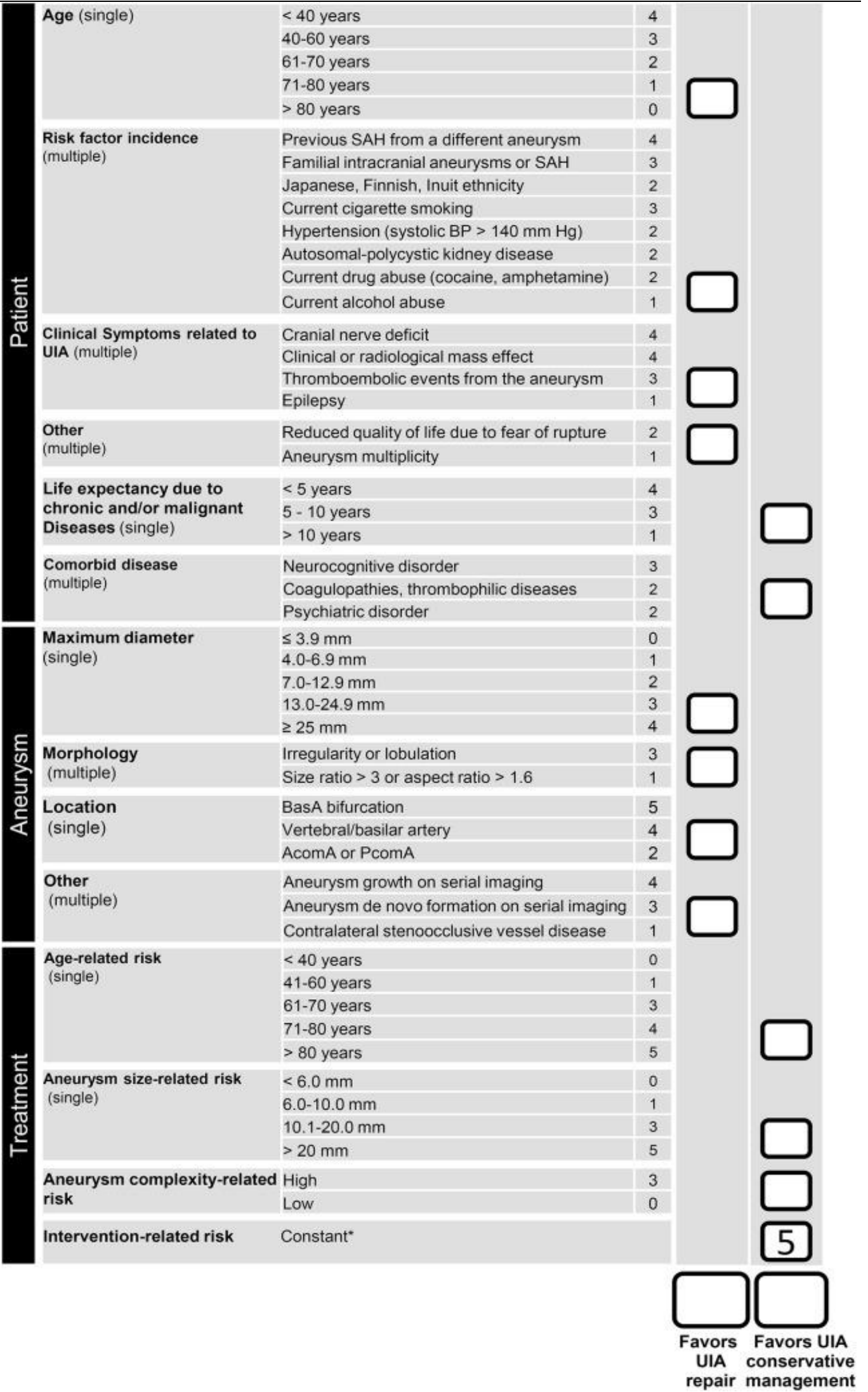

Abbildung 5. UIATS (Etminan et al. 2015) 
Die Lokalisation zerebraler Aneurysmen ist in $90 \%$ der Fälle im Bereich des vorderen Stromkreislaufes (A. cerebri anterior, A. communicans anterior, A. carotis interna, A. cerebri media und A. communicans posterior) und in $10 \%$ der Fälle im Bereich des hinteren Stromkreislaufes (A. vertebralis, A. basilaris, A. cerebelli superior, A. cerebelli inferior anterior, A. cerebelli inferior posterior).

\subsubsection{Klinik, Diagnostik und Klassifikation der SAB}

Das Hauptsymptom, mit dem sich eine $\mathrm{SAB}$ manifestiert, ist der sogenannte Vernichtungskopfschmerz (in $95 \%$ der Fälle). In $25 \%$ der Fälle kommt es vor dem Auftreten einer SAB zu einer sogenannten „Warnblutung = minor leak“, die sich mit heftigen Kopf- und Nackenschmerzen äußert. Diese bessern sich in den darauffolgenden Tagen, bevor es zu einer schwerwiegenden SAB kommt (Jakobsson et al. 1996). Zusätzliche Symptome können Meningismus (Nackensteifigkeit), Paresen (Lähmungen), eine Sprachstörung, Bewusstseinsstörung sowie vegetative Symptome wie Übelkeit und Erbrechen sein. In $25 \%$ der Fälle manifestiert sich die SAB mit einem Krampfanfall (Schmieder et al. 2007, Edlow und Caplan 2000).

Die Durchführung einer zerebralen Computertomographie (CCT) ohne Kontrastmittel ist die Methode der Wahl zur Diagnostik einer SAB. Ob die SAB in der CCT nachgewiesen werden kann, hängt von der Ausprägung der Blutung und von dem Zeitpunkt der Untersuchung ab. Am Tag des Symptombeginns kann eine SAB in $92 \%$ in der CCT nachgewiesen werden (Leber et al. 2008, Carpenter et al. 2016).

Bei SAB-typischen klinischen Symptomen und bei gleichzeitig fehlendem Blutungsnachweis in der CCT muss eine Lumbalpunktion zum Ausschluss einer SAB durchgeführt werden. Die Lumbalpunktion sollte so früh wie möglich durchgeführt werden, bestenfalls innerhalb von 6-12 Stunden nach Symptombeginn. Nach 12 Stunden beginnt der Erythrozytenzerfall, der zu einer Xanthochromie des Liquors führt. Erythrozyten und Siderophagen können noch mehrere Tage nach dem Blutungsereignis im Liquor nachgewiesen werden. Nach ca. einer Woche sind bereits $90 \%$ des Blutes resorbiert (Carpenter et al. 2016).

Beim Nachweis einer SAB mittels CCT sollte zur weiteren diagnostischen Abklärung zusätzlich die Darstellung der zerebralen Gefäße erfolgen. Zu diesem Zweck kann eine CTAngiographie durchgeführt werden, in der Aneurysmen ab einer Größe von $3 \mathrm{~mm}$ nachgewiesen werden können. Die konventionelle Angiographie bzw. digitale Subtraktionsangiographie (DSA) stellt weiterhin die Diagnostik der Wahl dar. Falls die erstmalige DSA zum Zeitpunkt der Manifestation der SAB keinen Aneurysmanachweis 


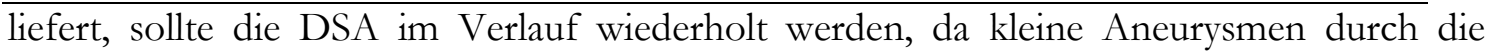
Blutung in der initialen Phase unentdeckt bleiben können.

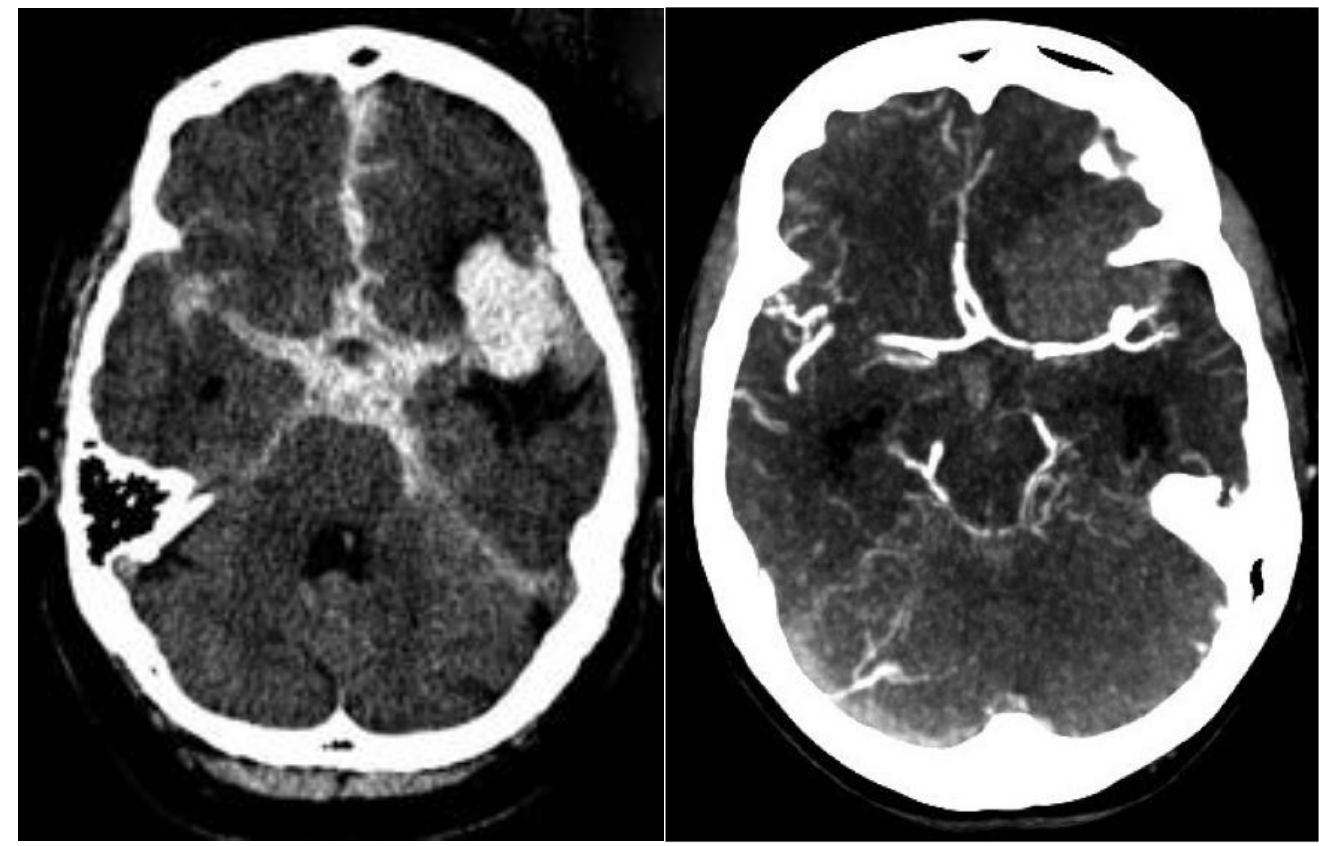

Abbildung 6. Aneurysmatische Subarachnoidalblutung (native

Computertomographie und computertomographische Angiographie) - mit freundlicher Genehmigung der Abteilung für Neuroradiologie der UMG (Klinikdirektor Prof. Dr. M. Knauth)

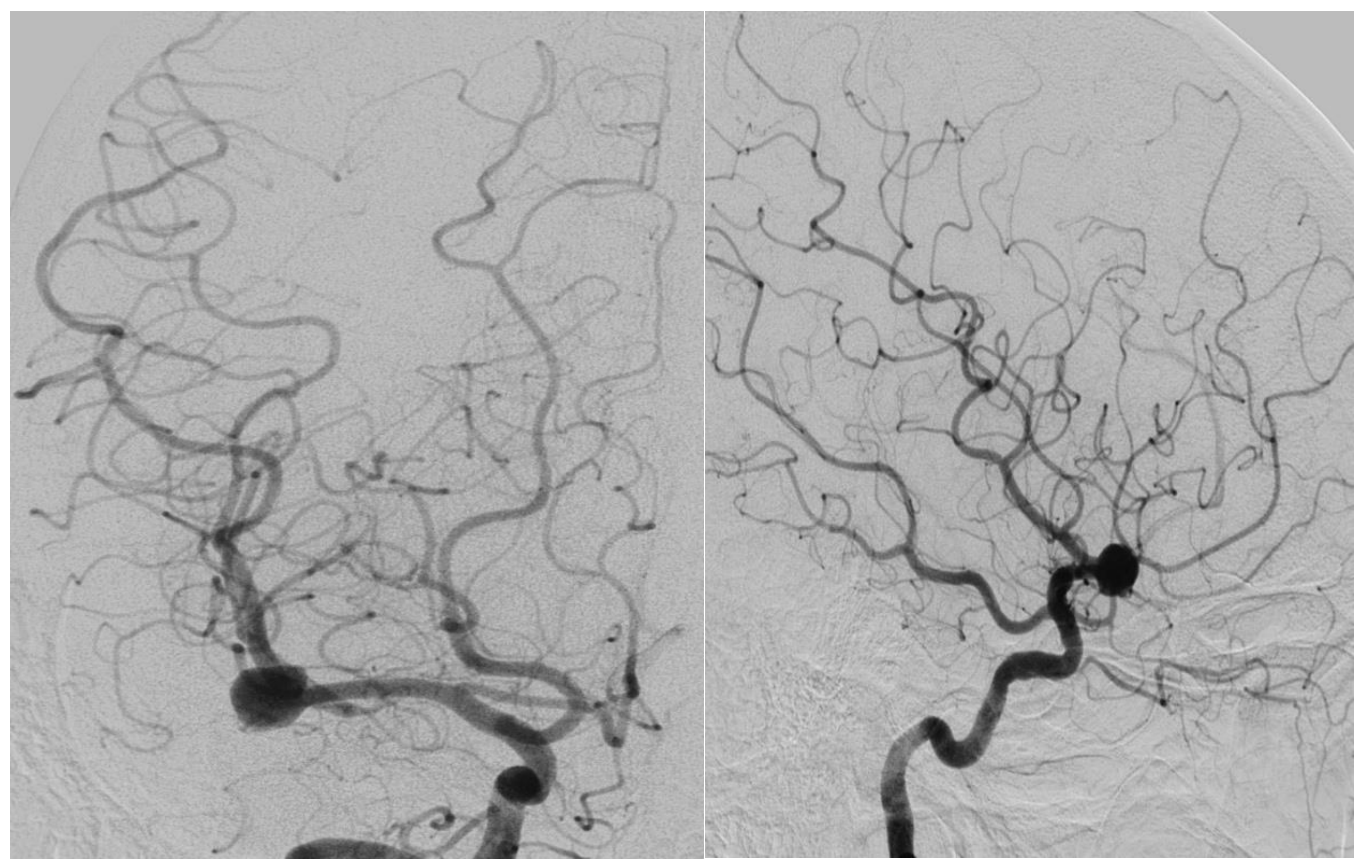

Abbildung 7. DSA mit einem Aneurysma an der A. cerebri media rechts - mit freundlicher Genehmigung der Abteilung für Neuroradiologie der UMG (Klinikdirektor Prof. Dr. M. Knauth) 
Der klinische Status der Patienten bei der Manifestation einer SAB wird anhand der Huntund Hess-Skala ( Neurological Societies) - Skala erfasst. Die WFNS-Skala basiert auf der Glasgow Coma Skala (GCS).

Die GCS wurde im Jahr 1974 zur Beurteilung des Bewusstseins der Patienten mit SchädelHirn-Trauma vorgestellt (Teasdale 1972). Diese Skala beinhaltet die Beurteilung von drei klinischen Reaktionen des Patienten: 1) das Öffnen der Augen, 2) die verbale und die 3) motorische Reaktion. Die Tabelle 1 zeigt die Auflistung der Antworten nach denen die GCS berechnet wird. In der Tabelle 2 ist die Einteilung nach der WFNS-Skala dargestellt (Teasdale et al. 1988).

Tabelle 1. GCS-Skala (Teasdale 1972)

\begin{tabular}{|c|c|c|c|c|c|}
\hline \multicolumn{2}{|c|}{ Augen Öffnen } & \multicolumn{2}{|c|}{ Verbale Reaktion } & \multicolumn{2}{|c|}{ Motorische Reaktion } \\
\hline Keine Antwort & 1 Punkt & Keine Antwort & 1 Punkt & Keine Antwort & 1 Punkt \\
\hline Auf Schmerzreiz & 2 Punkte & Unverständliche Laute & 2 Punkte & Strecksynergismen & 2 Punkte \\
\hline Auf Ansprache & 3 Punkte & Einzelne Worte & 3 Punkte & Beugesynergismen & 3 Punkte \\
\hline \multirow[t]{3}{*}{ Spontan } & 4 Punkte & Desorientiert & 4 Punkte & Ungezielte Abwehr & 4 Punkte \\
\hline & & Orientiert & 5 Punkte & Gezielte Abwehr & 5 Punkte \\
\hline & & & & Befolgt Aufforderung & 6 Punkte \\
\hline
\end{tabular}

Tabelle 2. WFNS-Skala (Teasdale et al. 1988)

\begin{tabular}{|l|l|l|}
\hline WFNS Grad & GCS & Klinik \\
\hline 1 & 15 & Ohne motorisches Defizit \\
\hline 2 & $13-14$ & Ohne motorisches Defizit \\
\hline 3 & $13-14$ & Mit motorischem Defizit \\
\hline 4 & $7-12$ & Mit oder ohne motorisches Defizit \\
\hline 5 & $3-6$ & Mit oder ohne motorisches Defizit \\
\hline
\end{tabular}


Die Hunt- und Hess-Skala richtet sich nach dem Vorhandensein von Kopfschmerzen und Nackensteifigkeit auf der einen Seite sowie nach dem Vorhandensein neurologischer Defizite und nach der Bewusstseinslage des Patienten auf der anderen Seite. Die klinische Einteilung der SAB nach der Hunt- und Hess-Skala erlaubt die Vorhersage der Mortalität (Grad-I 0-15 \%, Grad-II 5-10 \%, Grad-III 15-35 \%, Grad-IV 30-40 \% und Grad-V 50-90 \%) (Connolly et al. 2012, De Oliveira Manoel et al. 2015, Rosen und Macdonald 2005, Cavanagh und Gordon 2002, Van Norden et al. 2006).

Tabelle 3. Einteilung nach Hunt und Hess 1968

\begin{tabular}{|l|l|}
\hline Grad & Klinik \\
\hline 1 & $\begin{array}{l}\text { Asymptomatisch oder leichter Kopfschmerz, } \\
\text { leichter Meningismus }\end{array}$ \\
\hline $1 \mathrm{a}$ & $\begin{array}{l}\text { Asymptomatisch oder nur geringe Kopfschmerzen } \\
\text { und/oder Meningismus, jedoch mit festen } \\
\text { neurologischen Defiziten }\end{array}$ \\
\hline 2 & $\begin{array}{l}\text { Hirnnervenausfälle, mäßige bis schwere } \\
\text { Kopfschmerzen, Meningismus }\end{array}$ \\
\hline 3 & $\begin{array}{l}\text { Leichte fokal-neurologische Defizite, } \\
\text { Desorientierung, Vigilanzminderung (Somnolenz) }\end{array}$ \\
\hline 4 & Stupor, mäßige bis schwere Hemiparese \\
\hline 5 & Koma, Dezerebration \\
\hline
\end{tabular}

Die Schwere der SAB wird anhand der Blutmenge und der Blutverteilung in der initialen CCT mit Hilfe der Fisher-Skala klassifiziert (Fisher et al. 1980). Diese Skala, welche ursprünglich im Jahr 1980 vorgestellt wurde, ist im Verlauf mehrfach revidiert und modifiziert worden (Frontera et al. 2006, Claassen et al. 2001, Hijdra et al. 1990). Bis heute ist die Fisher-Skala die am häufigsten im klinischen Alltag sowie in klinischen Studien eingesetzte Klassifikation zur Beurteilung der Schwere einer SAB. Frühere Studien konnten zeigten, dass diese Einteilung mit der Prognose sowie mit dem Auftreten von 
Komplikationen im Verlauf nach einer SAB korreliert (Frontera et al. 2006, Claassen et al. 2001).

Tabelle 4. Fisher-Einteilung (Fisher et al. 1980)

\begin{tabular}{|l|l|}
\hline Fisher-Grad & Blutmenge und Verteilung \\
\hline 1 & Kein subarachnoidales Blut \\
\hline 2 & Diffuse $<1 \mathrm{~mm}$ dicke SAB \\
\hline 3 & Lokalisierter Blutclot und/oder $>1 \mathrm{~mm}$ dicke SAB \\
\hline 4 & Intrazerebrale oder intraventrikuläre Blutung mit diffuser SAB \\
\hline
\end{tabular}

\subsubsection{Frühe Hirnschädigung (early brain injury) nach einer SAB}

Im Verlauf nach einer SAB können multiple Komplikationen sowohl in der frühen Phase als auch im späteren Verlauf auftreten. Die SAB führt zu einer unmittelbaren Hirnschädigung, die in der initialen Phase nach der Manifestation der Blutung entsteht und als early brain injury bezeichnet wird. Nach der Aneurysmaruptur kommt es zu einem plötzlichen Anstieg des intrakraniellen Druckes, was wiederum zu einer Störung der Autoregulation des Gehirns mit einem Abfall des zerebralen Perfusionsdruckes und des zerebralen Blutflusses führt und in einer Minderdurchblutung des Gehirns (globale zerebrale Ischämie) mündet. Durch die Ischämie kommt es zu einem Neuronenverlust, was die Entstehung eines zytotoxischen Ödems (Hirnschwellung) begünstigt. Die Schädigung der Endothelzellen wiederum ist verantwortlich für die Induktion eines vasogenen Ödems. Diese Prozesse zum Zeitpunkt des Blutungsereignisses aktivieren weitere Vorgänge wie z. B. die Gerinnungskaskade und die Neuroinflammation und können der Initiator von einer sekundären Hirnschädigung im Verlauf nach einer SAB sein (Fujii et al. 2013). Im Rahmen dieser frühen Hirnschädigung werden sowohl die Hirngefäße als auch das Hirnparenchym geschädigt.

\subsubsection{Verzögerte zerebrale Ischämie (delayed cerebral ischemia) nach einer SAB}

Früher war das Auftreten eines zerebralen Vasospasmus (Gefäßeinengung der zerebralen Arterien) die meist gefürchtete Komplikation nach einer SAB (Ciurea et al. 2013). Einen besonderen Stellenwert unter den möglichen Komplikationen nach einer SAB, auch als Folge 
zerebraler Vasospasmen, hat die verzögerte zerebrale Ischämie. Diese kann zeitversetzt Infarkte und damit einhergehende permanente neurologische Defizite verursachen und folglich die Prognose einer SAB negativ beeinflussen.

Nach neuesten Erkenntnissen der Forschung aus den letzten Jahren wissen wir, dass der Vasospasmus nur einen von multiplen Faktoren, welche zu einer verzögerten zerebralen Ischämie (delayed cerebral ischemia) führen können, darstellt (Inagawa et al. 2014, Foreman 2016, Nakaoka et al. 2014, Vergouwen et al. 2008, Zheng et al. 2012).

Zerebrale Vasospasmen, die in der konventionellen Angiographie nachgewiesen werden können, treten in bis zu $70 \%$ der Patienten mit einer SAB auf. Zusätzlich zu den proximalen Vasospasmen, welche größere (proximale) Abschnitte der Gefäße betreffen, existieren ebenfalls distale Vasospasmen, die bis zu den Arteriolen des Gehirns reichen und sich mit der Angiographie in der Regel nicht suffizient darstellen lassen. Abhängig von dem Schweregrad der Vasospasmen (niedriggradig, mittelgradig, schwerwiegend) kommt es lokal zu einer Verminderung der Hirndurchblutung. In $30 \%$ dieser Fälle treten im Verlauf verzögerte neurologische Defizite (delayed ischemic neurological deficits $=$ DINDs), als symptomatische Vaospasmen bezeichnet, auf. Der symptomatische Vasospasmus wird definiert als neu aufgetretenes fokal-neurologisches Defizit, Vigilanzminderung oder beides, nachdem andere mögliche Ursachen, wie ein Hydrozephalus, epileptische Anfälle, metabolische Störungen, Infektionen oder eine persistierende Sedierung ausgeschlossen werden konnten (Frontera et al. 2009). Am Ende kommt es in 3-7 \% der Fälle zu verzögerten Infarkten. Verzögerte zerebrale Ischämien treten in der Zeit zwischen Tag 4 und Tag 14 nach dem Blutungsereignis ein.

Die Abbauprodukte des Blutes im Subarachnoidalraum wurden als Ursache für eine Vasokonstriktion durch Endothelhypertrophie betrachtet. Die genaue Pathophysiologie der verzögerten zerebralen Ischämie ist trotz umfangreicher Forschung immer noch nicht endgültig geklärt (Foreman 2016). Es wurden erhöhte Konzentrationen von Endothelin-1 (starker Vasokonstriktor) und ein Mangel an eNOS (endotheliale Stickstoffmonoxid Synthase) bei Patienten, die Vasospasmen im Verlauf nach einer SAB entwickeln, festgestellt (Hendrix et al. 2017). Es wurden in den letzten Jahren mehrere Theorien zur Erklärung der Pathomechanismen, welche zu einer verzögerten zerebralen Ischämie führen, beschrieben (Nakaoka et al. 2014, Vergouwen et al. 2008, Zheng et al. 2012).

Der akuten Hirnschädigung (early brain injury), die innerhalb der ersten 72 Stunden nach Manifestation einer SAB eintritt, wird hierbei ebenfalls eine besondere Bedeutung beigemessen (Rowland et al. 2012). Es wird postuliert, dass pathophysiologische 
Veränderungen in dieser Phase zu späterem Auftreten ischämischer Komplikationen nach einer SAB beitragen. Während der Aneurysmaruptur tritt Blut mit hohem Druck in den Subarachnoidalraum über, was zu einem schnellen Anstieg des intrakraniellen Druckes führt. Infolge dessen kommt es zu einem Abfall des zerebralen Blutflusses und zum sukzessiven Abfall des zerebralen Perfusionsdruckes. Zusätzlich kommt es zu einem akuten Hydrozephalus (Wasserkopf) durch eine Abflussstörung des Liquors, bedingt durch das sich verteilende Blut in den basalen Zisternen, was ebenfalls zu einer Erhöhung des intrakraniellen Druckes führen kann (Rowland et al. 2012, Francoeur und Mayer 2016, Budohoski et al. 2014, De Rooij et al. 2013, Dupont et al. 2009). Die Blutabbauprodukte können ebenfalls eine Kaskade von Reaktionen in Gang setzen, wobei es zur Freisetzung von multiplen pro-inflammatorischen und vasoaktiven Faktoren kommt, die im Liquor nachgewiesen werden können. Des Weiteren kommt es als Reaktion auf die Blutung zu einer Aktivierung des Gerinnungssystems. In Post-mortem-Studien konnten vermehrt Mikrothrombosen bei Patienten mit SAB, assoziiert mit multiplen kortikalen ischämischen Läsionen, nachgewiesen werden. Folglich können Mikrothrombosen in der Pathogenese der verzögerten zerebralen Ischämie eine Rolle spielen (Vergouwen et al. 2008). Ein weiterer möglicher Faktor in der Pathogenese der verzögerten zerebralen Ischämie ist die kortikale Streudepolarisation (cortical spreading depression). Hierbei handelt es sich um ein Phänomen, bei dem es zu einer langsam ausbreitenden Depolarisation der Hirnrinde kommt mit daraus resultierenden Gefäßveränderungen. Es kommt zu einer Vasokonstriktion kurz vor Beginn der Welle sowie einer Vasodilatation während der Ausbreitung der Welle, gefolgt von einer verzögerten Minderdurchblutung des Hirngewebes (Rowland et al. 2012, Sehba et al. 2011, Dreier 2011, Dreier et al. 2017).

Die verzögerte zerebrale Ischämie ist eine potentiell vermeidbare Komplikation nach einer $\mathrm{SAB}$, vorausgesetzt, die Patienten, die ein erhöhtes Risiko dafür haben, wurden frühzeitig identifiziert sowie präventive und therapeutische Maßnahmen rechtzeitig eingeleitet. Es werden mehrere Methoden zur Diagnosesicherung der verzögerten zerebralen Ischämie angewendet.

Die klinisch-neurologische Untersuchung ist die verlässlichste Methode, um die Folgen einer verzögerten zerebralen Ischämie zu diagnostizieren. Diese kann allerdings nur bei wachen Patienten, welche Aufforderungen befolgen können, durchgeführt werden. Patienten mit einer $\mathrm{SAB}$ sind allerdings oft komatös oder müssen sediert werden, sodass eine klinischneurologische Untersuchung nicht immer möglich ist. Daher ist man bei diesen Patienten 
auf weitere technische Methoden in der Diagnostik angewiesen. Zudem sind bei ca. $20 \%$ der Patienten verzögerte zerebrale Ischämien asymptomatisch (Connolly et al. 2012).

Die transkranielle Doppler-Sonographie (TCD) ist eine nicht-invasive Methode, welche am Patientenbett durchgeführt werden kann und die Blutflussgeschwindigkeiten der großen basalen zerebralen Arterien misst. Eine Beschleunigung der Blutflussgeschwindigkeit über $120 \mathrm{~cm} / \mathrm{s}$ gemessen mittels TCD wird als Vasospasmus definiert. (Francoer et al. 2016). Der Lindegaard-Index (pathologisch wenn > 3), welcher das Verhältnis des zerebralen Blutflusses der Arteria cerebri media und der Arteria carotis communis beschreibt, soll weniger von systemischen hämodynamischen Variationen beeinflusst werden und die Möglichkeit der Verwechslung mit einer zerebralen Hyperperfusion reduzieren. Zudem ist die TCD untersucherabhängig und in ca. $10 \%$ der Fälle durch ein eingeschränktes Schallfenster limitiert. Die TCD hat insgesamt eine niedrige Sensitivität und Spezifität für die Diagnostik der verzögerten zerebralen Ischämie (Carrera et al. 2009). Weiterhin führen arteriosklerotische Veränderungen der Gefäße, somit insbesondere bei älteren Patienten, zu einer Einschränkung der Gefäßwandmotilität. Altersspezifische Grenzwerte der gemessenen Blutflussgeschwindigkeiten wurden bisher nicht definiert (Malinova et al. 2016a).

Die digitale Subtraktionsangiographie (DSA) ist der Goldstandard für die Diagnostik von zerebralen Vasospasmen. Die DSA birgt aber die Risiken einer invasiven Untersuchung mit einer Komplikationsrate für Dissektionen und Thromboembolien von 1-3\% ( 1999). Angiographisch wird eine Lumeneinengung von mehr als $50 \%$ als Vasospasmus bezeichnet. Eine nicht invasive Alternative zur DSA ist die CT-Angiographie (CTA). Diese ist allerdings auch wie die DSA mit einer Strahlenbelastung verbunden (De Oliveira Manoel et al. 2014).

Die verzögerte zerebrale Ischämie ist mit einer Hypoperfusion des Gehirns assoziiert. Verschiedene Methoden zur Untersuchung der zerebralen Perfusion wurden bisher zur Diagnostik der verzögerten zerebralen Ischämie eingesetzt. Hierzu gehören die Kernspintomographie, die Xenon-Computertomographie (Xenon-CT), PET (PositronenEmissions-Tomographie), SPECT (Single-Photon-Emissions-Computertomographie) und die CT-Perfusion (CTP) (De Oliveira Manoel et al. 2014, Malinova et al. 2016b). Des Weiteren ist ein multimodales invasives Monitoring sowohl des intrakraniellen Druckes (intracranial pressure $=$ ICP), der Oxygenierung des Hirngewebes, des zerebralen Blutflusses (cerebral blood flow $=\mathrm{CBF}$ ) sowie des zerebralen Metabolismus (Mikrodialyse) Teil der klinischen Routine (Helbok et al. 2015). 


\subsubsection{Therapie der SAB und ihrer Komplikationen}

Die Therapie der SAB besteht einerseits aus der Versorgung des rupturierten Aneurysmas und anderseits aus der Behandlung von frühen und späten Komplikationen, die im Verlauf der Erkrankung auftreten können. Die Ausschaltung des Aneurysmas erfolgt in der Regel entweder interventionell mittels endovaskulärem Coiling oder mikrochirurgisch durch Clipping (Abbildung 9). Beim endovaskulären Coiling (Abbildung 10) werden über einen Katheter Platinspiralen in das Aneurysma eingeführt. Ziel ist die vollständige Coiloklussion des Aneurysmas mit Induktion einer Thrombosierung des Aneurysmasacks. Durch die Thrombosierung wird das Aneurysma vom Blutkreislauf abgekoppelt und folglich die Blutungsquelle eliminiert. Im Gegensatz dazu wird beim mikrochirurgischen Clipping das Aneurysma mit einem Titan-Clip verschlossen.

In den letzten Jahren wurden mehrere Studien durchgeführt, welche die beiden Techniken miteinander verglichen haben. Die sogenannte ISAT-Studie (ISAT=International Subarachnoid Aneurysm Trial) ist zu diesem Thema die meist zitierte Studie. In diese Studie wurden 2143 Patienten eingeschlossen (Molyneux et al. 2002). Nach dieser Studie war das Coiling mit einer besseren Prognose nach einem Jahr assoziiert verglichen mit dem Clipping. Diese Studie weist allerdings einige Schwächen auf. Ein Kritikpunkt ist es, dass die endovaskuläre Behandlung der Aneurysmen in hochspezialisierten neuroradiologischen Zentren durchgeführt wurde. Im Gegensatz dazu wurden bei den chirurgisch behandelten Aneurysmen nicht gleich hohe Anforderungen an die behandelnden neurochirurgischen Zentren gestellt. Das kann einen Einfluss auf die Behandlungsergebnisse beider Gruppen haben. Ein weiterer Schwachpunkt dieser Studie ist, dass nur $22 \%$ der Patienten, welche randomisiert wurden, tatsächlich die zugewiesene Behandlung erhalten haben. Des Weiteren ist die Zeit bis zur Durchführung der zugewiesenen Behandlung zwischen den beiden Behandlungsgruppen unterschiedlich gewesen. Dabei war diese Zeit deutlich länger in der Clipping-Gruppe im Vergleich zur Coiling-Gruppe. Durch diese zeitliche Verzögerung kam es häufiger zu Nachblutungen in der Clipping-Gruppe, bevor die Aneurysma-Behandlung stattgefunden hat, was ebenfalls einen Einfluss auf die Prognose hat. Im Jahr 2015 wurden die Daten nach einem 10-Jahres-Follow-Up nach der ersten Veröffentlichung der Daten der ISAT-Studie veröffentlicht (Molyneux et al. 2015). Diese Studie zeigte, dass die Prognose der Patienten zwischen den beiden Behandlungsgruppen (Clipping und Coiling) vergleichbar ist. Die Re-Blutungsrate der behandelten Aneurysmen war deutlich größer in der CoilingGruppe verglichen zur Clipping-Gruppe, allerdings hatte dies keinen negativen Einfluss auf die Prognose der Patienten.

Ein kürzlich publiziertes systematisches Review inklusive einer Metaanalyse bescheinigte beiden Methoden eine vergleichbare Wertigkeit ohne statistisch signifikante Unterschiede

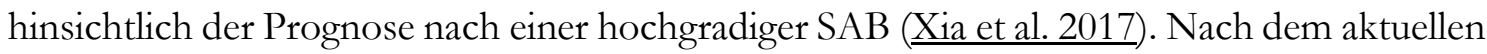
Wissensstand muss bei jedem Patienten individuell evaluiert werden, welche der beiden Methoden die geeignete Behandlung darstellt. Dabei werden verschiedene Faktoren wie die 
Aneurysmagröße, die Konfiguration des Aneurysmas, die Aneurysmalokalisation usw. berücksichtigt. Es wird empfohlen, bei allen Patienten mit einer aSAB eine interdisziplinäre Entscheidung zwischen den behandelnden Neurochirurgen und den behandelnden Neuroradiologen bezüglich der Aneurysmaversorgung unter Berücksichtigung der individuellen Konstellation zu treffen.

Während die Frage nach der besten Methode für die Aneurysmaversorgung noch kontrovers diskutiert wird, ist die Frage des Zeitpunktes der Aneurysmaversorgung nach einer aSAB klar. Das Aneurysma sollte nach einer aSAB innerhalb von 72 Stunden ausgeschaltet werden, um das Risiko einer Nachblutung zu reduzieren (Kassell et al. 1990a, Kassell et al. 1990b).
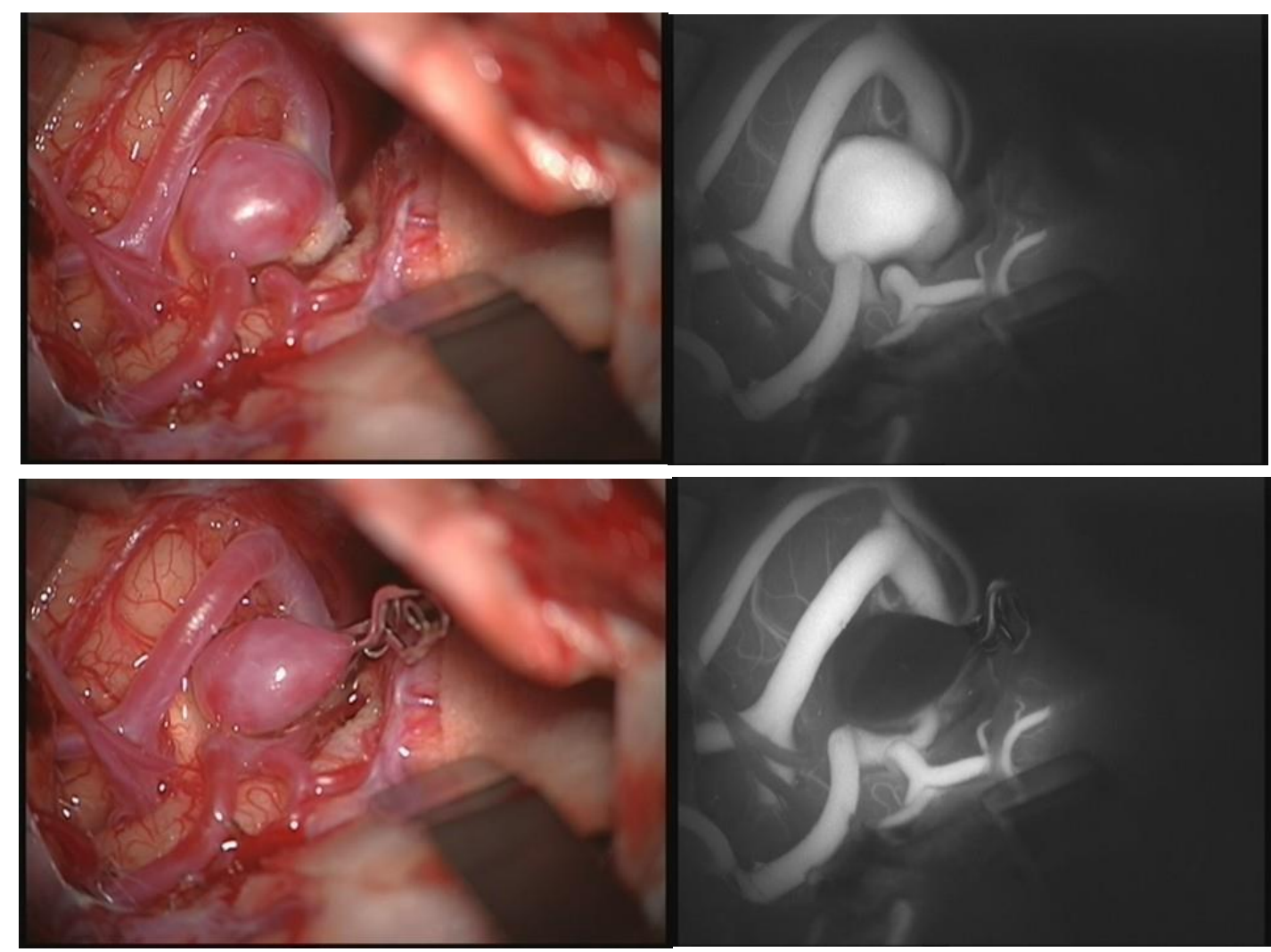

Abbildung 8. Operationssitus unter dem Mikroskop vor und nach Clipping eines Aneurysmas jeweils dargestellt unter Weißlicht und Infrarotlicht nach Injektion von Indocyanin-Grün (ICG) - (mit freundlicher Genehmigung von Dr. med. Kajetan von Eckardstein) 


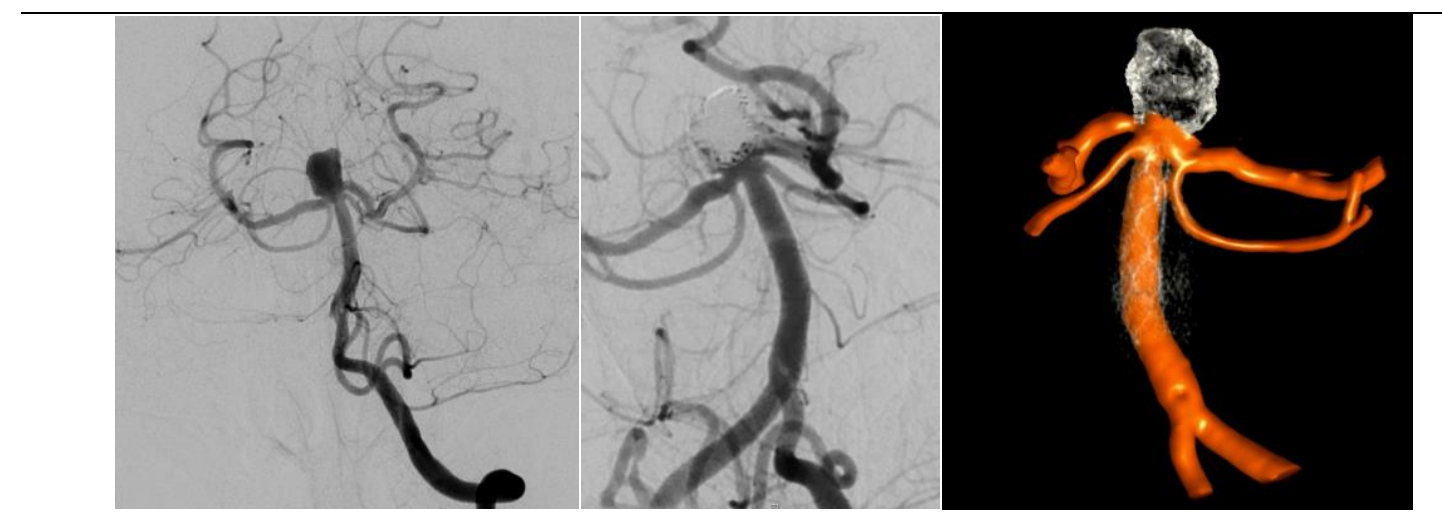

\section{Abbildung 9. Darstellung eines Basilariskopfaneurysmas vor und nach Coiling (mit freundlicher Genehmigung der Abteilung für Neuroradiologie der UMG} (Klinikdirektor Prof. Dr. M. Knauth)

\subsubsection{Prognose der SAB}

Trotz der vielen Fortschritte in der Medizin hat die SAB weiterhin eine schlechte Prognose mit einer hohen Morbidität und Mortalität (30-Tage-Letalität 35 \%) (Feigin et al. 2009). Etwa ein Drittel der Patienten mit einer SAB versterben, bevor sie das Krankenhaus erreichen. Ein Drittel der überlebenden Patienten, welche im Krankenhaus behandelt werden, sterben im Krankenhaus, ein Drittel behält nach der Entlassung eine dauerhafte Behinderung und nur ein Drittel der Patienten kann das Krankenhaus ohne bleibende Behinderung verlassen (Latigua et al. 2015, Szklener et al. 2015).

In einer Studie über die Lebensqualität nach einer SAB hatten vier Monate nach der Blutung $19 \%$ der Patienten eine gute Lebensqualität. Nach 18 Monaten stieg der Prozentsatz auf 31 $\%$. Der klinische Zustand der Patienten zum Zeitpunkt der Manifestation der SAB und die Blutmenge auf dem initialen CCT korrelierten in diesem Fall mit der Prognose der Patienten (De Rooij et al. 2013).

In einer multivariaten Analyse mit insgesamt 3567 eingeschlossenen SAB-Patienten, welche in 4 randomisierten kontrollierten Studien rekrutiert wurden, sind folgende Faktoren identifiziert worden, welche mit einer schlechten Prognose nach einer SAB assoziiert sind: höheres Patientenalter, schlechter klinischer Zustand zum Zeitpunkt des Blutungsereignisses (höherer WFNS-Grad), größere Blutmenge in der initialen CCT (höherer Fisher-Grad), Aneurysmalokalisation im Bereich des hinteren Kreislaufes, größeres Aneurysma, das Vorhandensein einer intraventrikulären Blutung oder eines intrazerebralen Hämatoms, höherer systolischer Blutdruck zum Zeitpunkt der Diagnosestellung, bekannte arterielle Hypertonie, eine SAB in der Vorgeschichte, Körpertemperatur von $>38^{\circ} \mathrm{C}$, Auftreten von symptomatischen Vasospasmen oder von zerebralen Infarkten (Kramer et al. 2010, 
Rosengart et al. 2007). Einige dieser Risikofaktoren sind direkt zum Zeitpunkt der Manifestation der SAB vorhanden und sind nicht modifizierbar. Andere Risikofaktoren wie das Auftreten von symptomatischen Vasospasmen und von zerebralen Infarkten werden als potentiell modifizierbare Faktoren angesehen. Daher richtet sich die SAB-Forschung überwiegend auf diese Faktoren mit dem Ziel, die Prognose der Erkrankung zu verbessern. 


\section{$2 \quad$ Material und Methoden}

Dieses Forschungsvorhaben wurde durch die Tierschutzbehörde in Niedersachsen genehmigt (AZ 13/1055). Die Experimente wurden durch die „Deutschen Forschungsgemeinschaft“ (DFG, MI 1894/1-1) sowie die hausinterne Forschungsförderung der UMG mitfinanziert.

\subsection{Tiermodell für die experimentelle Subarachnoidalblutung}

Im Rahmen dieser Studie ist das von Vatter und Güresir modifizierte doppelte Blutinjektionsmodell (double hemorrhage model) bei der Ratte zur Anwendung gekommen (Vatter et al. 2006, Güresir et al. 2015a). Als Versuchstiere dienten männliche SpragueDawley-Ratten (Lieferant Charles River, Deutschland) mit einem Anfangsgewicht von 200 g. Die Versuchstiere wurden 7-10 Tage vor Beginn des Experimentes zur Akklimatisierung geliefert. Die Tiere wurden in einem dafür eingerichteten Raum der Zentralen Tierexperimentellen Einrichtung der Universitätsmedizin Göttingen mit kontrollierter Temperatur $\left(20^{\circ} \mathrm{C}\right)$ und Luftfeuchtigkeit (40-60\%) sowie mit einer automatisch regulierten Raumbeleuchtung zur Aufrechterhaltung des Tag/Nacht-Rhythmus (abwechselnd 12 Stunden hell und 12 Stunden dunkel) untergebracht. Dort konnten die Tiere vor sowie während des Experimentes nach Bedarf essen (Trockenfutter sowie Breifutter) und trinken (Wasser). Zu Beginn des Experimentes wogen die Ratten 250-320 g. Das Körpergewicht der Tiere wurde täglich kontrolliert und dokumentiert. Eine Gewichtsabnahme von mehr als 20 $\%$ des Ausgangsgewichtes innerhalb von 24 Stunden wurde als Abbruchkriterium definiert.

Tabelle 5. Aufbau des Experimentes

\begin{tabular}{|l|l|l|l|l|}
\hline \multicolumn{1}{|c|}{ Tag 1 } & \multicolumn{1}{|c|}{ Tag 2 } & \multicolumn{1}{c|}{ Tag 3 } & \multicolumn{1}{c|}{ Tag 4 } & \multicolumn{1}{c|}{ Tag 5 } \\
\hline $\begin{array}{l}\text { Ligatur der Arteria } \\
\text { carotis commuis }\end{array}$ & Zweite Blutinjektion & Neuroscore & Neuroscore & MRT \\
MRT (Baseline) & & & & $\begin{array}{l}\text { Traskardiale Perfusion } \\
\text { Gehirnentnahme zur } \\
\text { histologischen } \\
\text { Erste Blutinjektion }\end{array}$ \\
MRT & & & & \\
& & & & \\
& & & & \\
\hline
\end{tabular}


Die Induktion der SAB wurde unter Vollnarkose durchgeführt. Die Narkose erfolgte rein intraperitoneal mit einer Mischung aus Medetomidin (Cepetor ${ }^{\circledR} 1 \mathrm{mg} / \mathrm{ml}$ ) und Ketamin (Ketamin ${ }^{\circledR} 10 \%$ Lösung). Die Zusammensetzung der Narkosemittel ist wie folgt: $1 \mathrm{ml}$ Narkosemischung $=0,3 \mathrm{ml}$ Medetomidin $+0,7 \mathrm{ml}$ Ketamin. Die Dosis pro durchgeführte Narkose war 0,1 ml von der Narkosemischung auf $100 \mathrm{~g}$ Körpergewicht der Ratte. Nach der Injektion der Narkose wurden die Ratten in den Käfig zurückgelegt, bis eine tiefe Narkose klinisch (Ausfall Lidreflex und Zwischenzehenreflex) festgestellt werden konnte. Zur Überprüfung der Narkosetiefe wurden die Reflexe vor Beginn der ersten Intervention sowie während der einzelnen Interventionen untersucht und $0,1 \mathrm{ml}$ der Narkosemischung bei Bedarf subkutan zur Aufrechterhaltung der Narkose bis zum Ende der letzten Intervention nachgespritzt. Während der Narkose waren die Tiere in der Lage, selbständig zu atmen, sodass eine maschinelle Beatmung und Intubation der Tiere nicht notwendig gewesen sind. Zum Schutz der Kornea wurde Augengel appliziert.

Die Areale der geplanten Hautinzisionen am Hals und Kopf sowie im Nacken wurden rasiert. Die Körpertemperatur wurde mit Beginn der Narkose bis zum Aufwachen aus der Narkose kontinuierlich gemessen und zwischen $36-38{ }^{\circ} \mathrm{C}$ aufrechterhalten. Hierfür wurde eine Wärmeplatte mit rektaler Temperaturmessung verwendet (Homeothermic Monitor, Harvard Apparatus, Hugo Sachs Elektronik, Deutschland).

Für die Induktion der SAB wurde autologes arterielles Blut $(0,25 \mathrm{ml})$ aus der Schwanzarterie entnommen. Um eine Gerinnung des entnommenen Blutes zu verhindern, wurde 0,05ml Heparin der Spritze beigemengt. Für die Blutentnahme wurden Kanülen mit einer Größe von 27 Gauge (Firma B.Braun Melsungen AG, Melsungen, Deutschland) verwendet. Vor der ersten Blutinjektion wurde zusätzlich die linke Arteria carotis communis ligiert, um die Ischämietoleranz zu senken (Güresir et al. 2015b).

Für die Ligatur der linken Arteria carotis communis wurde die Ratte auf dem Rücken gelagert. Es wurde eine mediane Inzision am Hals durchgeführt. Die Präparation der Arteria carotis communis links erfolgte unter mikrochirurgischen Bedingungen. Durch eine stumpfe und dadurch blutarme Präparation der Halsmuskulatur wurde das Carotisdreieck links eröffnet. Dann wurde ein Wundsperrer eingesetzt und die Arteria carotis communis dargestellt. Die Adventitia wurde unter Schonung des Nervus vagus, welcher der Arterie aufliegt, entfernt. Es wurde eine Ligatur unter Aussparung des N. vagus platziert, um hämodynamische Komplikationen zu vermeiden. Dann wurde die Ligatur zugezogen und mittels dreifacher Knotung fixiert.

Nach Entfernung des Wundsperrers wurde die Haut mit einer Hautnaht verschlossen. Anschließend erfolgte die Blutentnahme für die erste Blutinjektion. Die Ratte wurde hierfür mit dem Kopf in einen stereotaktischen Rahmen eingespannt, sodass der Kopf während der Blutinjektion fixiert war (Abbildung 10). Als nächstes wurde die hintere Schädelgrube für die spätere Blutinjektion über eine Hautinzision am kraniozervikalen Übergang freigelegt. Hierfür wurde eine stumpfe Präparation der Nackenmuskulatur vorgenommen. Dies 
ermöglichte die Darstellung der Membrana atlantooccipitalis nach Einsetzen eines Wundsperrers (Abbildung 11).

Die Membrana atlantooccipitalis wurde unter dem Mikroskop links paramedian inzidiert (1 $\mathrm{mm}$ große Inzision). Dabei wurde die Arachnoidea ebenfalls eröffnet und 0,1 ml Liquor wurden drainiert. Anschließend erfolgte die erste Blutinjektion. Hierbei wurde ein weicher und dünner Katheter (Portexpolythen) mit einem Durchmesser von 0,28 mm verwendet. Nach Zurückziehen des Katheters wurde hämostypisches Material (Tabotamp®, Ethicon, Johnson\&Johnson Medical, Norderstedt, Deutschland) auf die Eröffnung der Membrana atlantooccipitalis gelegt, was ein Austreten des injizierten Blutes verhindern sollte. Der Wundsperrer wurde danach entfernt. Nach der Blutinjektion wurde der Kopf aus dem stereotaktischen Rahmen ausgespannt und die Ratte in einer $20^{\circ}$ Kopftieflage für 15 Minuten platziert, damit es zu einer gleichmäßigen Blutverteilung innerhalb des Subarachnoidalraumens kommt. Währenddessen erfolgte der Hautverschluss mittels Hautnaht (SERALON ${ }^{\circledR}$ 4-0 Polyamid, monofil). Die zweite Blutinjektion erfolgte am Tag 2 auf die gleiche Art und Weise über eine Wiedereröffnung der Operationswunde am kraniozervikalen Übergang.

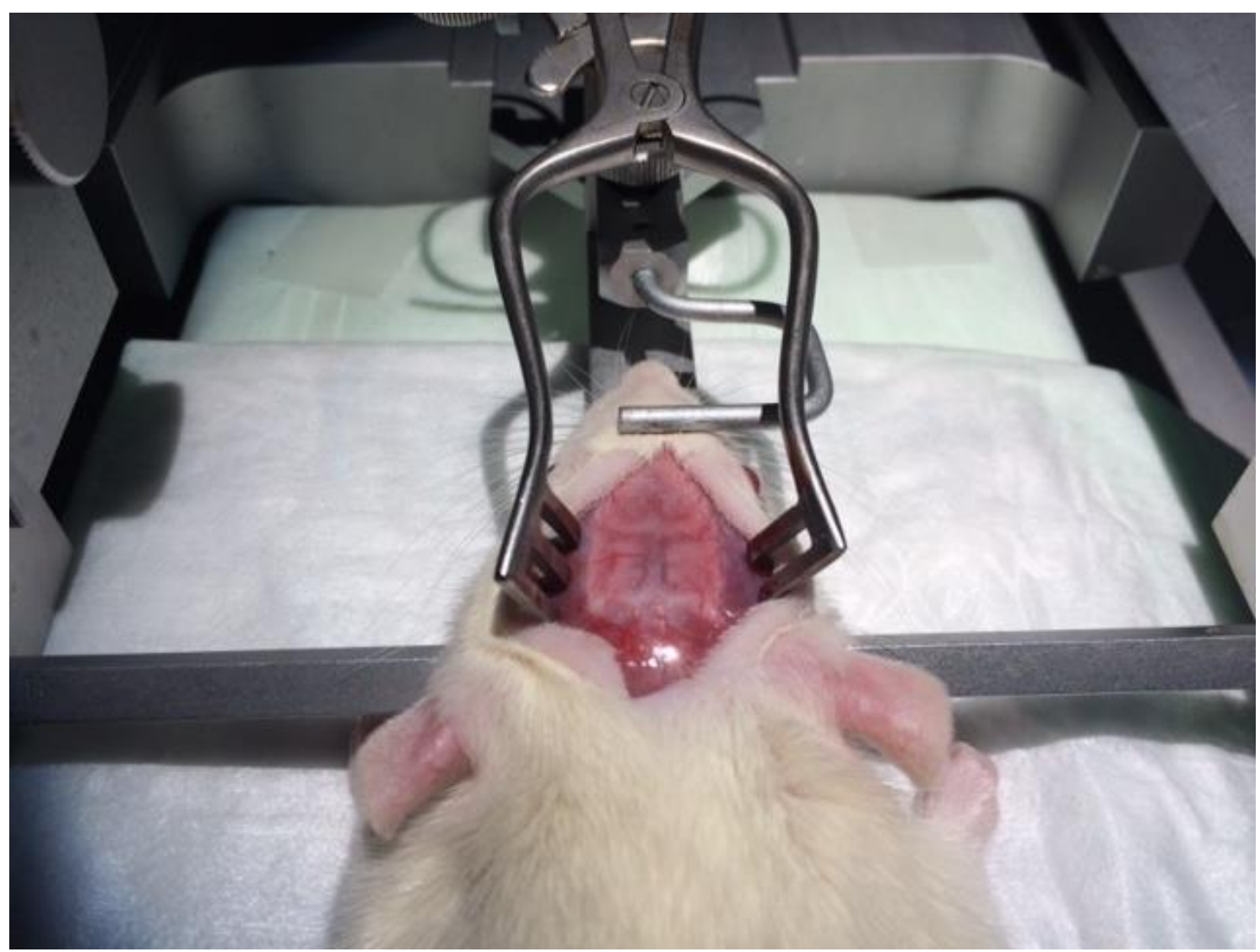

Abbildung 10. Einspannen des Kopfes im stereotaktischen Rahmen 


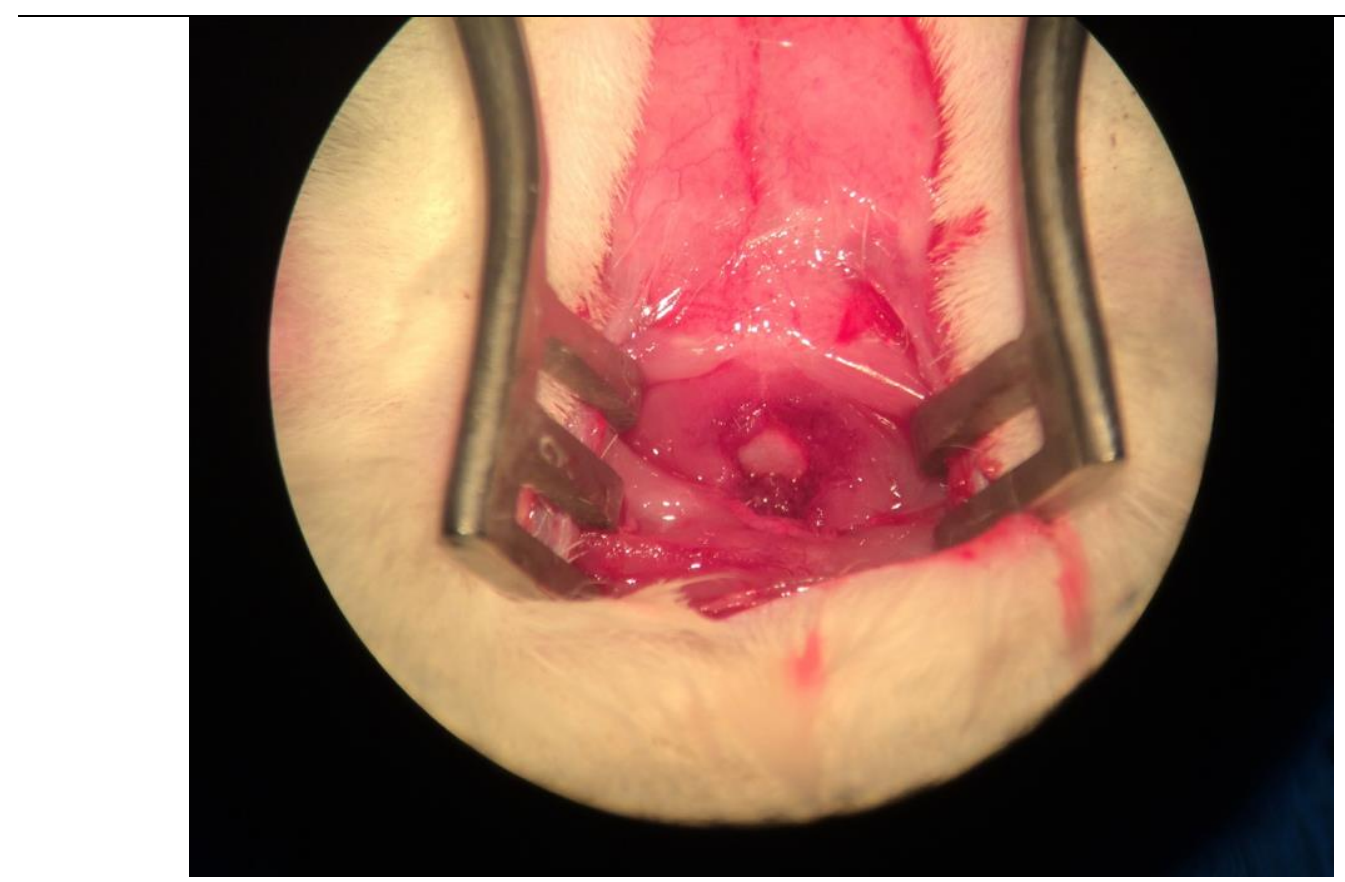

Abbildung 11. Darstellung der Membrana atlantooccipitalis

Am Ende der Intervention wurden die Ratten in einer speziellen Box (Portable Animal Intensive Care Unit, Fa. Thermo Care, Kalifornien, USA) mit konstanter Raumtemperatur und Sauerstoffgehalt zum Aufwachen aus der Narkose gelegt (Abbildung 12). Nachdem die Ratten aus der Narkose aufgewacht waren, wurden sie postoperativ in Einzelkäfige gelegt, um gegenseitige Verletzung zu vermeiden.

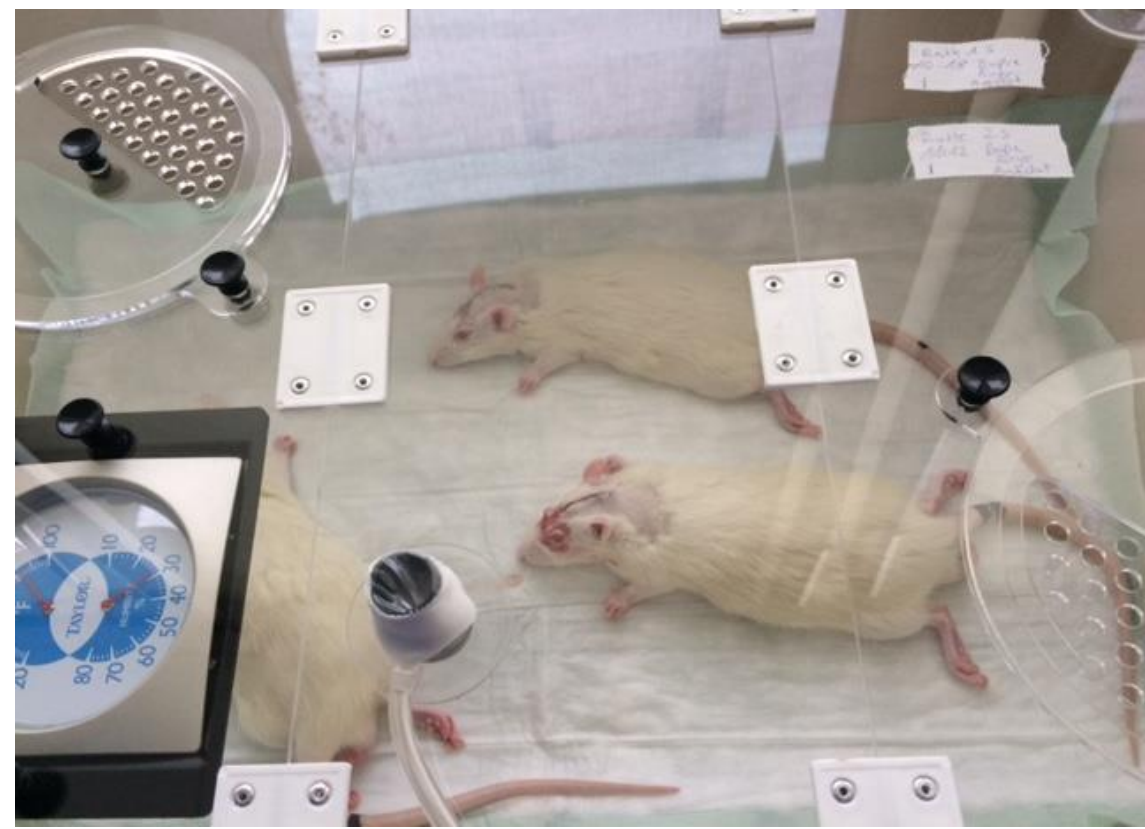

Abbildung 12. Portable Animal Intensive Care Unit 
Während der Dauer des Experimentes und vor allem an den ersten zwei Tagen wurde allen Ratten zur Vermeidung einer Dehydrierung mindestens dreimal täglich Kochsalzlösung (jeweils $5 \mathrm{ml}$ ) subkutan injiziert. Die Schmerzmedikation bestand aus Metamizol $(1,2 \mathrm{mg} / \mathrm{ml}$ Trinkwasser) kontinuierlich bei Bedarf sowie Buprenorphin (Temgesic ${ }^{\circledR}$ 0,03-0,05 $\mu \mathrm{g} / \mathrm{kg}$ bis $0,1 \mu \mathrm{g} / \mathrm{kg}$ Körpergewicht der Ratte) dreimal täglich subkutan injiziert.

In der Sham-Gruppe erfolgten die gleichen operativen Schritte, wie in der SAB-Gruppe, wobei anstatt autologem Blut $0,25 \mathrm{ml}$ Kochsalzlösung an zwei aufeinander folgenden Tagen in die Cisterna cerebellomedullaris injiziert wurde.

Die transkardiale Perfusion am Tag 5 des Experimentes wurde mit phosphatgepufferter Salzlösung (PBS-Lösung) und Paraformaldehyd (PFA 4 \%-Lösung) durchgeführt. Hierfür wurde ein transthorakaler Zugang über eine mediane Hautinzision an der ventralen Thoraxwand mit anschließender Sternotomie durchgeführt. Eine Kanüle wurde in die rechte Kammer vorgeschoben und über eine Perfusionspumpe die PBS-Lösung und PFA-Lösung infundiert. Die Vena cava superior wurde eröffnet, damit das Blut aus dem Kopf durchgespült und das Gehirn fixiert werden kann. Nach der transkardialen Perfusion wurde das Gehirn entnommen, in PFA-Lösung gelegt und in die Neuropathologie zur histologischen Untersuchung verbracht.

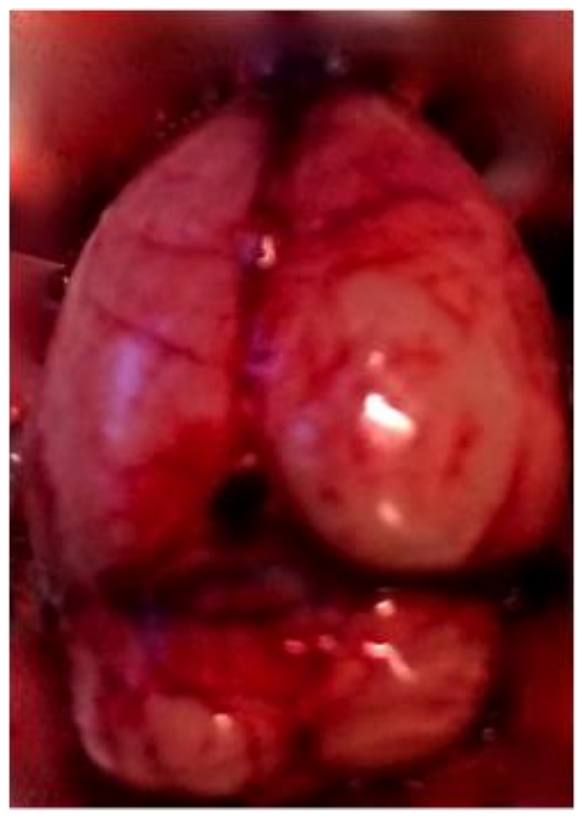

Abbildung 13. Experimentelle SAB nach dem doppelten Injektionsmodell bei der Ratte 


\section{$2.2 \quad$ Bildgebende Untersuchung}

\subsubsection{MRT-Untersuchungen}

Bei allen Ratten wurde eine MRT (Magnetresonanztomographie)-Untersuchung jeweils am Tag 1 vor der ersten Injektion, am Tag 2 nach der zweiten Blutinjektion sowie am Tag 5 am Ende des Experimentes vor der transkardialen Perfusion der Tiere durchgeführt.

Die MRT-Untersuchung erfolgte unter Vollnarkose. Es wurden folgende Sequenzen generiert: T1-, T2-, T2*- sowie eine TOF (time of flight)-Sequenz. Die T1- und T2-Sequenzen wurden erstellt, um die Blutverteilung darzustellen. Die TOF-Sequenz bzw. die MRAngiographie wurden zur Visualisierung der Gefäße angefertigt. Die MRT-Untersuchungen wurden an einem 3 Tesla Gerät durchgeführt (Magnetom TIM Trio, Siemens, Erlangen Deutschland).

Bei der Auswertung der MRT-Untersuchungen diente die erste Untersuchung am Tag 1 vor der ersten Injektion als Ausgangsbefund. Bei der Auswertung der MRT-Untersuchungen am Tag 2 und am Tag 5 erfolgte ein Vergleich mit der Ausgangsuntersuchung zur Sicherstellung einer verlässlichen Interpretation der Ergebnisse am Tag 2 und Tag 5.

Vasospasmen wurden als neu aufgetretene Kaliberschwankungen der basalen Arterien des Circulus arteriosus Willisii definiert. In der MRT-Untersuchung am Tag 2 wurde das Auftreten von frühen Vasospasmen evaluiert. Die MRT-Untersuchung am Tag 5 diente der Diagnose von verspäteten Vasospasmen.

\subsection{Histologische Untersuchungen}

Die fixierten Gehirne wurden in der Neuropathologie eingebettet, geschnitten und gefärbt. Die Hämatoxylin-Eosin (HE)-Färbung wurde für die Darstellung der Blutmenge im Subarachnoidalraum verwendet. Die Verteilung des Blutes auf der einen Seite und die Blutmenge auf der anderen Seite wurden anhand der HE-Färbung analysiert. Hämatoxylin ist ein natürlicher Farbstoff. Das Gewebe wird abhängig von dem pH-Wert gefärbt. Die Strukturen mit niedrigem pH-Wert, insbesondere, Zellkerne werden blau gefärbt. Eosin ist ein synthetischer Farbstoff, welcher die Strukturen mit höherem pH-Wert, beispielsweise Zellplasmaproteine, rot färbt. Die HE-Färbung des Gewebes erfolgte nach einem Standardprotokoll der Abteilung für Neuropathologie. Das Gewebe wurde hierfür zunächst entparaffiniert, woraufhin die Färbung mit Hämalaun erfolgte. Dann wurde das Gewebe mit Aqua destillata und in $1 \% \mathrm{HCl}$-Alkohol zum Differenzieren gespült. Danach erfolgte die Färbung mit Eosin sowie das erneute Abspülen mit Aqua destillata, einer aufsteigenden Alkoholreihe (50\%, 70 \%, $90 \%, 100 \%$ Isopropylalkohol) sowie Xylol. Anschließend wurde das Gewebe in ein Einbettungsmedium (DePex = neurtrale Lösung von Polystyrol und 
Weichmachern in Xylol) eingelegt sowie abschließend geschnitten und unter dem Mikroskop analysiert.

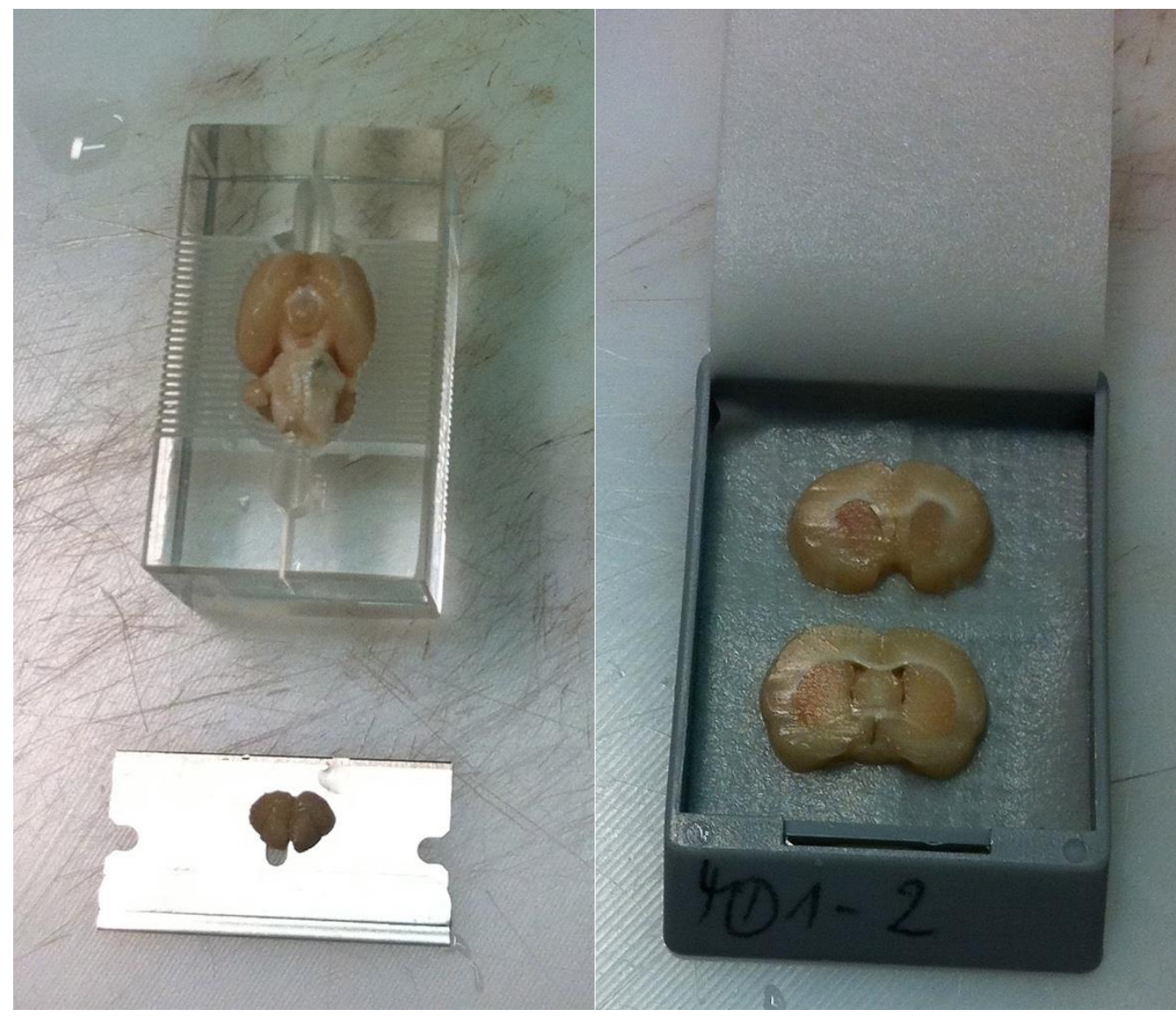

Abbildung 14. Vorbereitung der Schnitte

Bei der Auswertung der histologischen Schnitte wurden die Blutverteilung (Subarachnoidalraum sowie Ventrikelsystem) und die Blutmenge evaluiert. Die Auswertung der Blutmenge erfolgte semiquantitativ, wobei eine Einteilung in geringgradig, mittelgradig und hochgradig vorgenommen wurde. Andererseits wurde die Reaktion der Meningen (Hirnhäute) auf die Blutungsausdehnung analysiert.

\subsection{Klinische Untersuchungen}

Die Tiere wurden täglich mit Hilfe eines neurologischen Scores untersucht, welcher die motorische Aktivität beurteilt (Jeon et al. 2009). Weiterhin wurde der Stellreflex überprüft. Dieser ist normal, wenn das Tier die kontralaterale Vorderpfote ausstrecken kann, wenn es am Schwanz knapp über dem Boden angehoben wird. Wenn die Vorderpfote nicht ausgestreckt wird und es zusätzlich zu einer Lateralflexion des Körpers zur kontralateralen Seite kommt, ist der Stellreflex pathologisch. Für eine normale motorische Aktivität wurden 
0 Punkte vergeben, für einen pathologischen Stellreflex wurde 1 Punkt vergeben, für Kreisen zur kontralateralen Seite 2 Punkte, für Fallen auf die kontralaterale Seite 3 Punkte, und für fehlende motorische Aktivität wurden 4 Punkte vergeben.

\subsection{Statistische Methoden}

Für die Berechnung der Häufigkeit der einzelnen SAB-Grade wurden deskriptive statistische Methoden verwendet. 


\section{$3 \quad$ Ergebnisse}

\subsection{Induktion der SAB mit dem double hemorrbage model}

Es wurden insgesamt 103 Ratten operiert. Bei 98 Ratten wurde eine SAB induziert, und 5 Ratten unterliefen eine Sham-Operation. Die Gesamtmortalität in der SAB-Gruppe lag bei $52 \%$. Die Ratten verstarben direkt nach der Blutinjektion, entweder am ersten oder zweiten Tag. Das komplette Experiment überlebten 47 Ratten in der SAB-Gruppe, welche in die statistische Auswertung eingeschlossen werden konnten. In der Sham-Gruppe hatten alle 5 Ratten bis zum Ende des Experimentes überlebt.

\subsection{Definition der Schweregrade der SAB}

Die Definition der Schweregrade der SAB richtete sich alleine nach der Blutmenge und nach der Blutverteilung. In der SAB-Gruppe konnte bei 45/47 (96\%) Ratten eine Blutung im Subarachnoidalraum festgestellt werden. Bei zwei Ratten der SAB-Gruppe war in den histologischen Schnitten keine Blutung zu sehen. In der Sham-Gruppe war bei keinem Tier eine Blutung sichtbar.

Grad-1-Blutung: Wenn keine intrakranielle (weder im Subarachnoidalraum noch in den Hirnventrikeln oder im Hirnparenchym) Blutung zu sehen gewesen ist, wurde das als Grad1 klassifiziert. Diese Klassifikation entfiel auf alle Ratten der Sham-Gruppe und auf zwei Ratten der SAB-Gruppe. Die Abbildungen 15-17 zeigen Beispiele der Grad-1-Blutung.

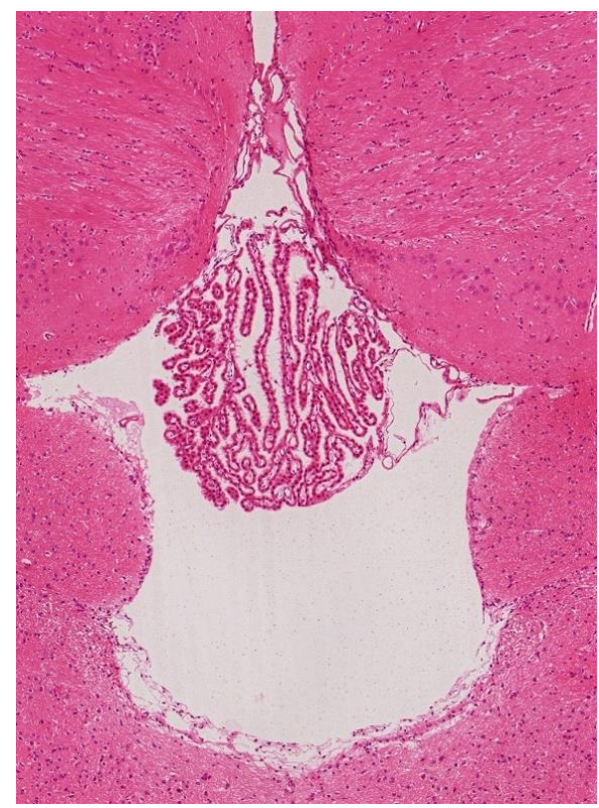

Abbildung 15. Grad-1-Blutung (Darstellung des Ventrikels mit Plexus choroideus, ohne sichtbare intraventrikuläre Blutung) 


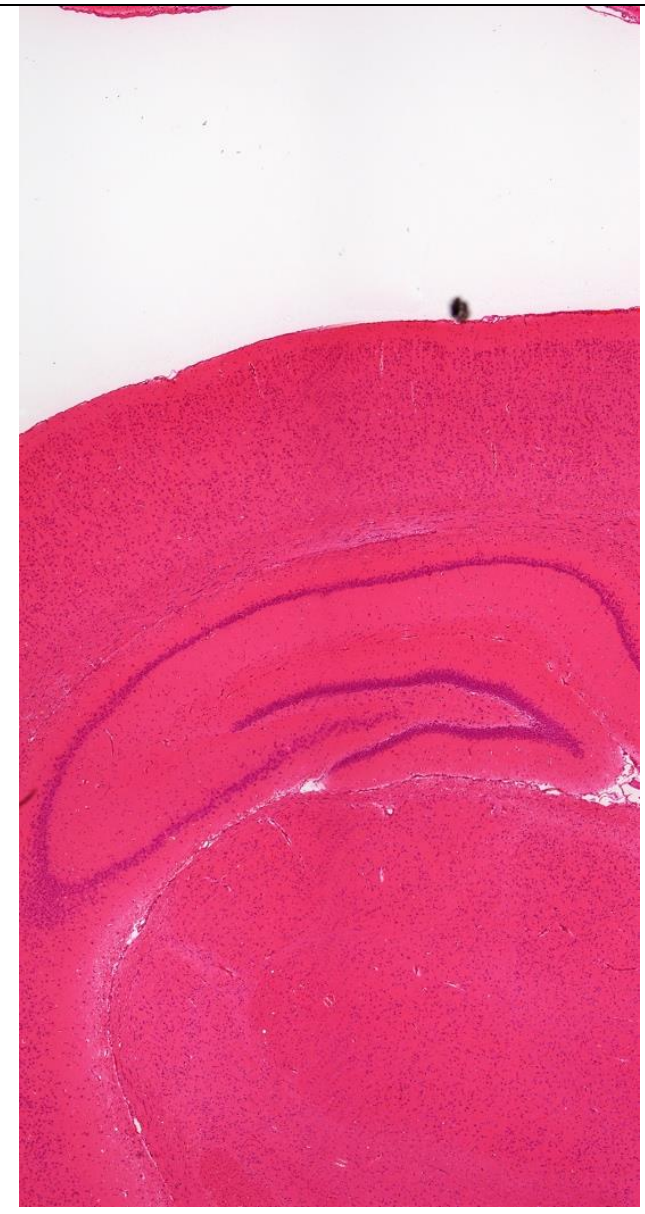

Abbildung 16. Grad-1-Blutung (Darstellung eines Anteils der linken Hemisphäre ohne sichtbare Blutung an der Hirnoberfläche)

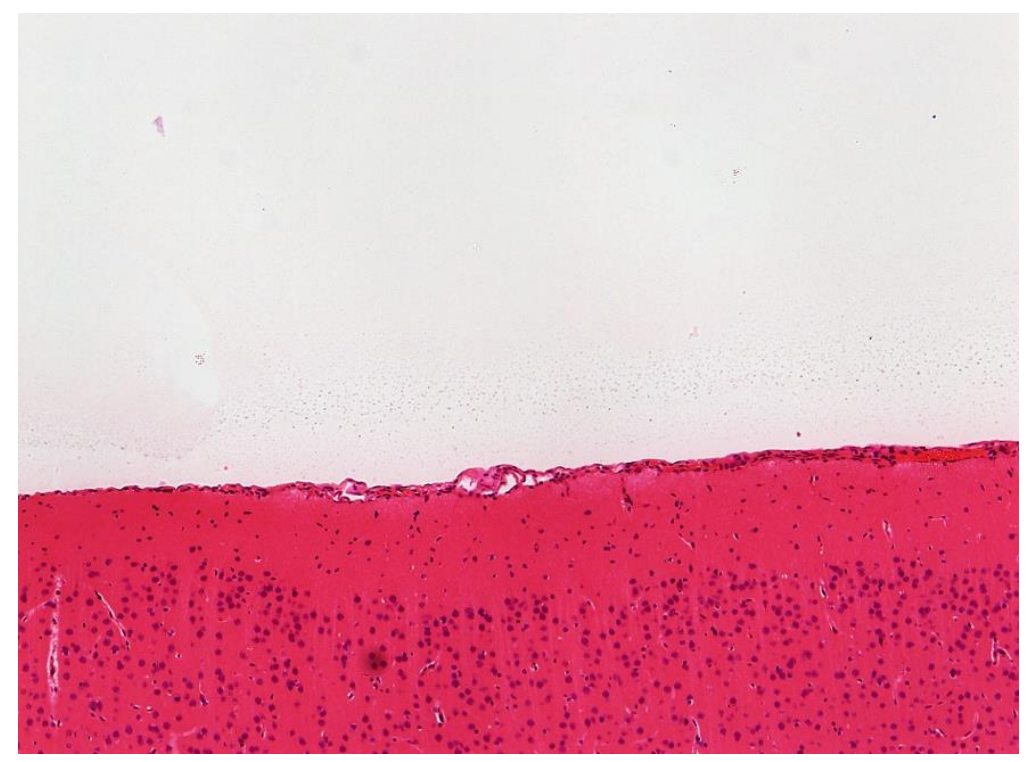

Abbildung 17. Grad-1-Blutung (Darstellung eines Anteils der Hirnoberfläche der linken Hemisphäre mit einer starken Vergrößerung ohne sichtbare Blutung) 
Grad-2-Blutung: Im Fall einer geringen lokalen oder diffusen Blutung wurde ein Grad-2 vergeben. Der Grad-2 ist definiert als eine geringgradige Blutung in den Subarachnoidalraum bei fehlender Blutung innerhalb des Ventrikelsystems. Die Abbildungen 18-24 zeigen Beispiele einer Grad-2-Blutung.

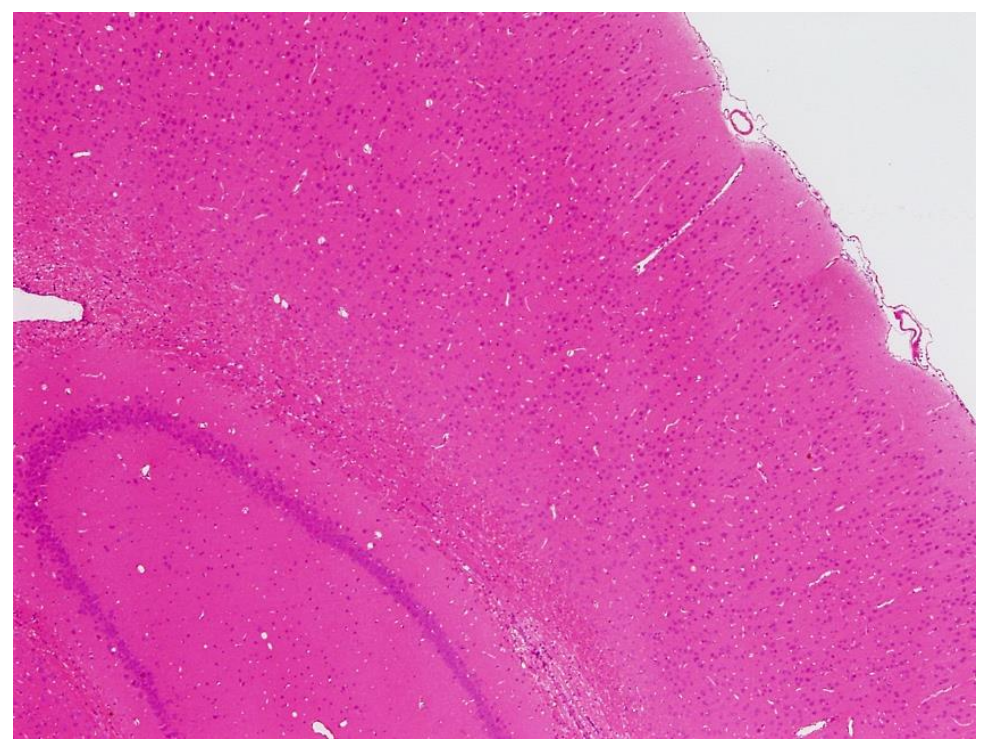

Abbildung 18. Grad-2-Blutung (Darstellung der Hirnoberfläche mit sichtbarer Blutung in den Subarachnoidalraum an der Gehirnoberfläche)

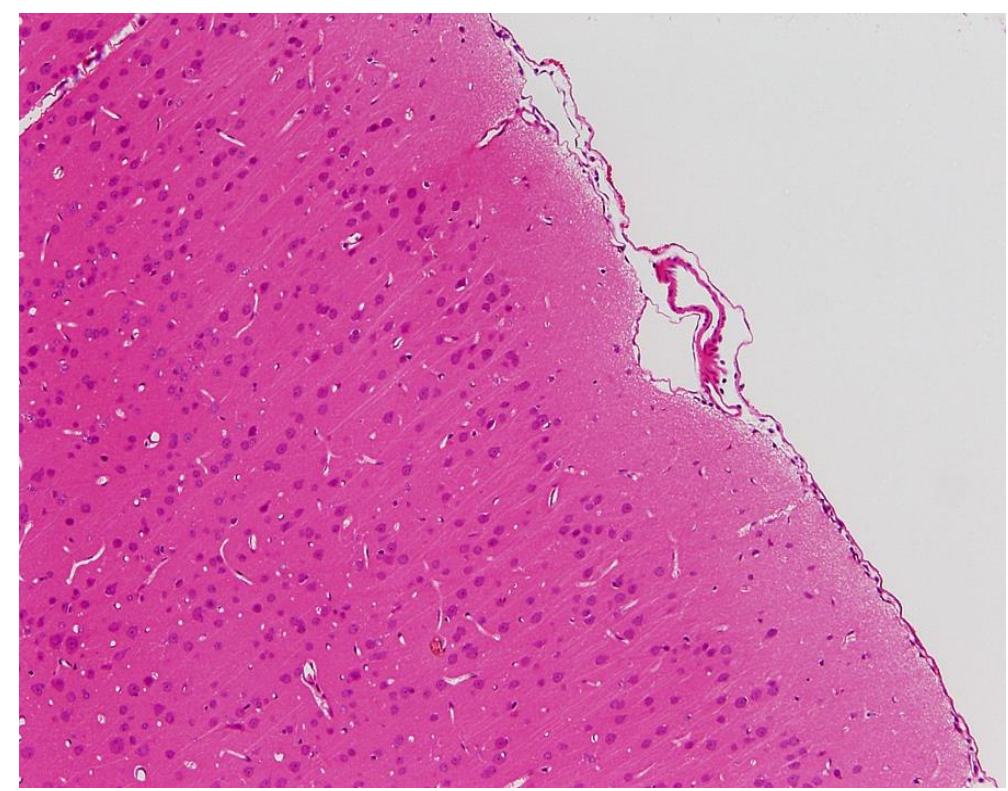

Abbildung 19. Grad-2-Blutung (stärkerer Vergrößerung mit Darstellung des Blutes an der Hirnoberfläche) 


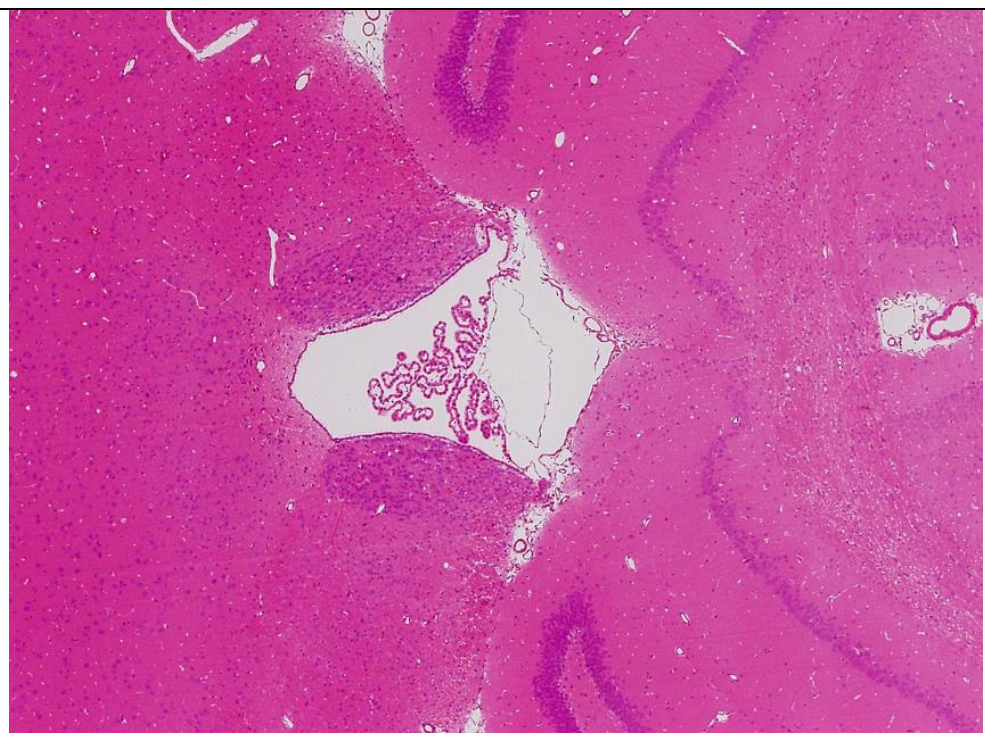

Abbildung 20. Grad-2-Blutung (Darstellung des Ventrikels ohne sichtbare intraventrikuläre Blutung)

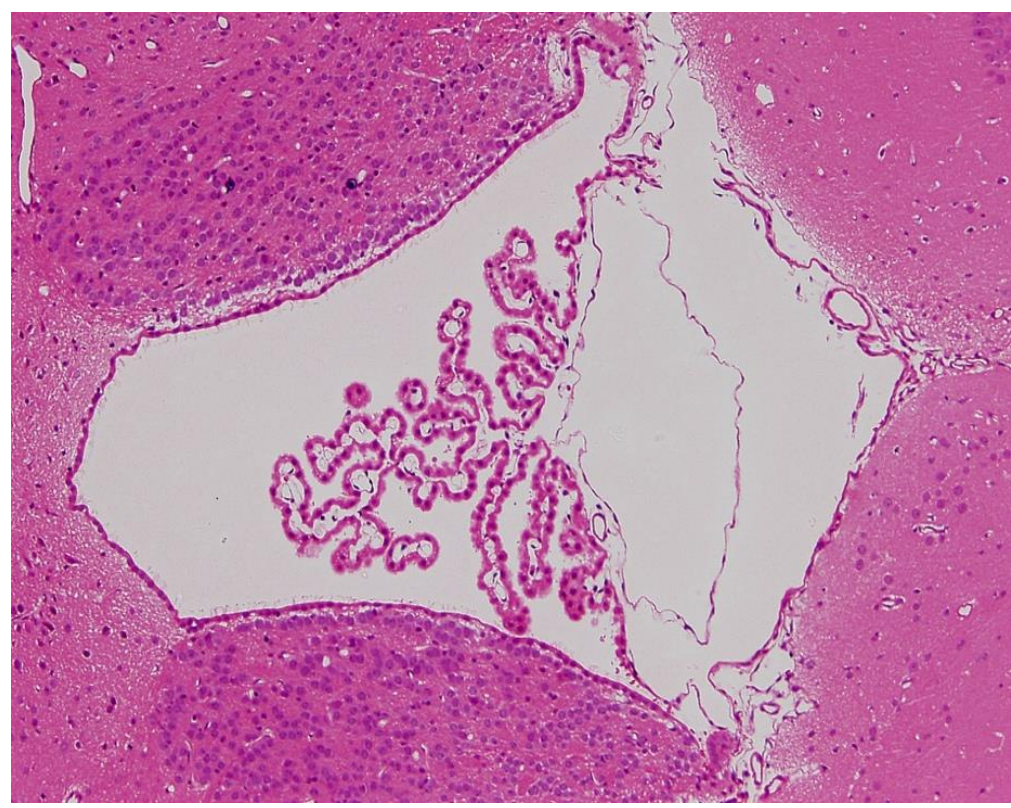

Abbildung 21. Grad-2-Blutung (stärkere Vergrößerung mit Darstellung des Plexus chororideus und des Ependyms ohne sichtbare intraventrikuläre Blutung) 


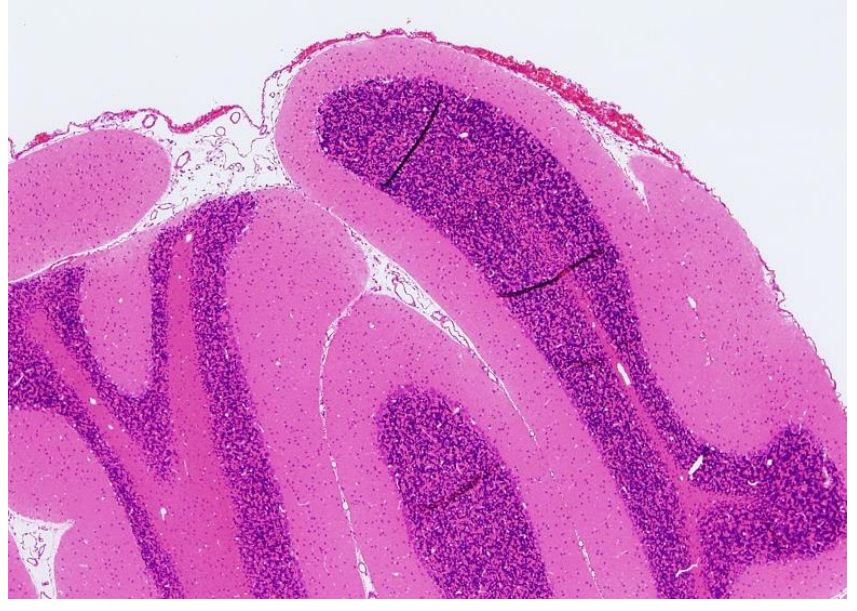

Abbildung 22. Grad-2-Blutung (ausgeprägte diffus verteile Blutung an der Hirnoberfläche)

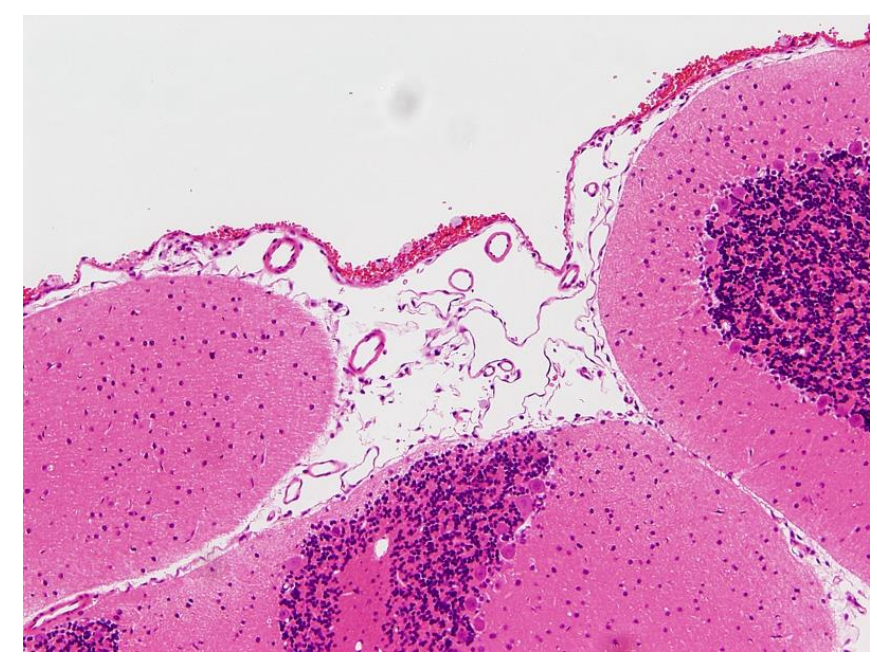

Abbildung 23. Grad-2-Blutung (stärkere Vergrößerung mit Darstellung der Blutverteilung innerhalb des Subarachnoidalraumes)

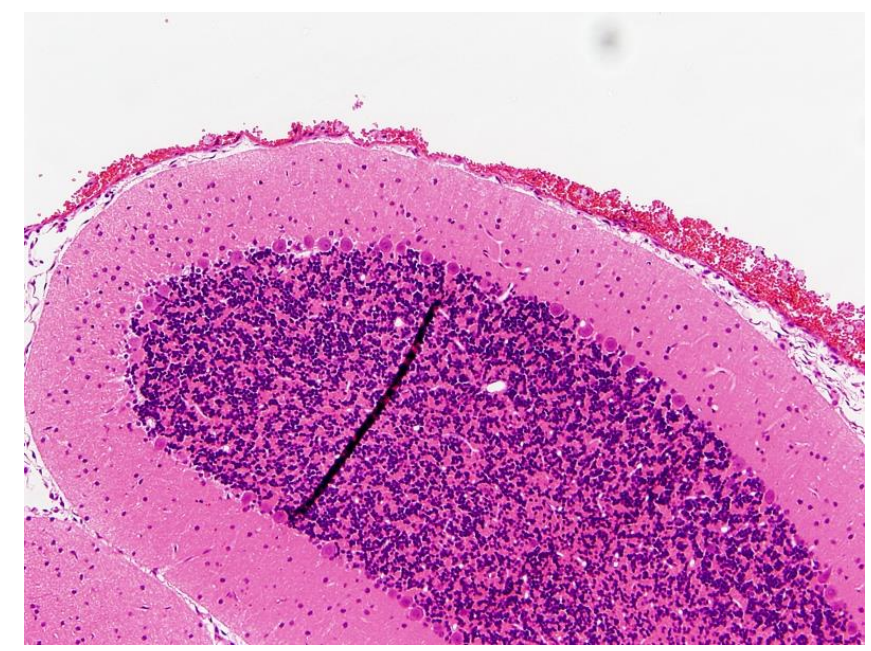

Abbildung 24. Grad-2-Blutung (stärkere Vergrößerung mit Darstellung der Blutmenge an der Hirnoberfläche) 
Grad-3-Blutung: Ein größeres Blutvolumen, welches innerhalb des Subarachnoidalraumes lokal oder diffus verteilt ist, wurde als Grad-3-Blutung definiert. In den Abbildungen 25-28 ist ein Beispiel für eine Grad-3-Blutung dargestellt.

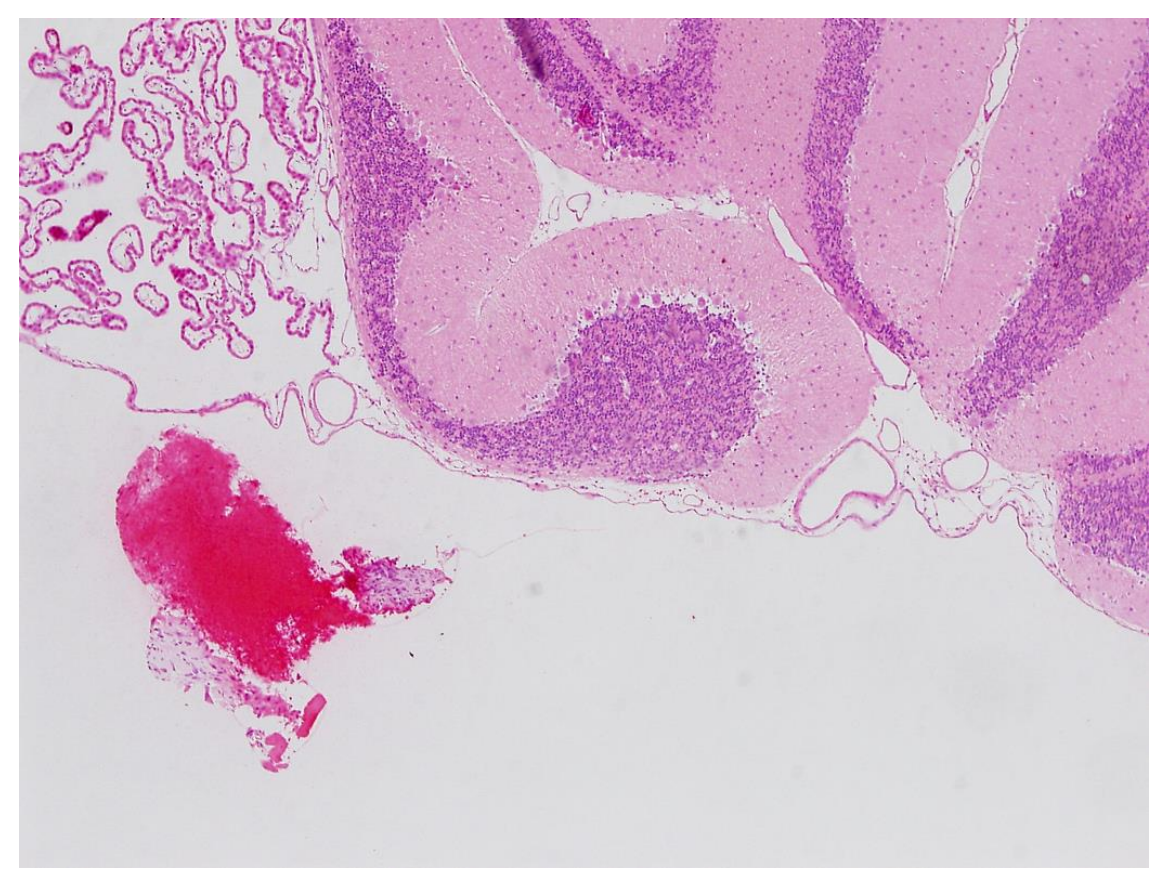

Abbildung 25. Grad-3-Blutung (Darstellung eines lokalen Blutclots im Subarachnoidalraum)

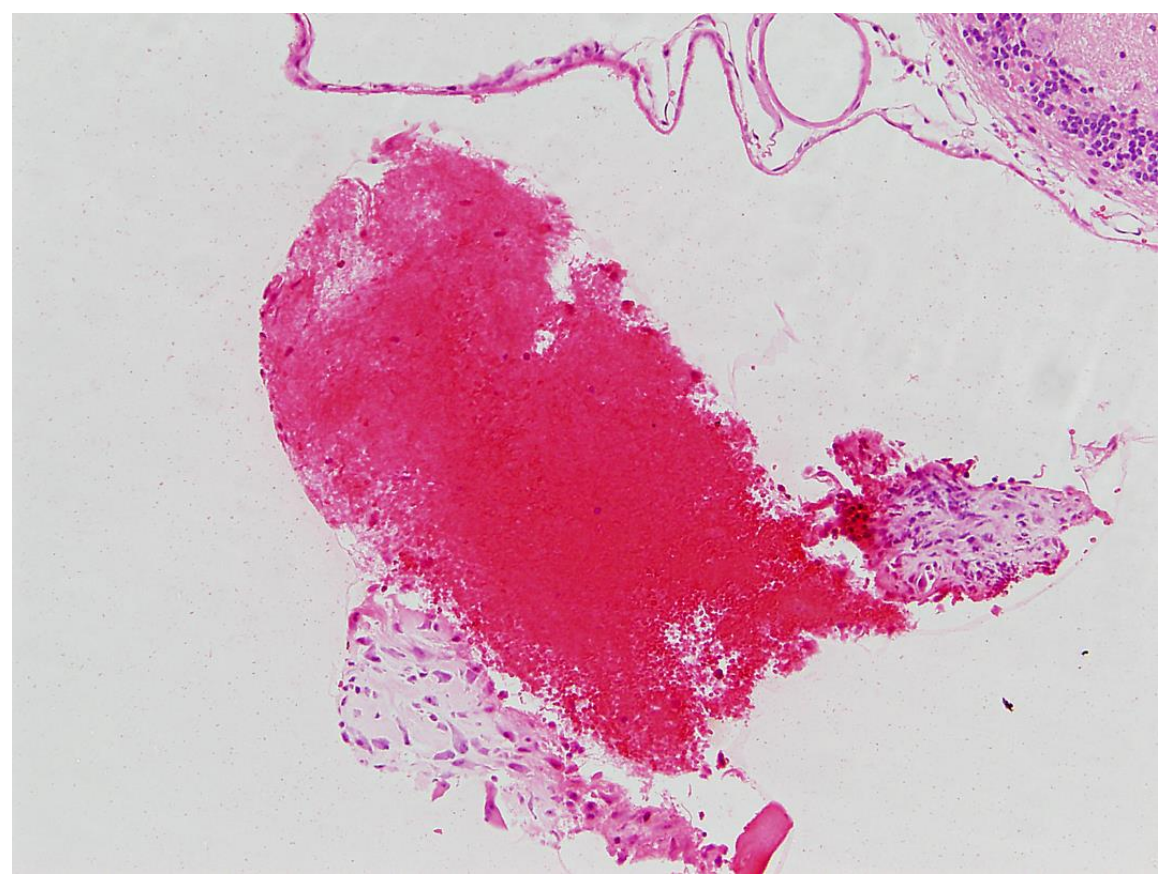

Abbildung 26. Grad-3-Blutung (stärkere Vergrößerung mit Darstellung des Blutclots) 


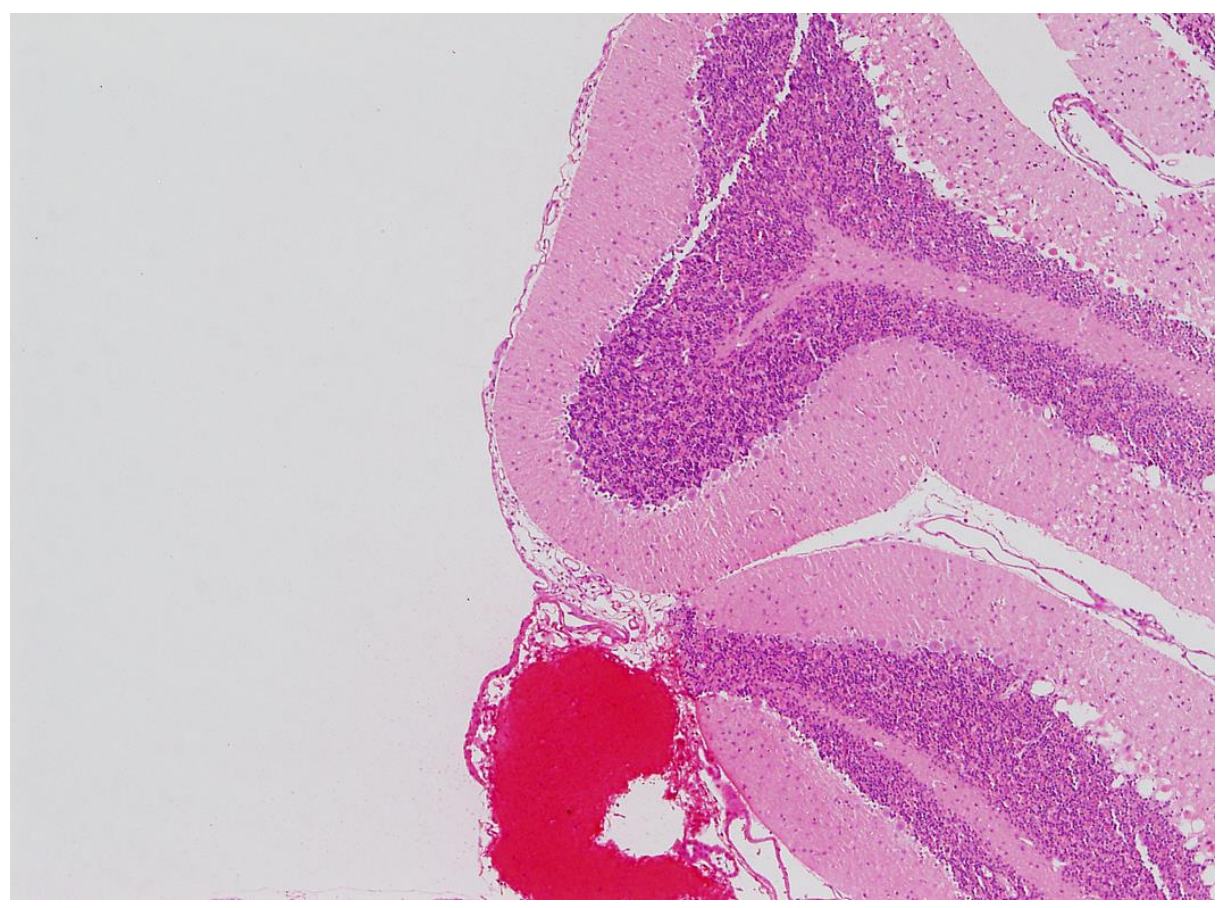

Abbildung 27. Grad-3-Blutung (Darstellung einer ausgeprägten lokalen Blutung im Subarachnoidalraum)

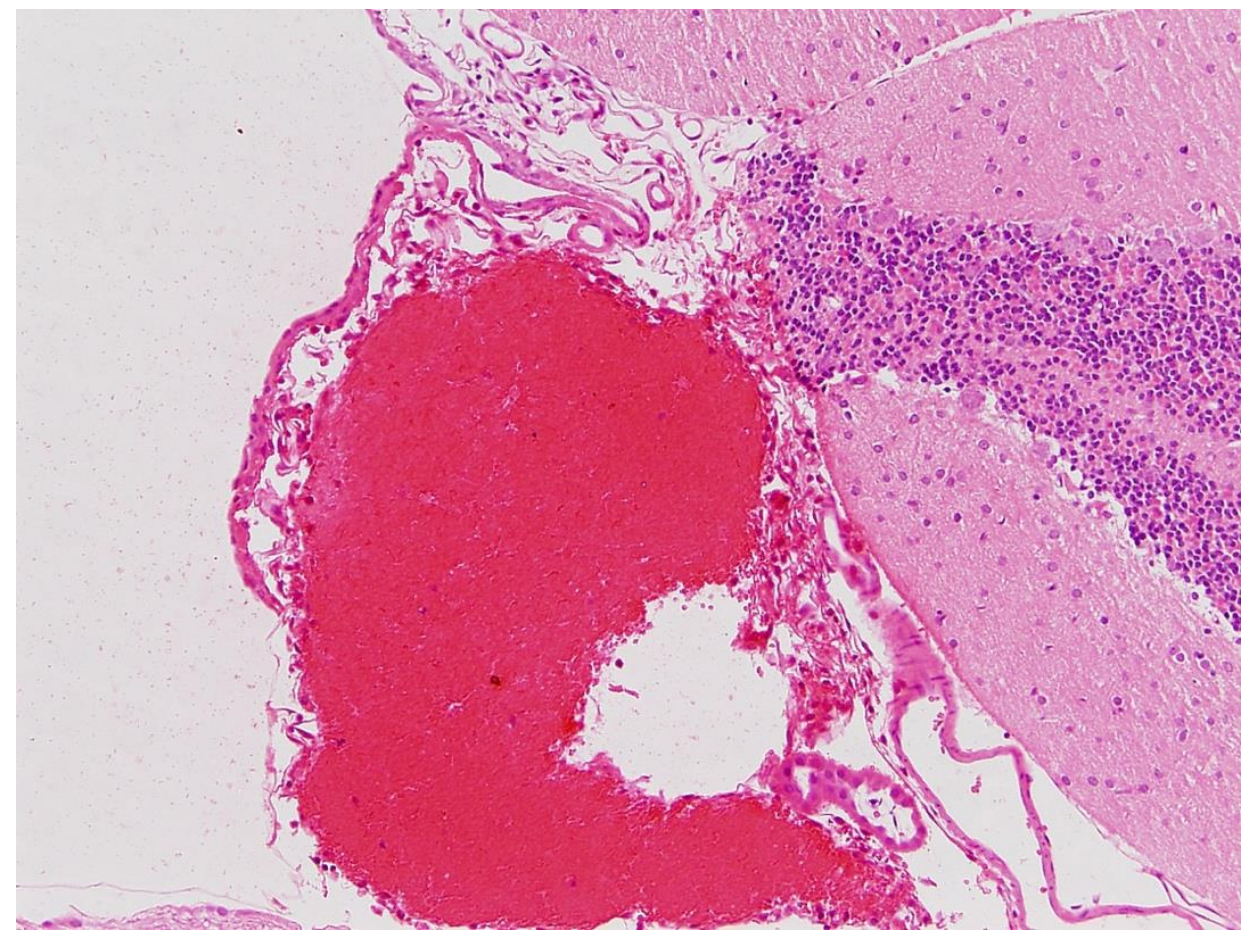

Abbildung 28. Grad-3-Blutung (stärkere Vergrößerung mit Fokus auf die Blutung im Subarachnoidalraum) 
Grad-4-Blutung: Eine Grad-4-Blutung lag vor, wenn sich die Blutung zusätzlich in die Hirnventrikel ausgedehnt hat. Die Abbildungen 29-30 zeigen Beispiele einer Grad-4Blutung.

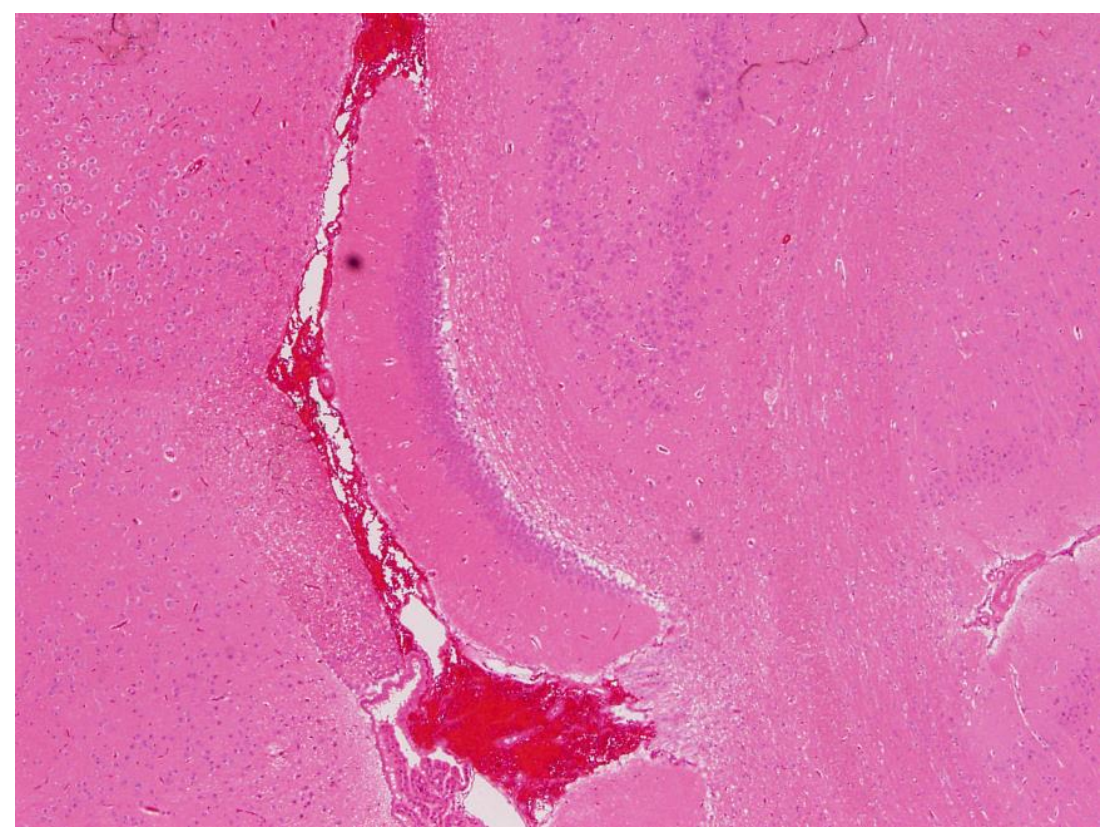

Abbildung 29. Grad-4-Blutung (Darstellung des Ventrikels mit ausgeprägter intraventrikulärer Blutung)

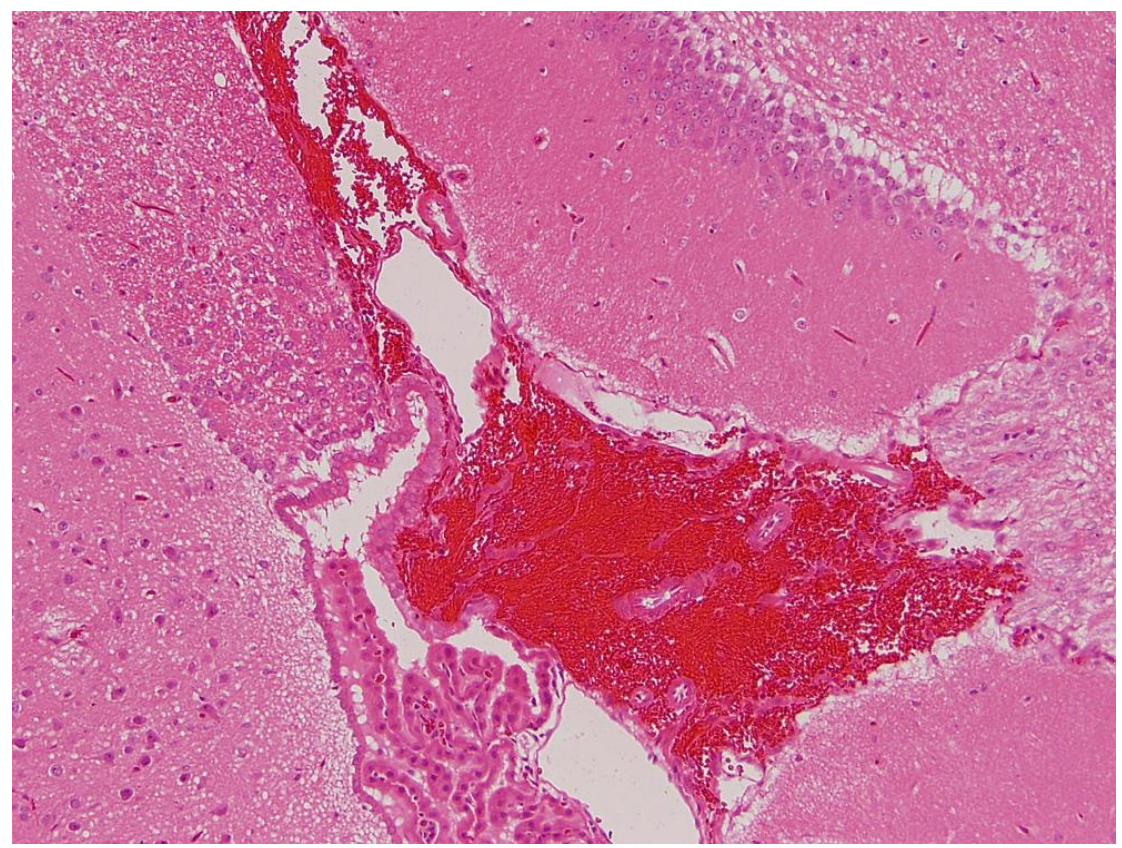

Abbildung 30. Grad-4-Blutung (stärkere Vergrößerung mit Fokus auf die Ventrikelblutung) 


\subsection{Reaktion der Meningen auf die Blutung}

Bei allen Ratten in der SAB-Gruppe konnte eine Reaktion der Meningen (Hirnhäute) festgestellt werden. Die Meningen wiesen eine Zellproliferation auf, die unterschiedlich ausgeprägt war, sodass eine semiquantitative Einteilung in geringgradig und hochgradig vorgenommen werden konnte. In 25/47 (54\%) Ratten der SAB-Gruppe konnte eine geringgradige Reaktion der Meningen dargestellt werden. Bei 21/47 (45\%) Ratten der SABGruppe war eine hochgradige Reaktion der Meningen zu sehen und bei einer SAB-Ratte war keine Reaktion der Meningen vorhanden. Die zwei SAB-Ratten mit Grad-1-Blutung zeigten eine geringgradige Reaktion der Meningen. In der Sham-Gruppe hingegen wurde keine Reaktion der Meningen beobachtet. Die Abbildungen 31 und 32 zeigen Beispiele für keine Reaktion der Meningen beziehunsgweise für eine Meningenreaktion durch die Blutung.

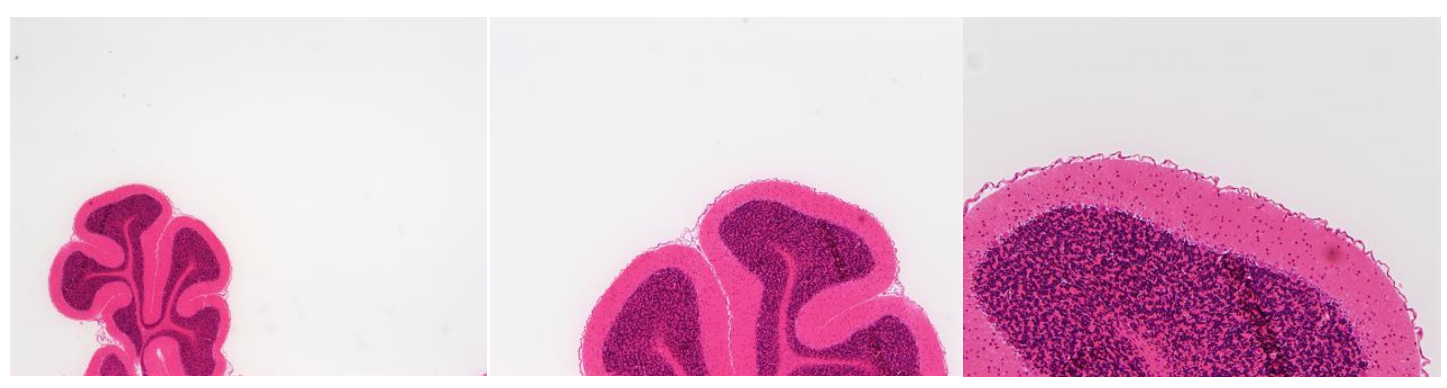

Abbildung 31. Darstellung der Gehirnoberfläche mit dem Subarachnoidalraum ohne sichtbare Reaktion der Meningen mit zunehmender Bildvergrößerung von links nach rechts (2-fach, 4 -fach und 10-fach)

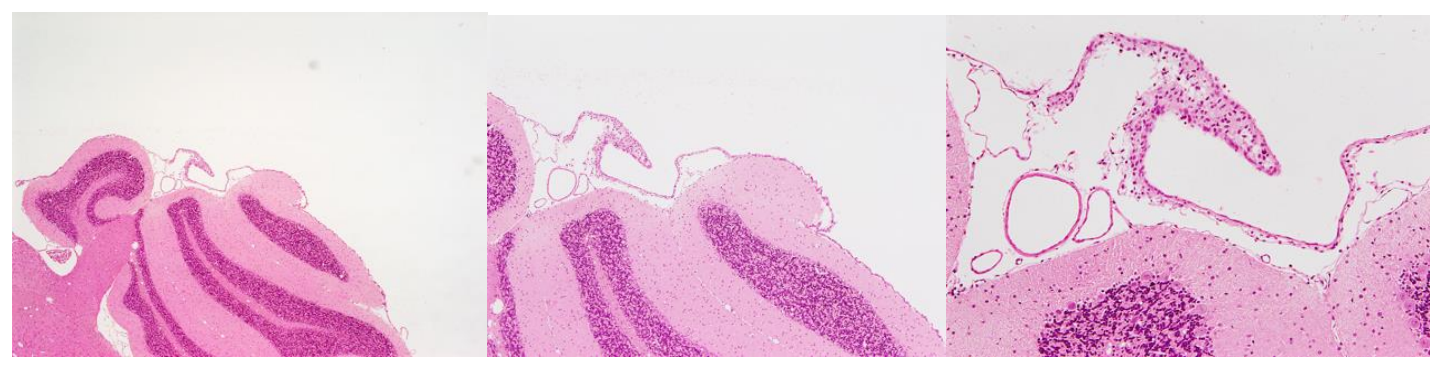

Abbildung 32. Darstellung der Gehirnoberfläche mit dem Subarachnoidalraum mit hochgradiger Reaktion der Meningen mit zunehmender Bildvergrößerung von links nach rechts (2-fach, 4-fach und 10-fach) 


\subsection{Häufigkeit der einzelnen SAB-Grade}

Bei 2/47 (4,3\%) Ratten aus der SAB-Gruppe war keine Blutung nachweisbar, sodass Grad 1 vergeben wurde. Eine Grad 2 Blutung konnte bei 16/47 (3\%) der Ratten in der SABGruppe festgestellt werden. In der SAB-Gruppe war die Grad 3 Blutung in 11/47 (23,4 \%) Tieren vertreten. Der Großteil der Versuchstiere in der SAB-Gruppe, nämlich 18/47 (38,3 $\%$ ) hatten eine Grad 4 Blutung. In der Abbildung 33 ist die Verteilung der einzelnen SABGrade graphisch dargestellt.

In 29/47 (61,7 \%) Ratten in der SAB-Gruppe zeigte ein höherer SAB-Grad ebenfalls eine hochgradige Reaktion der Meningen.

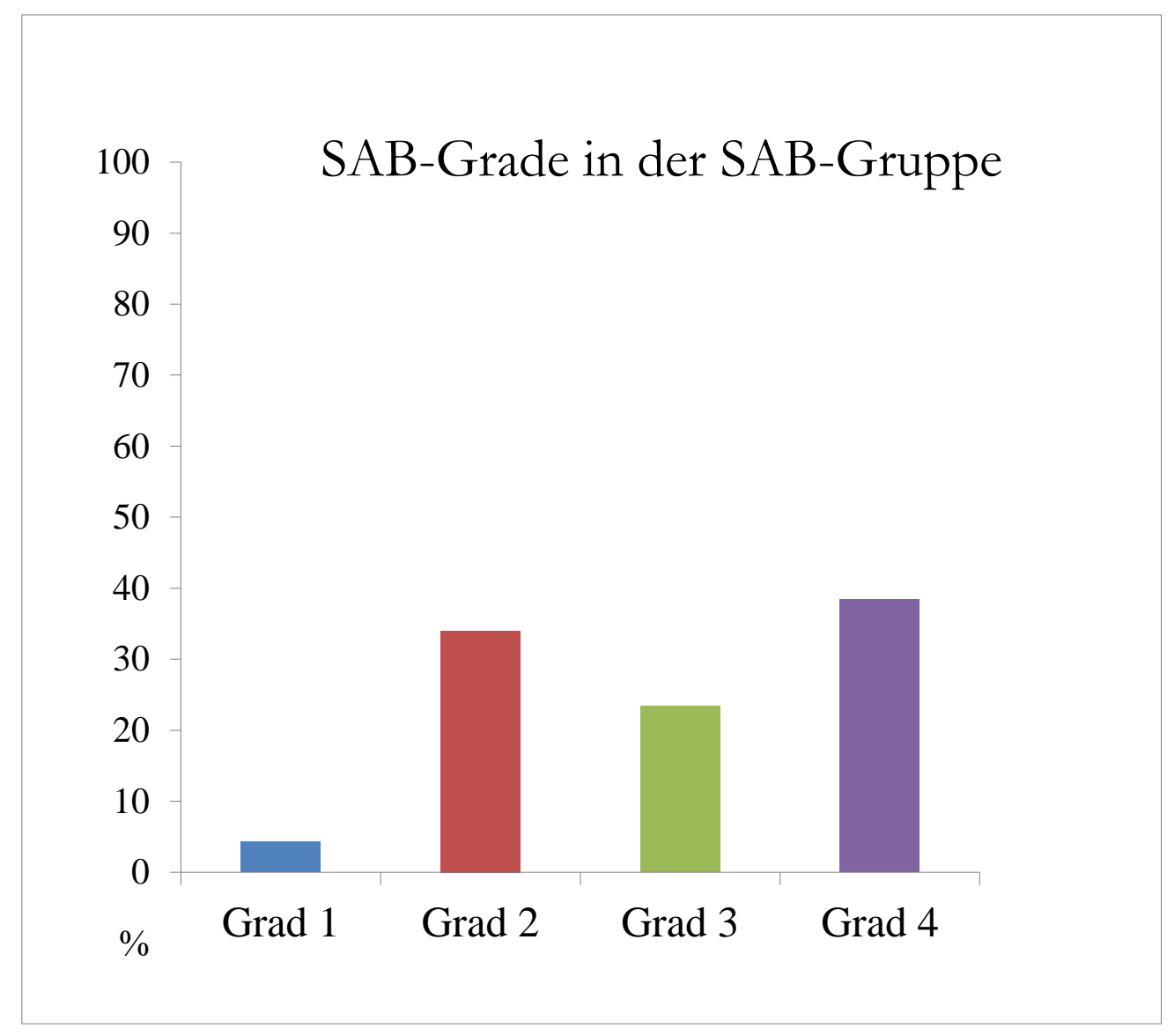

Abbildung 33. Verteilung der einzelnen SAB-Grade in der SAB-Gruppe 


\subsection{Klinischer und neurologischer Status der Ratten}

Am Tag 3 konnte man bei insgesamt 15/47 (32\%) Ratten in der SAB-Gruppe eine klinische Verschlechterung feststellen. Bei 7 Ratten war ein eingeschränkter Stellreflex feststellbar, 6 Ratten zeigten ein Kreisen zur kontralateralen Seite und 2 Ratten haben ein Fallen zur kontralateralen Seite aufgewiesen. Am Tag 4 konnte man bereits eine klinische Verbesserung feststellen, wobei 6/47 (13\%) Ratten in der SAB-Gruppe eine Auffälligkeit gezeigt haben. Bei 2 Ratten war ein eingeschränkter Stellreflex vorhanden und 4 Ratten haben ein Kreisen zur kontralateralen Seite gezeigt. Am Tag 5 haben nur 3/47 (7\%) einen eingeschränkten Stellreflex geboten. In der Sham-Gruppe wurde bei allen 5 Ratten eine normale motorische Aktivität beobachtet.

Am Tag 2 hatten 15/47 (32\%) Ratten in der SAB-Gruppe eine Gewichtsreduktion um $5 \%$ und 2/47 (5\%) Ratten hatten eine Gewichtsreduktion um $10 \%$ bezogen auf das präoperative Körpergewicht. In der Sham-Gruppe hatte 1/5 (20\%) Ratte eine Gewichtsreduktion von $5 \%$.

Am Tag 3 hatten 18/47 (39\%) in der SAB-Gruppe und 2/5 (40\%) Ratten in der ShamGruppe eine Gewichtsreduktion um $5 \%$ bezogen auf das präoperative Gewicht. In 19/47 (41\%) Ratten in der SAB-Gruppe war eine Gewichtsreduktion um $10 \%$ feststellbar.

Am Tag 4 war in 27/47 (58 \%) der Ratten in der SAB-Gruppe und in 4/5 (80 \%) Ratten in der Sham-Gruppe eine Gewichtsreduktion um $5 \%$ feststellbar. Eine Gewichtsreduktion um 10\% wurde bei 15/47 (32\%) der Ratten in der SAB-Gruppe festgestellt. In der ShamGruppe hatte keine der 5 Ratten eine Gewichtsreduktion um $10 \%$ erreicht.

Am Tag 5 hatten 28/47 (60\%) Ratten in der SAB-Gruppe und 3/5 (65\%) Ratten in der Sham-Gruppe eine Gewichtsreduktion um 5\%. Eine Gewichtsreduktion um $10 \%$ hatten 13/47 (28\%) Ratten in der SAB-Gruppe und 1/5 (20\%) Ratten in der Sham-Gruppe. 


\subsection{Ergebnisse der MRT-Untersuchungen}

Die MRT-Untersuchungen konnten bei allen 52 Ratten, welche das komplette Experiment überlebt hatten und in die Auswertungen dieser Arbeit eingeschlossen wurden, durchgeführt werden. Die Dauer jeder einzelnen Untersuchung lag bei 45 Minuten. Die MRTUntersuchung hatte nicht die notwendige Auflösung, um die Blutung im Subarachnoidalraum suffizient darzustellen.

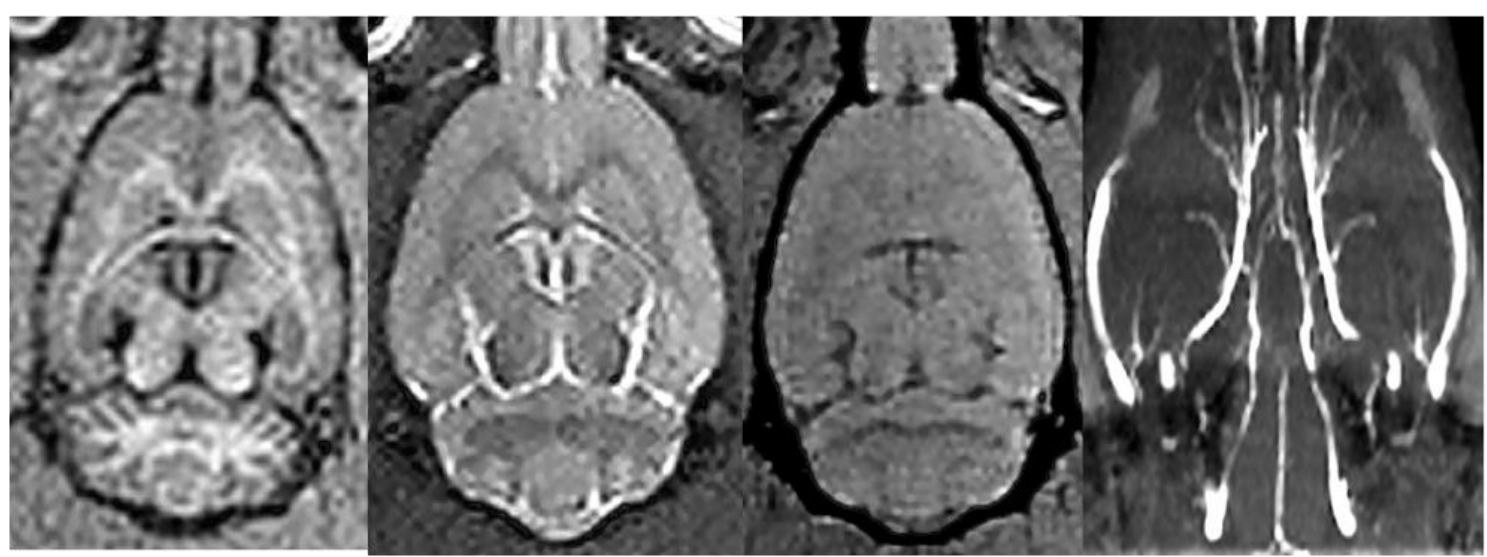

Abbildung 34. Sequenzen der MRT-Untersuchungen 


\section{$4 \quad$ Diskussion}

SAB-Tiermodelle tragen wesentlich zum besseren Verständnis der pathophysiologischen Vorgänge nach einer SAB bei. In der klinischen Praxis sind Klassifikationen zur Beurteilung des Schweregrades einer SAB sinnvoll, um Risiken für mögliche Komplikationen nach dem Blutungsereignis einschätzen zu können und sukzessive vorbeugende, diagnostische und therapeutische Maßnahmen einleiten zu können. Damit die Ergebnisse der tierexperimentellen Forschung verlässlicher auf die klinische Praxis übertragen werden können, ist die Etablierung von Klassifikationssystemen für die experimentelle SAB ebenfalls von besonderer Bedeutung. Bisher wurden solche Klassifikationssysteme für die experimentelle SAB nicht etabliert. Bei dieser Arbeit haben wir uns zum Ziel gesetzt, ein Grading-System zur Beurteilung des Schweregrades der experimentellen SAB durch das doppelte Injektionsmodell bei der Ratte zu erarbeiten. Mit Hilfe der experimentellen Ergebnisse konnten wir, basierend auf einem histologischen Grading-System, analog zu der am häufigsten in der klinischen Praxis eingesetzten Fisher-Skala ein solches System definieren.

\subsection{Diskussion des Tierversuchsmodells}

Das erste SAB-Tiermodell bei der Ratte wurde im Jahr 1979 beschrieben (Barry et al. 1979). Das Modell erfordert eine Eröffnung des Schädels (Kraniotomie) zur konsekutiven Punktion der Arteria basilaris. Im Jahr 1995 wurde ein SAB-Modell bei der Ratte ohne die Notwendigkeit einer Kraniotomie vorgestellt (Bederson et al. 1995). Hierbei wird die Blutung über eine Gefäßperforation im Bereich der intrakraniellen Gefäße induziert.

Insgesamt lassen sich die SAB-Tiermodelle in zwei Hauptgruppen einteilen: die Perforationsmodelle (Prunell et al. 2004, Sehba 2014) und die Injektionsmodelle ( $\underline{\text { Vatter et }}$ al. 2006, Güresir et al. 2015a, Gules et al. 2002, van den Bergh et al. 2005, Raslan et al. 2012). Beim Perforationsmodell (auch Monofilamentmodell genannt) wird ein spitzes Fadenfilament über einen ventralen Zugang am Hals in die Arteria carotis interna vorgeschoben und im Bereich der Aufteilung der Arteria carotis interna (der sogenannte Carotis-Terminus) eine Perforation durchgeführt. Bei der Perforation tritt arterielles Blut in den Subarachnoidalraum über. Das Perforationsmodell kommt der realen Situation der Aneurysmaruptur sehr nahe und eignet sich sehr gut als Modell für die Erforschung der primären Hirnschädigung (early brain injury) im Rahmen einer SAB. Bei diesem Modell lässt sich allerdings die Menge des austretenden Blutes nicht quantifizieren.

Dies kann bei bestimmten Fragestellungen wie der Erforschung von Risiken einer schwerwiegenden SAB von Nachteil sein. Des Weiteren wurde bei diesem Modell eine deutliche Variation in der Mortalitätsrate zwischen 15-50 \% beschrieben (Sehba 2014, Prunell et al. 2003, Sugawara et al. 2008). Eine verlässliche Induktion der Blutung mit unterschiedlichem Schweregrad und einer vertretbaren Mortalitätsrate ist wichtig, damit man 
die Anzahl der zu operierenden Tiere niedrig halten und verschiedene wissenschaftliche Fragestellungen beantworten kann. Damit eine SAB mit unterschiedlichem Schweregrad mit dem Perforationsmodell induziert werden kann, wurde versucht, für die Perforation unterschiedlich große Filamente zu benutzen. Es konnte ein stärkerer Anstieg des intrakraniellen Druckes im Falle einer SAB durch ein größeres Filament als Ausdruck der Schwere der SAB dokumentiert werden (Westermeier et al. 2009). Die Größe des Filaments

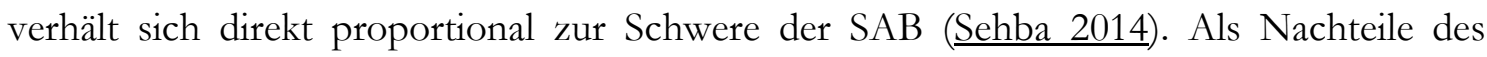
Perforationsmodells werden die große Variabilität sowohl in der Schwere der Blutung als auch in der Mortalität angesehen. Der wichtigste Vorteil ist die Möglichkeit zur Untersuchung der akuten Veränderungen nach der Ruptur eines arteriellen intrakraniellen Gefäßes (ehba 2014).

Beim Injektionsmodell wird eine definierte Blutmenge in den Subarachnoidalraum über verschiedene Zugangswege injiziert. Hierbei werden die einfachen und doppelten Injektionsmodelle unterschieden. Mit diesem Modell kann eine schwerwiegende SAB kontrolliert mit einer definierten Blutmenge induziert werden. In der Literatur wurden Blutmengen zwischen 0,15 $\mathrm{ml}$ und 0,3 $\mathrm{ml}$ beschrieben (Duhani et al. 2013, Vatter et al. 2006, Güresir et al. 2015a, Gules et al. 2002). Je schwerwiegender die SAB ist, desto höher ist allerdings auch die Mortalitätsrate. Damit eine schwerwiegende SAB induziert werden kann und gleichzeitig die Mortalitätsrate in einem ethisch vertretbaren Bereich bleibt, wurde in einem Modell, welches auch in dieser Studie zur Anwendung gekommen ist, die Blutmenge an zwei aufeinander folgenden Tagen injiziert (Lee et al. 2008, Güresir et al. 2015a). Als weitere Modifikation wurden Injektionen mit einem Abstand von zwei Tagen beschrieben (Lee et al. 2008). Als Vergleichsgruppen dienten 0,3 $\mathrm{ml}$ Blut versus 0,2 $\mathrm{ml}$ bei der ersten Injektion gefolgt von $0,2 \mathrm{ml}$ beziehungsweise $0,1 \mathrm{ml}$ bei der zweiten Injektion. Die Mortalität in der ersten Gruppe lag bei $40 \%$ verglichen mit 1,5\% in der zweiten Gruppe (Lee et al. 2008). Beim doppelten Injektionsmodell von Güresir et al. wird hingegen die gleiche Blutmenge von $0,25 \mathrm{ml}$ an zwei aufeinander folgenden Tagen injiziert. In diesem Fall lag die Mortalitätsrate bei $50 \%$ (Güresir et al. 2015a).

Bei tierexperimentellen Untersuchungen mit Kleintieren ist es schwierig, spät auftretende Komplikationen nach einer SAB im Versuchsmodell zu untersuchen. Dies beruht auf der Tatsache, dass die Versuchstiere im Vergleich zum Menschen einen schnelleren Metabolismus haben und dadurch das Blut aus dem Subarachnoidalraum schneller (meistens innerhalb der ersten 48 Stunden) eliminiert wird (Delgado et al. 1985, Gules et al. 2002).

Weiterhin wurden in der Literatur Unterschiede in der Blutverteilung unter den einzelnen SAB-Modellen festgestellt (Gules et al. 2002). Beim Perforationsmodell wurde die größte Blutmenge im Bereich der Perforation und an der Hirnoberfläche gesehen (Gules et al. 2002). Beim doppelten Injektionsmodell wird eine gleichmäßige Blutverteilung innerhalb der basalen Cisternen beschrieben, sodass alle großen basalen Hirnarterien von Blut umgeben sind (Güresir et al. 2015a). Bei den Injektionsmodellen der Ratte lässt sich ein zweizeitiges 
(frühe und späte Vasospasmen) Auftreten von Vasospasmen nachweisen, wie es auch beim Menschen der Fall ist (Gules et al. 2002). Bei einer Vergleichsstudie zwischen dem Perforationsmodell, dem einfachen Injektionsmodell (single hemorrhage model) und dem doppelten Injektionsmodell (double hemorrhage mode) konnte eine unterschiedliche Inzidenz zerebraler Vasospasmen gefunden werden. Die niedrigste Inzidenz wurde beim Perforationsmodell (16,5 \%) diagnostiziert, gefolgt vom einfachen Injektionsmodell $(20,1$ $\%$. Die höchste Inzidenz wurde beim doppelten Injektionsmodell (33,3 \%) beschrieben (Gules et al. 2002).

Im Injektionsmodell konnten Vasospasmen mit einem Maximum am Tag 5 in der Arteria basilaris detektiert werden (Güresir et al. 2010, Güresir et al. 2015b, Vatter et al. 2006). Weiterhin ist das doppelte Injektionsmodell durch die zweizeitige Blutinjektion dazu geeignet, spätere Komplikationen nach einer SAB, wie die verzögerte zerebrale Ischämie, zu untersuchen. In dieser Arbeit konnten wir anhand der histologischen Untersuchungen nach transkardialer Perfusion der Tiere am Tag 5 einen Großteil schwerwiegender Blutungen nachweisen.

$\mathrm{Ob}$ eine verzögerte Ischämie nach SAB-Induktion eintritt, ist von dem jeweiligen Kollateralkreislauf abhängig. Es konnte gezeigt werden, dass Ratten einen sehr guten Kollateralkreislauf des Gehirns aufweisen. Folglich ist es bei den Rattenmodellen schwierig, Vasospasmus-bedingte Infarkte zu induzieren. Eine einseitige Ligatur der Arteria carotis communis führt zu einer Reduktion der Durchblutung beider Hemisphären des Gehirns gleichermaßen, wie in einer experimentellen Studie nachgewiesen werden konnte (De Ley et al. 1985).

Folgende Gründe haben unsere Entscheidung für das doppelte Injektionsmodell bei dieser Arbeit beeinflusst: 1. Das Modell ist weit verbreitet, 2. Das Modell erlaubt eine kontrollierte Blutungsinduktion mit einer definierten Blutmenge, 3. Das Modell wird zur Untersuchung der verzögerten zerebralen Ischämie eingesetzt, wobei die Schwere der Blutung eine entscheidende Rolle spielt. Die Ergebnisse unserer Arbeit bestätigen die Hypothese früherer Arbeiten, dass dieses Modell insbesondere für die Untersuchung späterer Folgen nach einer SAB geeignet ist. Die Untersuchung sekundärer Hirnschäden wie der verzögerten zerebralen Ischämie, ist von besonderer Bedeutung, weil man diese Folgen potentiell durch Einleitung entsprechender prophylaktischer, diagnostischer und therapeutischer Maßnahmen in bestimmten Fällen verhindern kann.

\subsection{Diskussion der Klassifikationssysteme der Blutung}

Während verschiedene klinische Klassifikationen der SAB bereits definiert wurden, existieren keine etablierten Grading-Systeme für die Beschreibung der Schweregrade einer experimentellen SAB. Das liegt zum Teil daran, dass es viele tierexperimentelle SAB-Modelle gibt und existierende klinische Klassifikationen nicht direkt auf Tiermodelle übertragbar sind. 
Es gibt verschiedene Möglichkeiten, den Schweregrad einer SAB zu klassifizieren. Der klinisch-neurologische Zustand des Patienten zum Zeitpunkt des Blutungsereignisses wird als Grundlage vieler SAB-Klassifikationen beim Menschen verwendet. Zu diesen Klassifikationen zählen die Hunt- und Hess-Skala, die GCS und die WFNS-Skala. Alle diese Klassifikationen korrelieren mit der Prognose der Patienten und mit der Lebensqualität nach einer SAB (Kapapa et al. 2013). Die GCS zeigte dabei die beste Korrelation mit der Prognose nach einer SAB ( $\underline{\text { St Julien et al. 2008)}}$. Die WFNS-Skala basiert auf der GCS und wurde als Konsens der Weltgesellschaft der Neurochirurgen erstellt (Stalhammar und Starmark 1989). Außerdem wurde bei der Anwendung der GCS diese als am wenigsten Untersucher-abhängig definiert (Degen et al. 2011).

Weiterhin wird die Blutmenge, die zum Zeitpunkt der Aneurysmaruptur in den Subarachnoidalraum ausgetreten ist, als wichtiger Surrogatparameter für den Schweregrad der Blutung betrachtet und stellt die Basis vieler Klassifikationen dar. Das initiale Blutvolumen ist ein Prädiktor sowohl für die Morbidität als auch Mortalität infolge der SAB (Liu et al. 2016).

Das Vorhandensein einer intraventrikulären Blutung (Verteilung des Blutes zusätzlich innerhalb der Hirnventrikel) stellt ebenfalls für sich einen Risikofaktor zerebraler Vasospasmen und konsekutiver schlechter Prognose dar. Die Computertomographie, die zur Diagnosestellung geführt hat, ist die Grundlage für die Beurteilung der Blutmenge und der Blutverteilung. Die Beurteilung der Blutmenge kann qualitativ oder quantitativ erfolgen. Die quantitative Messung des Blutvolumens ist meistens aufwendig und in der klinischen Praxis nicht gut umsetzbar. Daher wurde bei den meisten Scores eine semiquantitative Einschätzung der Blutmenge verwendet. Des Weiteren wurde bei den verschiedenen Scores die Blutverteilung in unterschiedlichem Ausmaß berücksichtigt. Einige Scores haben den Fokus auf die Blutverteilung innerhalb der basalen Zisternen (Hijdra-Score), andere innerhalb der Hirnventrikel (Claassen-Score) und weitere werden unter der Berücksichtigung beider Aspekte (Fisher-Score) definiert (Fisher et al. 1980, Claassen et al. 2001, Hijdra et al. $\underline{1990)}$.

Der Hijdra-Score beurteilt die Blutverteilung innerhalb der basalen Cisternen und Fissuren (Sylvische Fissur, supraselläre Fissur, Cisterna ambiens und die Cisterna quadrigemina) sowie innerhalb der Hirnventrikel (linker und rechter Seitenventrikel, dritter Ventrikel und vierter Ventrikel). Die Blutmenge bei diesem Score wurde in 4 Kategorien unterteilt (kein Blut $=0$, kleine Blutmenge $=1$, moderate Blutmenge $=2$, vollständig mit Blut gefüllt $=3$ ). Es werden für jede Zisterne/Fissur/Ventrikel Punkte vergeben und die Summe als Score berechnet. Dieser Score hatte sich allerdings in der klinischen Praxis wenig durchgesetzt, wahrscheinlich wegen der doch aufwendigen Berechnung.

Im Jahr 1980 wurde der Fisher-Score zur Einteilung der SAB definiert. Der Fisher-Score basiert ebenfalls auf einer Beurteilung der Blutmenge, die auf der initialen CCTUntersuchung sichtbar ist. Der Fisher-Score wurde über die Jahre mehrfach modifiziert mit 
dem Ziel, die Blutverteilung innerhalb der Hirnventrikel, welche eine wichtige Rolle für die Einschätzung der Prognose spielt, zu berücksichtigen (Fisher et al. 1980, Frontera et al. 2006, Smith et al. 2005). Sowohl der Claassen-Score als auch der Graeb-Score setzen ihren Fokus auf die intraventrikuläre Blutausdehnung (Morgan et al. 2013, Claassen et al. 2001), was eine Messung des Blutvolumens in den Hirnventrikeln voraussetzt. Aus diesem Grund werden diese Scores häufiger zu wissenschaftlichen Zwecken verwendet, weil sie eine präzise Einteilung der Blutungsausdehnung ermöglichen. In der klinischen Praxis kommen sie aber wiederum aufgrund des Aufwandes selten zur Anwendung.

Die einzelnen Klassifikationen der SAB werden mit der Prognose der Erkrankung sowie mit dem Risiko für verschiedene Komplikationen korreliert (Friedman et al. 2002). Die Einschätzung des Risikos für mögliche Komplikationen nach einer SAB ist essentiell, damit diagnostische und therapeutische Maßnahmen rechtzeitig begonnen werden können. Die Blutmenge im Subarachnoidalraum ist einer der beständigsten Prädiktoren für das Auftreten

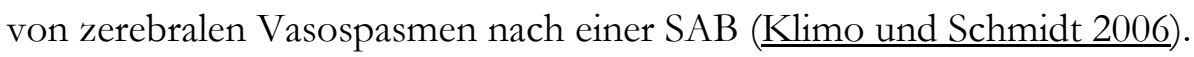

Die Klassifikation des Schweregrades einer experimentellen SAB bei Nagetieren kann parallel zu der SAB bei Menschen anhand der Klinik oder anhand von bildgebenden Untersuchungen oder post mortem mit Darstellung des Blutes innerhalb des Subarachoidalraumes und anhand von histologischen Untersuchungen erfolgen.

Die Schwere der SAB kann ebenfalls mittels Überwachung des intrakraniellen Druckes während der Induktion einer SAB bewertet werden. Je stärker der Anstieg des intrakraniellen Druckes, desto schwerwiegender ist die SAB (Sehba et al. 2011). Ein weiterer Ausdruck der Schwere der SAB ist die Normalisierung des zerebralen Blutflusses eine Stunde nach Induktion der SAB. Je schwerer die Blutung ist, desto stärker ist der Abfall des zerebralen Blutflusses nach Induktion der SAB und desto schwieriger erholt sich der zerebrale Blutfluss wieder. Wenn sich der zerebrale Blutfluss um weniger als $40 \%$ erholt, ist die Wahrscheinlichkeit hoch, dass die Tiere das Blutungsereignis nicht überleben ( 2011).

Nachdem sich der Fisher-Score mit seiner einfachen und verlässlichen Berechnung in der klinischen Praxis am besten durchgesetzt hat und eine hohe Aussagekraft bezüglich der Morbidität und Mortalität nach einer SAB aufweist, hatten wir bei dieser Arbeit diesen Score als Vorlage für die Definierung des Grading-Systems verwendet. Wir konnten die vier FisherGrade bei der experimentellen SAB reproduzieren. Um zu evaluieren, ob dieser Score auch bei anderen SAB-Modellen eingesetzt werden kann, sollte dies in zukünftigen Studien überprüft werden.

\subsection{Diskussion der histologischen Untersuchungen}

Die Gehirnentnahme und die nachfolgende histologische Aufarbeitung sind ein wichtiger Bestandteil der experimentellen SAB-Forschung. In dieser Arbeit haben wir die HE-Färbung 
für die Darstellung der Blutung verwendet. Hämosiderinablagerungen innerhalb des Subarachnoidalraumes sowie teilweise innerhalb der Hirnventrikel konnten visualisiert werden. Zusätzlich waren in diesem Bereich Makrophagen (Siderophagen) zu sehen. Siderophagen sind Zellen, welche für die Eliminierung des Blutes aus dem Subarachnoidalraum verantwortlich sind.

Mit der HE-Färbung lassen sich einzelne Erythrozyten sowie größere Blutansammlungen darstellen. Ebenfalls lässt sich durch eine Eisenreaktion das Alter der Blutung abschätzen sowie die Reaktion der Meningen auf die Blutung evaluieren.

Der Vorteil eines auf histologischen Untersuchungen basierenden Grading-Systems ist die Verfügbarkeit sowie die relativ einfache Durchführung. Ein Nachteil ist der Zeitpunkt der Untersuchung. Die Post-mortem-Analyse kann den initialen Schweregrad der Blutung potentiell beeinflussen. Dadurch kann nicht ausgeschlossen werden, dass der Schweregrad der initialen Blutung unterschätzt wird, da die Resorptionsraten des Blutes unter den einzelnen Versuchstieren einer Studie divergieren können. Aus diesem Grund ist es wichtig, zumindest beeinflussbare Faktoren wie Körpergewicht und Alter vergleichbar zu halten. Durch die Gehirnentnahme und Auswertung am Tag 5 nach Induktion der SAB kann nicht ausgeschlossen werden, dass kleinere Blutungen nicht mehr nachgewiesen werden können. Das kann potentiell das Vorliegen von Grad-1-Blutungen ohne histologisch sichtbare Blutung, wie bei zwei Fällen in der SAB-Gruppe in dieser Arbeit, erklären.

\subsection{Diskussion der MRT-Ergebnisse}

Die Magnetresonanztomographie (MRT) kommt regelmäßig in der tierexperimentellen SABForschung zum Einsatz (Guo et al. 2017, Güresir et al. 2012, Güresir et al. 2015b, Van den Bergh et al. 2005). Ziel ist beispielsweise die Darstellung der Blutung sowie die Evaluation von Vasospasmen oder Infarkten. Für diese Zwecke wird in der klinischen Praxis aus mehreren Gründen die CCT bevorzugt. Die CCT ist schneller durchführbar und insgesamt besser verfügbar als die MRT-Untersuchung. Des Weiteren können die Patienten während der Durchführung einer CCT besser überwacht werden als während der MRTUntersuchung. Patienten mit einer SAB benötigen oft ein invasives Monitoring über eine implantierte Sonde zur Messung des intrakraniellen Druckes oder der Hirndurchblutung. Diese invasiven Monitoringverfahren sind in der Regel nicht mit einer MRT-Untersuchung kompatibel. Auf der anderen Seite ist die CCT in der tierexperimentellen SAB-Forschung insbesondere mit kleinen Tieren wie Ratten aufgrund der schlechten Auflösung nicht geeignet.

Für die Darstellung der zerebralen Gefäße ist die digitale Subtraktionsangiographie (DSA) die Methode der Wahl. Obwohl diese Methode invasiv und aufwendig ist, wurde sie in der vaskulären tierexperimentellen Forschung regelmäßig eingesetzt. Bei der tierexperimentellen Forschung mit Kleintieren wie Ratten stellt dies aufgrund des kleinen Durchmessers der zerebralen Gefäße eine besondere Herausforderung dar. Aus diesem Grund hat sich die 
Mehrheit publizierter Studien auf die Darstellung der Arteria basilaris beschränkt. Einerseits aufgrund der guten Zugänglichkeit mit einem Katheter, und andererseits ist die Arteria basilaris eine der größten basalen zerebralen Arterien (Vatter et al. 2006, Van den Bergh et al. 2005).

Es wurden bisher einige Grading-Systeme für die Einteilung des Schweregrades einer SAB anhand der T2-Sequenzen bei Nagetieren vorgestellt. Diese Grading-Systeme erlauben eine in vivo Klassifikation der experimentellen SAB (Shishido et al. 2015, Egashira et al. 2015, Mutoh et al. 2016). Dieses Grading-System hatte eine signifikante Korrelation mit der vorhandenen Blutung im Subarachnoidalraum nach der Euthanasie der Tiere und direkter

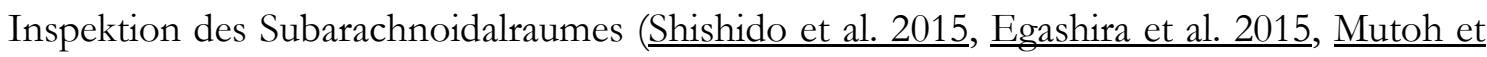
al. 2016).

Im Rahmen dieser experimentellen Studie konnte die Blutungsausdehnung im Subarachnoidalraum sowie in den Hirnventrikel anhand der MRT-Untersuchungen nur unzureichend dargestellt werden, so dass keine verlässliche Beurteilung der einzelnen SABSchweregrade durch die MRT-Untersuchungen möglich gewesen ist.

\subsection{Limitation und Stärke der Arbeit}

Eine der Limitationen dieser Arbeit ist die geringe Anzahl an Tieren, welche in die Auswertung eingeschlossen werden konnten. Ein weiterer Nachteil ist, dass die Klassifikation der SAB-Schwere erst post mortem durchgeführt wurde und nicht in vivo erfolgen konnte. Bei länger andauernden Experimenten kann dies von Nachteil sein, da möglicherweise kein histologischer Blutungsnachweis mehr möglich ist.

Stärke der Arbeit ist, dass eine verlässliche Reproduktion der vier Schweregrade analog der Fisher-Skala möglich gewesen ist. Somit können die Ergebnisse unter Anwendung dieses Grading-Systems einfacher auf die klinische Praxis übertragen werden, da die Fisher-Skala eine bereits etablierte und häufig eingesetzte Skala im klinischen Alltag sowie in klinischen Studien darstellt.

\subsection{Schlussfolgerung}

Das doppelte Blutinjektionsmodell der experimentellen Subarachnoidalblutung ermöglicht die Induktion einer hochgradigen SAB. Infolgedessen eignet sich dieses Modell insbesondere für die Untersuchung von schwerwiegenden SABs. Dieses Grading-System sollte bei anderen Tiermodellen der experimentellen Subarachnoidalblutung ebenfalls validiert werden, um einheitliche Standards in der experimentellen SAB-Forschung zu ermöglichen. 


\section{$5 \quad$ Zusammenfassung}

Die Einteilung der Schwere der SAB ist im klinischen Alltag von besonderer Bedeutung, weil die Klassifikationen der Blutung mit der Prognose der Patienten korrelieren. Bisher wurden viele dieser klinischen Klassifikationen zur Beurteilung des Schweregrades einer SAB beschrieben. Die Fisher-Skala ist in dieser Hinsicht die meist angewandte Skala im klinischen Alltag. Diese korreliert mit der Morbidität und Mortalität im Verlauf nach einer SAB. Im Gegensatz dazu gibt es bei der experimentellen SAB bisher kein etabliertes Grading-System für die Schwere der Blutung. Das Ziel dieser Arbeit war es, ein solches Grading-System analog der Fisher-Skala anhand des doppelten Injektionsmodells bei der Ratte zu definieren. Wir haben eine experimentelle SAB bei männlichen Sprague-Dawley-Ratten mit Hilfe des doppelten Injektionsmodells induziert. Zu diesem Zweck haben wir an zwei aufeinander folgenden Tagen 0,25 $\mathrm{ml}$ autologes arterielles Blut in die Cisterna magna injiziert. In der Sham-Gruppe wurde stattdessen die gleiche Menge Kochsalzlösung injiziert. Am Tag 5 nach Induktion der SAB wurde eine transkardiale Perfusion durchgeführt und das Gehirn der Versuchstiere zur histologischen Untersuchung entnommen. Nach Anfertigung einer HEFärbung wurde die Blutmenge und die Blutverteilung anhand der histologischen Schnitte analysiert.

Insgesamt wurden 52 Ratten in die Auswertung dieser Arbeit eingeschlossen, 47 Ratten waren in der SAB-Gruppe und 5 Ratten in der Sham-Gruppe. Es konnten folgende SABSchweregrade definiert werden: Grad 1 = keine Blutung, Grad 2 = lokal oder diffus verteilte geringe Blutmenge, Grad 3 = lokal oder diffus verteile große Blutmenge, Grad $4=$ intraventrikuläre Blutung. In der Sham-Gruppe konnte keine Blutung festgestellt werden (Grad 1). Eine Grad-1-Blutung wurde in 4,3\% (2/47), eine Grad-2-Blutung in 34\% (16/47), eine Grad-3-Blutung in 23,4 \% (11/47) und eine Grad-4-Blutung in 38,3\% (18/47) der Ratten in der SAB-Gruppe festgestellt. Die Fisher-Skala lässt sich anhand der histologischen Untersuchung im Rahmen des doppelten Injektionsmodells der Ratte verlässlich reproduzieren. Wir konnten mit dem doppelten Injektionsmodell in $62 \%(29 / 47)$ der Fälle eine schwerwiegende $\mathrm{SAB}$ induzieren. Die Ergebnisse unserer Arbeit unterstützen die Annahme, dass das doppelte Blutinjektionsmodell zur Untersuchung von späten Komplikationen wie der verzögerten zerebralen Ischämie besonderes geeignet ist. 


\section{$6 \quad$ Literaturverzeichnis}

Barry KJ, Gogjian MA, Stein BM (1979): Small animal model for investigation of subarachnoid hemorrhage and cerebral vasospasm. Stroke 10, 538-541

Bederson JB, Germano IM, Guarino L (1995): Cortical blood flow and cerebral perfusion pressure in a new noncraniotomy model of subarachnoid hemorrhage in the rat. Stroke 26, $1086-1091$

Budohoski KP, Guilfoyle M, Helmy A, Huuskonen T, Czosnyka M, Kirollos R, Menon DK, Pickard JD, Kirkpatrick PJ (2014): The pathophysiology and treatment of delayed cerebral ischemia following subarachnoid haemorrhage. J Neurol Neurosurg Psychiatry $\underline{85}, 1343-1353$

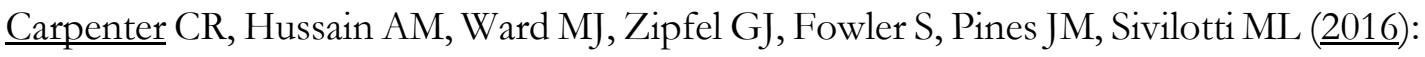
Spontaneous subarachnoid hemorrhage: a systematic review and meta-analysis describing the diagnostic accuracy of history, physical examination, imaging and lumber puncture with an exploration of test thresholds. Acad Emerg Med 23(9), 963-1003

Carrera E, Schmidt JM, Oddo M, Fernandez L, Claassen J, Seder D, Lee K, Badjatia N, Connolly ES, Mayer SA (2009): Transcranial Doppler for predicting delayed cerebral ischemia after subarachnoid hemorrhage. Neurosurgery 65(2), 316-323

Cavanagh SJ, Gordon VL (2002): Grading scales used in the management of aneurysmal subarachnoid hemorrhage: a critical review. J Neurosci Nurs 34(6), 288-295

Ciurea AV, Palade C, Voinescu D, Nica DA (013): Subarachnoid hemorrhage and cerebral vasospasm - literature review. J Med Life $\underline{6}(2), 120-125$

Claassen J, Bernardini GL, Kreiter K, Bates J, Du YE, Copeland D, Connolly S, Mayer SA (2001): Effect of cisternal and ventricular blood on risk of delayed cerebral ischemia after subarachnoid hemorrhage: The Fisher Scale revisited. Stroke 32, 2012-2020

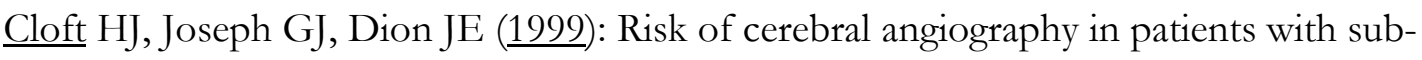
arachnoid hemorrhage, cerebral aneurysm, and arteriovenous malformation, a meta-analysis. Stroke $\underline{30}, 317-320$

Connolly ES, Rabinstein AA, Carhuapoma JR, Derdeyn CP, Dion J, Higashida RT, Hoh BL, Kirkness CJ, Naidech AM, Ogilvy CS (2012): Guidelines for the management of aneurysmal subarachnoid hemorrhage: a guideline for healthcare professionals from the American Heart Association/American Stroke Association. Stroke 43, 1711-1737

De Ley G, Nshimyumuremyi JB, Leusen I (1985): Hemispheric blood flow in the rat after unilateral common carotid occlusion: evolution with time. Stroke $\underline{16}$, 69-73 
De Oliveira Manoel AL, Mansur A, Murphy A, Turkel-Parrella D, Macdonald M, Macdonald RL, Montanera W, Marotta TR, Bharatha A, Effendi K (2014): Aneurysmal subarachnoid haemorrhage from a neuroimaging perspective. Crit Care $\underline{18}, 557$

De Oliveira Manoel AL, Jaja BN, Germans MR, Yan H, Qian W, Kouzmina E, Marotta TR, Turkel-Parrella D, Schweizer TA, Macdonald RL (2015): The vasograde: a simple grading scale for prediction of delayed cerebral ischemia after subarachnoid hemorrhage. Stroke $\underline{46}, 1826-1831$

De Rooij NK, Greving JP, Rinkel GJE, Frijns CJM (릭): Early prediction of delayed cerebral ischemia after subarachnoid hemorrhage: development and validation of a practical risk chart. Stroke 44, 1288-1294

Degen LA, Durhout Mees SM, Algra A, Rinkel GJ (2011): Interobserver variability of grading scales for aneurysmal subarachnoid hemorrhage. Stroke $\underline{42}$, 1546-1549

Delgado TJ, Brismar J, Svendgaard NA (1985): Subarachnoid haemorrhage in the rat: angiography and fluorescence microscopy of the major cerebral arteries. Stroke 16(4), 595-601

DGN-Leitlinie 2012 zur Subarachnoidalblutung. www.dgn.org

Dreier JP, Fabricius M, Ayata C, Sakowitz OW, William Shuttleworth C, Dohmen C, Graf R, Vajcoczy P, Helbok R, Suzuki M et al. (2017): Recording, analysis, and interpretation of spreading depolarizations in neurointensive care: Review and recommendations of the COSBID research group. J Cereb Blood Flow Metab 37(5), 1595-1625

Dreier JP (2011): The role of spreading depression, spreading depolarization and spreading ischemia in neurological desease. Nat Med 17(4), 439-447

D’Souza S (2015): Aneurysmal subarachnoid hemorrhage. J Neurosurg Anesthesiol 27, $222-240$

Dudhani RV, Kyle M, Dedeo C, Riodan M, Deshaies EM (2013): A low mortality rat model to assess delayed cerebral vasospasm after experimental subarachnoid hemorrhage. J Vis Exp $\underline{71}, 1-7$

Dupont SA, Wijdicks EF, Manno EM, Lanzino G, Rabinstein AA (2009): Prediction of angiographic vasospasm after aneurysmal subarachnoid hemorrhage: value of the Hijdra sum scoring system. Neurocrit Care 11(2), 172-176

Edlow JA, Caplan LR (2000): Avoiding pitfalls in the diagnosis of subarachnoid Hemorrhage. N Engl J Med 342(1), 29-36

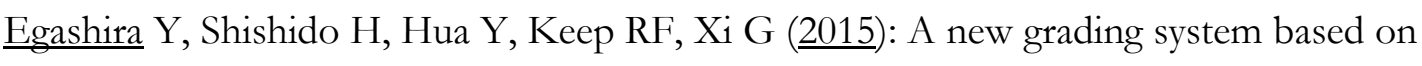
magnetic resonance imaging in a mouse model of subarachnoid hemorrhage. Stroke $\underline{46}$, $582-584$ 


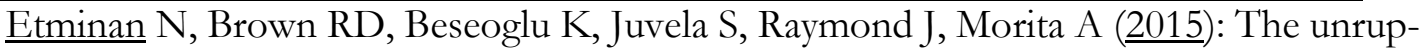
tured intracranial aneurysm treatment score: a multidisciplinary consensus. Neurology $\underline{85}(10), 881-889$

Feigin VL, Rinkel GJE, Lawes CMM, Algra A, Bennett DA, van Gijn J, Anderson CS (2005): Risk factors for subarachnoid hemorrhage: an updated systematic review of epidemiological studies. Stroke $\underline{36}, 2773-2780$

Feigin VL, Lawes CM, Bennett DA, Barker-Collo SL, Parag V (2009): Worldwide stroke incidence and early case fatality reported in 56 population-based studies: a systematic review. Lancet Neurol $\underline{8}(4), 355-369$

Fisher CM, Kistler JP, Davis JM (1980): Relation of cerebral vasospasm to subarachnoid hemorrhage visualized by computerized tomographic scanning. Neurosurgery $\underline{16}(1), 1-9$

Foreman B (2016): The pathophysiology of delayed cerebral ischemia. J Clin Neurophysiol $\underline{33}(3), 174-182$

Francoeur CL, Mayer SA (2016): Management of delayed cerebral ischemia after subarachnoid hemorrhage. Crit Care 20, 277

Friedman JA, Goerss SJ, Meyer FB, Piepgras DG, Pichelmann MA, Mclver JI, Toussaint LG 3rd, McClelland RL, Nichols DA, Atkinson JL (2002): Volumetric quantification of Fisher Grade 3 aneurysmal subarachnoid hemorrhage: a novel method to predict symp-

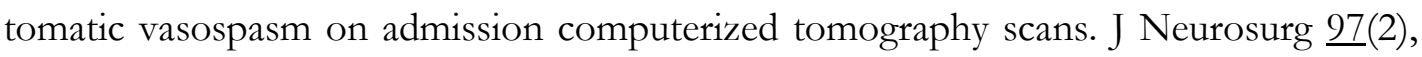
$401-407$

Frontera JA, Claasen J, Schmidt JM, Wartenberg KE, Temes R, Connolly ES Jr, MacDonald RL, Mayer SA (2006): Prediction of symptomatic vasospasm after subarachnoid hemorrhage: the modified fisher scale. Neurosurgery $\underline{59}(1), 21-27$

Frontera JA, Fernandez A, Schmidt JM, Claassen J, Wartenberg KE, Badjatia N, Connolly S, Mayer SA (2009): Defining vasospasm after subarachnoid hemorrhage: What is the most clinically relevant definition? Stroke $\underline{40}, 1963-1968$

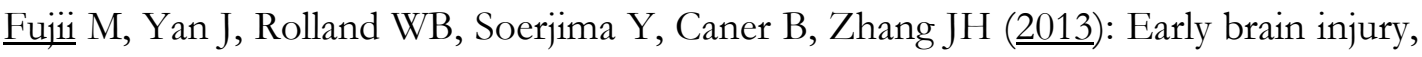
an evolving frontier in subarachnoid hemorrhage research. Trans Stroke Res 14(4), 432446

Greving JP, Wermer MJH, Brown RD, Morita A, Juvela S, Yonekura M (2014): Development of the PHASES score for prediction of risk of rupture of intracranial aneurysms: a pooled analysis of six prospective cohort studies. Lancet Neurology 13(1), 59-66

Gules I, Satoh M, Clower BR, Nanda A, Zhang JH (2002): Comparison of three rat models of cerebral vasospasm. Am J Physiol Heart Circ Physiol 283, 2551-2559 
Guo D, Wilkinson DA, Thompson BG, Pandey AS, Keep RF, Xi G, Hua Y (2017): MRI characterization in the acute phase of experimental subarachnoid hemorrhage. Transl Stroke Res $\underline{8}(3), 234-243$

Güresir E, Raabe A, Jiimsin A, Dias S, Raab P, Seifert V, Vatter H (2010): Histological evidence of delayed ischemic brain tissue damage in the rat double-hemorrhage model. J Neurol Sci 293(1-2), 18-22

Güresir E, Vasiliadis N, Dias S, Raab P, Seifert V, Vatter H (2012): The effect of common carotis artery occlusion on delayed brain tissue damage in the rat double subarachnoid hemorrhage model. Acta Neurochir $\underline{154}, 11-19$

Güresir E, Schuss P, Borger V, Vatter H (2015a): Rat cisterna magna double-injection model of subarachnoid hemorrhage - background, advantages/limitations, technical considerations, modifications, and outcome measures. Acta Neurochir Suppl 120, 325329

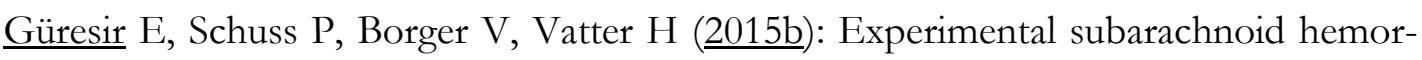
rhage: double cisterna magna injection rat model - assessment of delayed pathological effects of cerebral vasospasm. Transl Stroke Res $\underline{6}$ (3), 242-251

Helbok R, Schiefecker AJ, Beer R, Dietmann A, Antunes AP, Sohm F, Fischer M, Hackl WO, Rhomberg P, Lackner P (2015): Early brain injury after aneurysmal subarachnoid hemorrhage: a multimodal neuromonitoring study. Crit Care 19, 75

Hendrix P, Foreman PM, Harrigan MR, Fisher WS $3^{\text {rd }}$, Vyas NA, Lipsky RH, Lin M, Walters BC, Tubbs RS, Shoja MM (2017): Endothelial nitric oxide synthase polymorphism is associated with delayed cerebral ischemia following aneurysmal subarachnoid hemorrhage. World Neurosurg 101, 514-519

Hijdra A, Brouwers PJ, Vermeulen M, van Gijn J (1990): Grading the amount of blood on computed tomograms after subarachnoid hemorrhage. Stroke 21(8), 1156-1161

Hunt WE, Hess RM (1968): Surgical risk as related to time of intervention in the repair of intracranial aneurysms. J Neurosurg 28 (1), 14-20

Inagawa T, Yahara K, Ohbayashi N (2014): Risk factors associated with cerebral vasospasm following aneurysmal subarachnoid hemorrhage. Neurol Med Chir $\underline{54}$, 465-473

Jakobsson KE, Saveland H, Hillman J (1996): Warning leak and management outcome

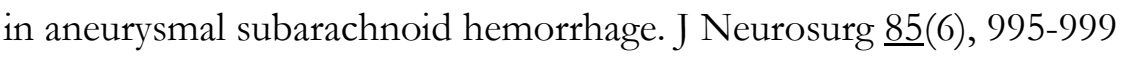

Jeon H, Ai J, Sabri M, Tariq A, Shang X, Chen G, Macdonald RL (2009): Neurological and neurobehavioral assessment of experimental subarachnoid hemorrhage. BMC Neurosci $\underline{10}, 103$

Juvela S, Poussa K, Porras M (2001): Factors affecting formation and growth of intracranial aneurysms: a long-term follow-up study. Stroke $\underline{32}$, 485-491 
Kapapa T, Tjahjadi M, König R, Wirtz CR, Woischneck D (2013): Which clinical variable influences health-related quality of life the most after spontaneous subarachnoid hemorrhage? Hunt and Hess scale, Fisher score, World Federation of Neurosurgeons score, Brussels coma score, and Glasgow coma score compared. World Neurosurg $\underline{80}(6), 853-$ 858

Kassell NF, Torner JC, Haley EC Jr, Jane JA, Adams HP, Kongable GL (1990a): The international cooperative study on the timing of aneurysm surgery. Part 1: Overall management results. J Neurosurg $\underline{73}(1), 18-36$

Kassell NF, Torner JC, Jane JA, Haley EC Jr, Adams HP (1990b): The international cooperative study on the timing of aneurysm surgery. Part 2: Surgical results. J Neurosurg $\underline{73}(1), 37-47$

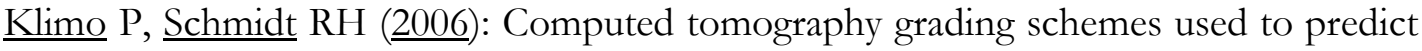
cerebral vasospasm after aneurysmal subarachnoid hemorrhage: a historical review. Neurosurg Focus 21(3), E5

Kramer AH, Mikolaenko I, Deis N, Dumont AS, Kassell NF, Bleck TP, Nathan BA (2010): Intraventricular hemorrhage volume predicts poor outcomes but not delayed ischemic neurological deficits among patients with ruptured cerebral aneurysms. Neuro-

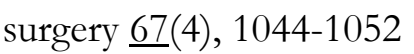

Latigua H, Ortega-Gutierrez S, Schmidt JM, Lee K, Badjatia N, Agarwal S, Claassen J, Connolly ES, Mayer SA (2015): Subarachnoid hemorrhage: who dies, and why? Crit Care $\underline{19}, 309$

Leber KA, Kurschel-Lackner S, Gellner V, Wießpeiner U (2008): Subarachnoidalblutung: Herausforderung einer Diagnose. J Neurol Neurochir Psychiatr $\underline{9}(1), 28-32$

Lee JY, Huang DL, Keep R, Sagher O (2008): Characterization of an improved double hemorrhage rat model for the study of delayed cerebral vasospasm. J Neurosci Methods $\underline{168}(2), 358-366$

Lindbohm JV, Kaprio J, Jousilahti P, Salomaa V, Korja M (2016): Sex, smoking and risk for subarachnoid hemorrhage. Stroke $\underline{47}, 1-7$

Liu J, Song J, Zhao D, Li H, Lu Y, Wu G, Hou K, Gao X (2016): Risk factors responsible for the volume of hemorrhage in aneurysmal subarachnoid hemorrhage. Neurology India 64(4), 686-691

Malinova V, Schatlo B, Voit M, Suntheim P, Rohde V, Mielke D (2016a): Identification of specific age groups with a high risk for developing cerebral vasospasm after aneurysmal subarachnoid hemorrhage. Neurosurg Rev $\underline{39}$ (3), 429-436 


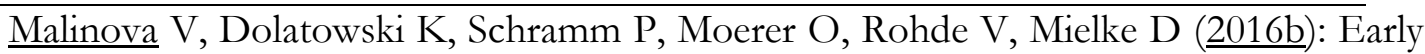
whole-brain CT perfusion for detection of patients at risk for delayed cerebral ischemia after subarachnoid hemorrhage. J Neurosurg 125(1), 128-136

Molyneux A, Kerr R, Stratton I, Sandercock P, Clarke M, Shrimptom J, Holman R (2002): International subarachnoid aneurysm trial (ISAT) of neurosurgical clipping versus endovascular coiling in 2143 patients with ruptured intracranial aneurysms: a randomized trial. Lancet $\underline{360}(9342), 1267-1274$

Molyneux AJ, Birks J, Clarke BA, Sneade BA, Kerr RSC (ㅁ15): The durability of endovascular coiling versus neurosurgical clipping of ruptured cerebral aneurysma: 18 years follow-up of the UK cohort of the international subarachnoid aneurysm trial (ISAT). Lancet $\underline{385}(9969)$, 691-697

Morgan TC, Dawson J, Sprengler D, Lees KR, Aldrich C, Mishra NK, Lane K, Quinn TJ, Diener-West M, Weir CJ (2013): The modified Graeb Score: an enhanced tool for intraventricular hemorrhage measurement and prediction of functional outcome. Stroke 44, 635-641

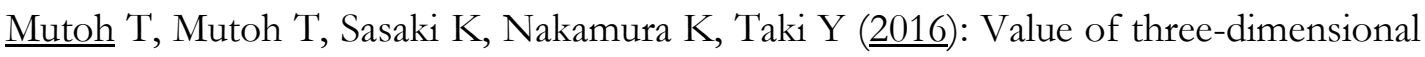
maximum intensity projection display to assist in magnetic resonance imaging (MRI)based grading in mouse model of subarachnoid hemorrhage. Med Sci Monit 22, 20502055

Naraoka M, Matsuda N, Shimamura N, Asano K, Ohkuma H (2014): The role of arterioles and the microcirculation in the development of vasospasm after aneurysmal SAH. Biomed Res Int 25, 37-46.

Prunell GF, Mathiesen T, Diemer NH, Svendgaard NA (2003): Experimental subarachnoid hemorrhage: subarachnoid blood volume, mortality rate, neuronal death, cerebral blood flow, and perfusion pressure in three different rat models. Neurosurgery $\underline{52(1)}$, 165-175.

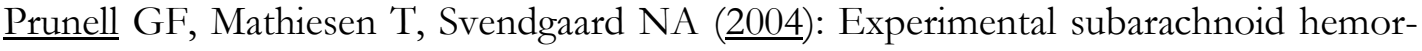
rhage: cerebral blood flow and brain metabolism during the acute phase in three different models in the rat. Neurosurgery $\underline{54}(2), 426-36$

Raslan F, Albert-Weißenberger C, Westermeier T, Saker S, Kleinschmitz C, Lee JY (2012): A modified double injection model of cisterna magna for the study of delayed cerebral vasospasm following subarachnoid hemorrhage in rats. Experimental \& Translational Stroke Medicine 4, 23

Raymond J, Guillemin F, Proust F, Molyneux AJ, Fox AJ, Claiborne JS, Meder JF, Rouleau I (2008): Unruptured intracranial aneurysms. A critical review of the international study of unruptured intracranial aneurysms (ISUIA) and of appropriate methods to address the clinical problem. Interv Neuroradiol 14(1), 85-96 


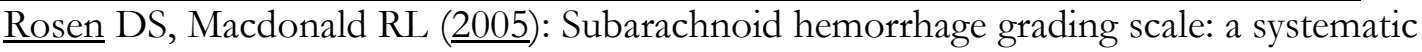
review. Neurocrit Care 2(2), 110-118

Rosengart AJ, Schultheiss KE, Tolentino J, Macdonald RL (2007): Prognostic factors for outcome in patients with aneurysmal subarachnoid hemorrhage. Stroke $\underline{38}$, 2315-2321

Rowland MJ, Hadjipavlou G, Kelly J, Westbrook J, Pattinson KTS (2012): Delayed cerebral ischemia after subarachnoid hemorrhage: looking beyond vasospasm. Br J of Anaesth 109(3):315-329

Schmieder K, Heuser L, Skodda S (2007): Vorgehen bei nicht traumatischer Subarachnoidalblutung des Erwachsenen. Dtsch Arztebl 104(39), 2649-2654

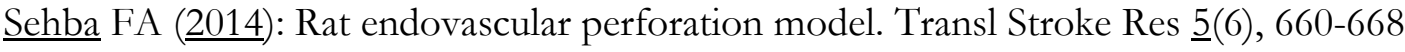

Sehba FA, Pluta RM, Zhang JH (2011): Metamorphosis of subarachnoid hemorrhage research: from delayed vasospasm to early brain injury. Mol Neurobiol $\underline{43}$, 27-40

Shishido H, Egashira Y, Okudo S, Zhang H, Hua Y, Keep RF, Xi G (2015): A magnetic resonance imaging grading system for subarachnoid hemorrhage severity in a rat model. J Neurosci Methods $\underline{243}, 115-119$

Smith ML, Abrahams JM, Chandela S, Smith MJ, Hurst RW, Le Roux PD (2005): Subarachnoid hemorrhage on computed tomography scanning and the development of cerebral vasospasm: the Fisher grade revisited. Surg Neurol 63(3), 229-234

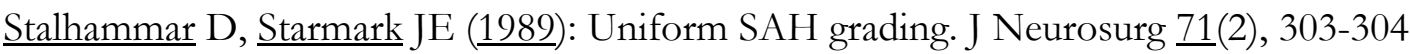

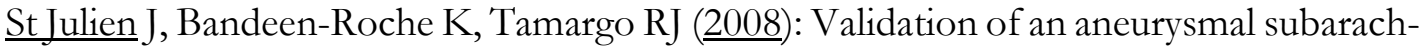
noid hemorrhage grading scale in 1532 consecutive patients. Neurosurgery $\underline{63}(2), 204-$ 210

Sugawara T, Ayer R, Jadhav V, Zhang JH (ㅁ0ㅇ): A new grading system evaluating bleeding scale in filament perforation subarachnoid hemorrhage rat model. J Neurosci Methods $\underline{167}(2), 327-334$

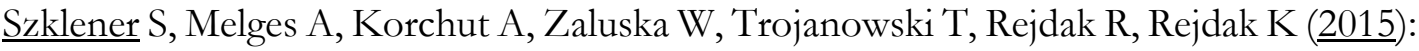
Predictive model for patients with poor-grade subarachnoid haemorrhage in 30-day observation: a 9-year cohort study. BMJ $\underline{5}(6)$, e007795.

Teasdale G, Jennett B (1972): Assessment of coma and impairment consciousness. A practical scale. Lancet $\underline{2}, 81-84$

Teasdale GM, Drake CG, Hunt W, Kassell N, Sano K, Pertuiset B, De Villiers JC (1988): A universal subarachnoid hemorrhage scale: report of a committee of the World Federation of Neurosurgical Societies. J Neurol Neurosurg Psychiatry $\underline{51}, 1457$

Teasdale GM, Wardlaw JM, White PM, Murray G, Teasdale EM, Easton V (2005): The familial risk of subarachnoid haemorrhage. Brain $\underline{128}, 1677-1685$ 
Vatter H, Weidauer S, Konczalla J, Dettmann E, Zimmermann M, Raabe A, Preibisch C, Zanella FE, Seifert V (2006): Time course in the development of cerebral vasospasm after experimental subarachnoid hemorrhage: clinical and neuroradiological assessment of the rat double hemorrhage model. Neurosurgery $\underline{58}, 1190-1197$

Van den Bergh WM, Schepers J, Veldhuis WB, Nicolay K, Tulleken CAF, Rinkel GJE (2005): Magnetic resonance imaging in experimental subarachnoid hemorrhage. Acta Neurochir 147, 977-983

Van Gijn J, Rinkel GJE (2001): Subarachnoid hemorrhage: diagnosis, causes and management. Brain 124, 249-278

Van Norden AG, van Dijk GW, van Hiuzen MD, Algra A, Rinkel GJ (2006): Interobserver agreement and predictive value for outcome of two rating scales for the amount of axtravasated blood after aneurysmal subarachnoid haemorrhage. J Neurol 253(9), $1217-1220$

Vergouwen MD, Vermeulen M, Coert BA, Stroes ES, Roos YB (2008): Microthrombosis after aneurysmal subarachnoid hemorrhage: an additional explanation for delayed cerebral ischemia. J Cereb Blood Flow Metab 28(11), 1761-1770

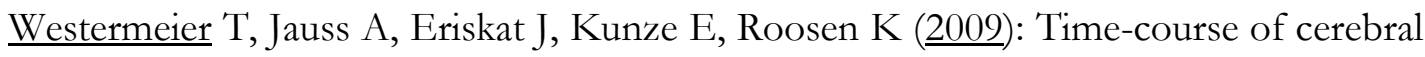
perfusion and tissue oxygenation in the first $6 \mathrm{~h}$ after experimental subarachnoid hemorrhage in rats. J Cereb Blood Flow Metab 29 (4), 771-779

Wiebers DO, Whisnant JP, Huston J3rd, Meissner I, Brown RD Jr, Piepgras DG, Forbes

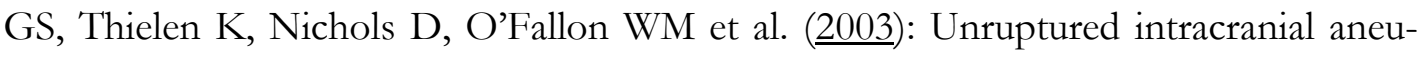
rysms: natural history, clinical outcome, and risks of surgical and endovascular treatment. Lancet $\underline{362}$ (9378), 103-10

Xia ZW, Liu XM, Wang JY, Cao H, Chen FH, Huang J, Li QZ, Fan SS, Jiang B, Chen ZG (2017): Coiling is not superior to clipping in patients with high-grade aneurysmal subarachnoid hemorrhage: systematic review and meta-analysis. World Neurosurg $\underline{98}$, $411-420$

Zheng Z, Sanchez-Porras R, Santos E, Unterberg AW, Sakowitz OW (2012): Delayed cerebral ischemia after subarachnoid hemorrhage: from vascular spasm to cortical spreading depolarization. Curr Neurovasc Res $\underline{9}$ (4), 310-319 


\section{Danksagung}

An dieser Stelle möchte ich mich bei meiner Betreuerin Frau Prof. Dr. Mielke für ihre Unterstützung während der Planung der Experimente sowie während der Auswertung der Daten und der Verfassung der Arbeit bedanken. Des Weiteren danke ich meinem CoBetreuer PD Dr. Psychogios für die Unterstützung bei der Datenauswertung.

Ein besonderer Dank geht auch an Prof. Dr. Rohde für die Möglichkeit, die Tierexperimente durchzuführen. Ebenfalls danke ich Frau Bleuel Kim und Herrn Bogdan Iliev für die gute Zusammenarbeit bei der Durchführung der Experimente und der Datengewinnung. 